quatrième série-tome $41 \quad$ fascicule $1 \quad$ janvier-février 2008

$$
\begin{aligned}
& \text { ANNALES } \\
& \text { SCIENTIFIQUES } \\
& \text { de } \\
& \text { L'ECOLE } \\
& \text { NORMALE } \\
& \text { SUPÉRIEURE }
\end{aligned}
$$

Jean-François COULOMBEL \& Paolo SECCHI

Nonlinear compressible vortex sheets in two space dimensions 
Ann. Scient. Éc. Norm. Sup.

$4^{\text {e }}$ série, t. 41,2008, p. 85 à 139

\title{
NONLINEAR COMPRESSIBLE VORTEX SHEETS IN TWO SPACE DIMENSIONS
}

\author{
By Jean-François COUlombel and Paolo SECCHi
}

\begin{abstract}
We consider supersonic compressible vortex sheets for the isentropic Euler equations of gas dynamics in two space dimensions. The problem is a free boundary nonlinear hyperbolic problem with two main difficulties: the free boundary is characteristic, and the so-called Lopatinskii condition holds only in a weak sense, which yields losses of derivatives. Nevertheless, we prove the local existence of such piecewise smooth solutions to the Euler equations. Since the a priori estimates for the linearized equations exhibit a loss of regularity, our existence result is proved by using a suitable modification of the Nash-Moser iteration scheme. We also show how a similar analysis yields the existence of weakly stable shock waves in isentropic gas dynamics, and the existence of weakly stable liquid/vapor phase transitions.
\end{abstract}

RÉSUMÉ. - Nous construisons des nappes de tourbillon supersoniques pour les équations d'Euler compressibles isentropiques en deux dimensions d'espace. Il s'agit d'un problème non-linéaire hyperbolique à frontière libre présentant deux difficultés principales : la frontière libre est caractéristique et la condition dite de Lopatinskii n'est satisfaite que dans un sens faible, ce qui induit des estimations à perte. Néanmoins nous montrons l'existence de telles solutions régulières par morceaux des équations d'Euler en utilisant un schéma itératif de type Nash-Moser palliant les pertes de régularité. Notre analyse s'étend au cas de discontinuités non-caractéristiques et faiblement stables comme certaines ondes de choc pour les équations d'Euler ou les transitions de phase liquide- vapeur.

\section{Introduction}

The Cauchy problem for the compressible Euler equations in several space dimensions is a major challenge in the domain of hyperbolic conservation laws. The (local in time) existence of smooth solutions away from vacuum follows from a general Theorem by Kato [20], while the existence of smooth solutions with vacuum is proved by Chemin in [8]. Due to the finite time blow-up of smooth solutions, see [38], it is natural to look for weak solutions. The construction of (local in time) piecewise smooth solutions is a preliminary step. The 
first breakthrough in this direction is the existence of one multidimensional uniformly stable shock wave, that was obtained by Majda [24, 23], see also [6] for a different approach. The existence of two uniformly stable shock waves was shown by Métivier [27]. Then the existence of multidimensional rarefaction waves was obtained by Alinhac [1]. More recently, Francheteau and Métivier [14] have studied the asymptotic behavior of multidimensional shock waves when the strength of the shock tends to zero. The limit of such weak shock waves are sonic waves, whose existence has been proved by Métivier [28]. All these works are based on an appropriate iterative scheme (either a standard Picard iteration or a Nash-Moser iteration), that is proved to converge thanks to a tame estimate on the linearized equations. In this work, we show the existence of contact discontinuities in two space dimensions for the isentropic Euler equations. A similar analysis could be done for the nonisentropic Euler equations, since the stability properties of contact discontinuities for the isentropic Euler equations, and for the nonisentropic Euler equations are quite similar ${ }^{(1)}$.

Let us recall briefly the important features of Majda's work on shock waves. The existence result [23] was obtained under a uniform stability assumption, that ensures a good a priori estimate for the linearized equations. By "good" a priori estimate, we mean an estimate where there is no loss of regularity from the source terms to the solution. However, this uniform stability condition is not satisfied by all shock waves in gas dynamics ${ }^{(2)}$. Furthermore, this uniform stability condition (or more precisely the analogue of this condition for characteristic discontinuities), is never satisfied by contact discontinuities in two or three space dimensions, see e.g. [30, 13] or [37, page 222]. As a matter of fact, in three space dimensions, every contact discontinuity is violently unstable (this violent instability is the analogue of the Kelvin-Helmholtz instability for incompressible fluids), while in two space dimensions, a large jump of the tangential velocity makes the contact discontinuity weakly stable. A precise study of this weak stability has been performed in [12], where we have shown that for such weakly stable contact discontinuities, the linearized equations satisfy an a priori estimate with a loss of one derivative. In this case, we cannot hope to prove the existence of solutions to the nonlinear problem by means of a Picard iteration. In this paper, we shall show that a suitable Nash-Moser iteration converges towards a contact discontinuity solution to the Euler equations.

At the end of the paper, we give two other situations where our analysis applies. More precisely, we can apply the same type of iteration scheme to show the existence of weakly stable shock waves in two or three space dimensions, and the existence of liquid/vapor phase transitions in two or three space dimensions. Roughly speaking, our work shows that the weak Lopatinskii condition, that is known to be sufficient for linear well-posedness [10], is also sufficient for nonlinear well-posedness (even when the verification of the weak Lopatinskii condition is submitted to nonlinear constraints). However, we prefer not to give the proof of such an abstract result, and we shall focus on the problem of contact discontinuities for the

(1) We refer the reader to [30, 13, 11, 32] for the stability criterion in the nonisentropic case.

(2) The stability of shock waves heavily depends on the pressure law, but the general idea is that shock waves of moderate strength are uniformly stable, while large shock waves may be only weakly stable.

$4^{\mathrm{e}}$ SÉRIE - TOME $41-2008-\mathrm{N}^{\mathrm{o}} 1$ 
Euler equations since it gathers the two main difficulties, namely a characteristic free boundary, and the weak Lopatinskii condition under nonlinear constraints. The main steps of the analysis are outlined so our method can be applied to various situations.

\section{The nonlinear equations}

We consider the isentropic Euler equations in the whole plane $\mathbb{R}^{2}$. Denoting by $\mathbf{u} \in \mathbb{R}^{2}$ the velocity of the fluid, and by $\rho$ its density, the equations read:

$$
\left\{\begin{array}{l}
\partial_{t} \rho+\nabla_{x} \cdot(\rho \mathbf{u})=0, \\
\partial_{t}(\rho \mathbf{u})+\nabla_{x} \cdot(\rho \mathbf{u} \otimes \mathbf{u})+\nabla_{x} p=0,
\end{array}\right.
$$

where $p=p(\rho)$ is the pressure law. In all this paper $p$ is a $\mathcal{C}^{\infty}$ function of $\rho$, defined on ] $0,+\infty\left[\right.$, and such that $p^{\prime}(\rho)>0$ for all $\rho$. The speed of sound $c(\rho)$ in the fluid is defined by the relation:

$$
\forall \rho>0, \quad c(\rho):=\sqrt{p^{\prime}(\rho)} .
$$

It is a well-known fact that, for such a pressure law, (1) is a strictly hyperbolic system in the region $] 0,+\infty\left[\times \mathbb{R}^{2}\right.$, and (1) is also symmetrizable.

In all what follows, the first and second coordinates of the velocity field are denoted respectively $v$, and $u$, that is, $\mathbf{u}=(v, u) \in \mathbb{R}^{2}$. Then, for all $\left.U=(\rho, \mathbf{u}) \in\right] 0,+\infty\left[\times \mathbb{R}^{2}\right.$, we define the following matrices:

$$
A_{1}(U):=\left(\begin{array}{ccc}
v & \rho & 0 \\
\frac{p^{\prime}(\rho)}{\rho} & v & 0 \\
0 & 0 & v
\end{array}\right), \quad A_{2}(U):=\left(\begin{array}{ccc}
u & 0 & \rho \\
0 & u & 0 \\
\frac{p^{\prime}(\rho)}{\rho} & 0 & u
\end{array}\right) .
$$

In the region where $(\rho, \mathbf{u})$ is smooth, (1) is equivalent to the quasilinear equations:

$$
\partial_{t} U+A_{1}(U) \partial_{x_{1}} U+A_{2}(U) \partial_{x_{2}} U=0 .
$$

In this paper, we are interested in solutions to (1) that are smooth on either side of a surface $\Gamma:=\left\{x_{2}=\varphi\left(t, x_{1}\right), t \in[0, T], x_{1} \in \mathbb{R}\right\}$, and such that, at each time $t \in[0, T]$, the tangential velocity is the only quantity that experiments a jump across the curve $\Gamma(t)$. (Tangential should be understood as tangential with respect to $\Gamma(t)$.) The density, and the normal velocity should be continuous across $\Gamma(t)$. For such solutions, the jump conditions across $\Gamma$ read:

$$
\partial_{t} \varphi=-v^{+} \partial_{x_{1}} \varphi+u^{+}=-v^{-} \partial_{x_{1}} \varphi+u^{-}, \quad \rho^{+}=\rho^{-} .
$$

As detailed in [12], for the Euler equations (1), these solutions are exactly the contact discontinuities in the sense of Lax [21]. Recall that the second characteristic field of (1) is linearly degenerate, and thus, gives rise to contact discontinuities. For such discontinuous solutions, there is no mass transfer from one side of $\Gamma(t)$ to the other. (Shock waves are exactly the opposite situation where there is a mass transfer from one side to the other.)

The discontinuity surface $\Gamma$ is part of the unknowns, and it is convenient to reformulate the problem in the fixed domain $\left\{t \in[0, T], x_{1} \in \mathbb{R}, x_{2} \geq 0\right\}$, by introducing a change of variables. This change of variables is detailed in [12, section 2], see also [1, 24, 29]. After 
fixing the unknown front, we are led to constructing smooth solutions $U^{ \pm}=\left(\rho^{ \pm}, v^{ \pm}, u^{ \pm}\right)$, $\Phi^{ \pm}$, to the following system of equations:

(3) $\partial_{t} U^{ \pm}+A_{1}\left(U^{ \pm}\right) \partial_{x_{1}} U^{ \pm}+\frac{1}{\partial_{x_{2}} \Phi^{ \pm}}\left(A_{2}\left(U^{ \pm}\right)-\partial_{t} \Phi^{ \pm}-\partial_{x_{1}} \Phi^{ \pm} A_{1}\left(U^{ \pm}\right)\right) \partial_{x_{2}} U^{ \pm}=0$,

in the interior domain $\left\{t \in[0, T], x_{1} \in \mathbb{R}, x_{2}>0\right\}$, with the boundary conditions:

$$
\begin{aligned}
& \Phi_{\left.\right|_{x_{2}=0}}^{+}=\Phi_{\left.\right|_{x_{2}=0}}^{-}=\varphi, \\
& \left(v^{+}-v^{-}\right)_{\left.\right|_{x_{2}=0}} \partial_{x_{1}} \varphi-\left(u^{+}-u^{-}\right)_{\left.\right|_{x_{2}=0}}=0 \text {, } \\
& \partial_{t} \varphi+v_{\left.\right|_{x_{2}}=0}^{+} \partial_{x_{1}} \varphi-u_{\left.\right|_{x_{2}=0}}^{+}=0, \\
& \left(\rho^{+}-\rho^{-}\right)_{\left.\right|_{x_{2}=0}}=0 \text {. }
\end{aligned}
$$

We will also consider the initial conditions:

$$
\left(\rho^{ \pm}, v^{ \pm}, u^{ \pm}\right)_{\mid t=0}=\left(\rho_{0}^{ \pm}, v_{0}^{ \pm}, u_{0}^{ \pm}\right)\left(x_{1}, x_{2}\right), \quad \varphi_{\mid t=0}=\varphi_{0}\left(x_{1}\right) .
$$

The functions $\Phi^{ \pm}$should satisfy the constraints:

$$
\forall\left(t, x_{1}, x_{2}\right) \in[0, T] \times \mathbb{R} \times \mathbb{R}^{+}, \quad \pm \partial_{x_{2}} \Phi^{ \pm}(t, x) \geq \kappa,
$$

for a suitable constant $\kappa>0$, as well as the eikonal equations:

$$
\partial_{t} \Phi^{+}+v^{+} \partial_{x_{1}} \Phi^{+}-u^{+}=\partial_{t} \Phi^{-}+v^{-} \partial_{x_{1}} \Phi^{-}-u^{-}=0
$$

in the whole domain $\left\{t \in[0, T], x_{1} \in \mathbb{R}, x_{2}>0\right\}$. Before going on, let us make a few remarks:

REMARK 1. - The constraint (6) ensures that the mapping:

$$
\left(t, x_{1}, x_{2}\right) \longmapsto \begin{cases}\left(t, x_{1}, \Phi^{+}\left(t, x_{1}, x_{2}\right)\right), & \text { if } x_{2}>0, \\ \left(t, x_{1}, \Phi^{-}\left(t, x_{1},-x_{2}\right)\right), & \text { if } x_{2}<0,\end{cases}
$$

is a change of variables that straightens the unknown front.

The eikonal equations (7), that are clearly imposed on the boundary $\left\{x_{2}=0\right\}$ by (4a)-(4b)(4c), ensure that the matrices $A_{2}\left(U^{ \pm}\right)-\partial_{t} \Phi^{ \pm}-\partial_{x_{1}} \Phi^{ \pm} A_{1}\left(U^{ \pm}\right)$have a constant rank in the whole domain $\left\{x_{2} \geq 0\right\}$, and not only on the boundary. This constant rank property was crucial in [12] to perform a Kreiss' type symmetrizers construction and to derive a priori estimates. We refer for instance to $[16,26,35,36]$ for various aspects of this constant rank condition in hyperbolic characteristic boundary value problems.

With an obvious definition, the equations (3) can be rewritten in the compact form:

$$
\mathbb{L}\left(U^{+}, \Phi^{+}\right)=\mathbb{L}\left(U^{-}, \Phi^{-}\right)=0 .
$$

For later use, it is also convenient to write the nonlinear operator $\mathbb{L}$ under the form $\mathbb{L}(U, \Phi)=$ $L(U, \Phi) U$. In other words, we have set:

$$
L(U, \Phi) V:=\partial_{t} V+A_{1}(U) \partial_{x_{1}} V+\frac{1}{\partial_{x_{2}} \Phi}\left(A_{2}(U)-\partial_{t} \Phi-\partial_{x_{1}} \Phi A_{1}(U)\right) \partial_{x_{2}} V .
$$

In the same way, the boundary conditions (4) can be rewritten in the compact form:

$$
\begin{aligned}
& \Phi_{\left.\right|_{x_{2}=0}}^{+}=\Phi_{\left.\right|_{x_{2}=0}}^{-}=\varphi, \\
& \mathbb{B}\left(U_{\left.\right|_{x_{2}}=0}^{+}, U_{\left.\right|_{x_{2}}=0}^{-}, \varphi\right)=0 .
\end{aligned}
$$

$4^{\text {e }}$ SÉRIE - TOME $41-2008-\mathrm{N}^{\mathrm{o}} 1$ 
The reader should keep in mind that the nonlinear equations (8) and (10) are supplemented with the initial conditions (5), and with the constraints (6) and (7).

There exist many simple solutions of (8), (10), (6), and (7), that correspond for the Euler equations (1) in the original variables to stationary rectilinear vortex sheets:

$$
(\rho, \mathbf{u})= \begin{cases}(\bar{\rho}, \bar{v}, 0), & \text { if } x_{2}>0, \\ (\bar{\rho},-\bar{v}, 0), & \text { if } x_{2}<0,\end{cases}
$$

where $\bar{\rho}, \bar{v} \in \mathbb{R}, \bar{\rho}>0$. Up to Galilean transformations, every rectilinear vortex sheet has this form. In the straightened variables, these stationary vortex sheets correspond to the following smooth stationary solution to (8), (10), (6), (7):

$$
\bar{U}^{ \pm} \equiv(\bar{\rho}, \pm \bar{v}, 0), \quad \bar{\Phi}^{ \pm}(t, x) \equiv \pm x_{2}, \quad \bar{\varphi} \equiv 0 .
$$

The corresponding constant $\kappa$ in (6) equals 1 . In this paper, we shall assume $\bar{v}>0$, but the opposite case can be dealt with in the same way.

Our goal is to construct solutions to the nonlinear system (8), (10), (6) and (7), with initial data (5) that are close to the stationary solution (11). (We expect that the solution remains close to the constant stationary solution.) This is a nonlinear stability problem, and we wish to solve the nonlinear equations by solving a sequence of linearized problems. As detailed earlier, in the noncharacteristic uniformly stable case, that was first treated by Majda [24, 23], a standard Picard iteration is sufficient to solve the nonlinear problem. In the case of compressible vortex sheets, the so-called uniform stability condition is never satisfied, therefore one cannot prove a maximal estimate in Sobolev spaces for the linearized equations. In [12], we have proved that the supersonic condition $\bar{v}>\sqrt{2} c(\bar{\rho})$ implies an a priori estimate for the linearized equations. (See section 3 for a precise statement.) The a priori estimate indicates a loss of one tangential derivative from the source terms to the solution. The loss is fixed, and we can thus expect to solve the nonlinear problem by a Nash-Moser iteration scheme, see [2]. Recall that the Nash-Moser procedure was already used to construct other types of waves for multidimensional systems of conservation laws, see [1, 14]. However, the Nash-Moser procedure we shall use here is not completely standard, since the tame estimate for the linearized equations will be obtained under certain nonlinear constraints on the state about which we linearize. We thus need to make sure that these constraints are satisfied at each iteration step. Let us now state our main result:

Theorem 1. - Let $T>0$, and let $\mu \in \mathbb{N}$, with $\mu \geq 6$. Assume that the stationary solution defined by (11) satisfies the "supersonic" condition:

$$
\bar{v}>\sqrt{2} c(\bar{\rho}) .
$$

Assume that the initial data $\left(U_{0}^{ \pm}, \varphi_{0}\right)$ have the form $U_{0}^{ \pm}=\bar{U}^{ \pm}+\dot{U}_{0}^{ \pm}$, with $\dot{U}_{0}^{ \pm} \in$ $H^{\mu+15 / 2}\left(\mathbb{R}_{+}^{2}\right), \varphi_{0} \in H^{\mu+8}(\mathbb{R})$, and that they are compatible up to order $\mu+7$ in the sense of Definition 1 ( see section 4). Assume also that $\left(\dot{U}_{0}^{ \pm}, \varphi_{0}\right)$ have a compact support. Then, there exists $\delta>0$ such that, if $\left\|\dot{U}_{0}^{ \pm}\right\|_{H^{\mu+15 / 2}\left(\mathbb{R}_{+}^{2}\right)}+\left\|\varphi_{0}\right\|_{H^{\mu+8}(\mathbb{R})} \leq \delta$, then there exists a solution $U^{ \pm}=\bar{U}^{ \pm}+\dot{U}^{ \pm}, \Phi^{ \pm}= \pm x_{2}+\dot{\Phi}^{ \pm}, \varphi$ of (3), (4), (5), (6), (7), on the time interval $[0, T]$. This solution satisfies $\left(\dot{U}^{ \pm}, \dot{\Phi}^{ \pm}\right) \in H^{\mu}(] 0, T\left[\times \mathbb{R}_{+}^{2}\right)$, and $\varphi \in H^{\mu+1}(] 0, T[\times \mathbb{R})$.

Let us make a few remarks about some connected problems: 
REMARK 2. - Theorem I shows that the condition (12), that is known to be necessary and sufficient for weak spectral stability, is also sufficient for nonlinear well-posedness. We prove that, if the initial vortex sheet at time zero is a sufficiently small perturbation of (11), (12), the solution of the nonlinear vortex sheet problem exists on a given time interval. The smallness condition ensures that singularities cannot form on this time interval. In particular, the formation of kink modes (as detailed in [3]) can occur only after the time T. Recall that the "supersonic" condition (12) also plays a crucial role in the analysis of [3].

The compatibility conditions ( see Definition 1 in section 4) are strong conditions imposed in order that only the desired contact discontinuity is generated by the initial discontinuity, similarly to [23, 1, 14]. The general Riemann problem, with all kinds of singularities (shocks, rarefaction waves, contact discontinuities ...) coming out from the initial discontinuity, is still an open problem. See however [18] for the case of analytic data.

For the sake of simplicity, we shall detail the proof of Theorem 1 when the support of $\varphi_{0}$ is included in $[-1,1]$, and the support of $\dot{U}_{0}^{ \pm}$is included in $\left\{x_{2} \geq 0, \sqrt{x_{1}^{2}+x_{2}^{2}} \leq 1\right\}$. Thanks to the finite speed of propagation of the Euler equations and the eikonal equation, the corresponding solution $\dot{U}^{ \pm}, \dot{\Phi}^{ \pm}, \varphi$ will have a compact support:

$$
\operatorname{Supp}\left(\dot{U}^{ \pm}, \dot{\Phi}^{ \pm}\right) \subset\left\{t \in[0, T], x_{2} \geq 0, \sqrt{x_{1}^{2}+x_{2}^{2}} \leq R\right\}, \operatorname{Supp} \varphi \subset\left\{t \in[0, T],\left|x_{1}\right| \leq R\right\},
$$

where $R$ is large enough.

We now detail the main steps of the proof of Theorem 1, and the way this article is organized. The main building block for the proof is to obtain the solvability of the linearized equations with a tame estimate. This is done in section 3. The basic a priori estimate in $L^{2}$ was derived in [12]. Using this first piece of information, the solvability of the linearized equations relies on two main steps. First, we need to check the solvability of the linearized equations in $L^{2}$, then we need to prove the tame estimate in Sobolev spaces of arbitrary regularity. We shall show the solvability in $L^{2}$ by applying the result of [10], so we only need to construct a dual problem that also satisfies an a priori estimate with a loss of one tangential derivative. The definition of the dual problem requires some care because the boundary is characteristic so the number of dual boundary conditions is important. To prove the tame estimate, we follow the classical path, see e.g. the works by Rauch and Massey [33] for noncharacteristic problems, and by Majda and Osher [26] for characteristic problems. However, our case is much less favorable than in all previous works because our estimates involve a loss of one derivative with respect to the source terms (both in the interior and on the boundary). Since the method in $[33,26]$ consists in treating all commutators as source terms, it is not clear at first sight that this method can be extended to such problems where losses of derivatives occur. Furthermore, in our problem, the boundary is characteristic so if we follow the procedure of [26], we would need to introduce conormal Sobolev spaces (where two tangential derivatives count as one normal derivative). Hopefully this can be avoided here by using the specific structure of the Euler equations, as already shown in [39, 34]. In section 3, we shall show that the usual method of [33] is flexible enough to handle the loss of derivatives, and we shall obtain a tame estimate in the usual Sobolev spaces. The adaptation of the classical commutation method to our weakly stable situation is the second main step of the proof.

$4^{\mathrm{e}}$ SÉRIE - TOME $41-2008-\mathrm{N}^{\mathrm{o}} 1$ 
In section 4, we detail the compatibility conditions for the initial data. Starting from compatible initial data, we construct an approximate solution, which reduces the original problem to solving a new nonlinear system with zero initial data. Our compatibility conditions are not entirely standard because we have thought it convenient to start with an approximate solution that satisfies the Rankine-Hugoniot conditions. We thus need to add a technical restriction that comes from a trace lemma for Sobolev spaces in a quarter space. Nevertheless, the analysis of section 4 follows closely the earlier work [33], see also [23, 29]. The introduction of the approximate solution reduces the problem to a new system with zero initial data. In section 5, we describe the iteration scheme that will be used to solve this reduced problem. Our Nash-Moser scheme follows the description in [2] but also incorporates the specific constraints that need to be satisfied at each iteration step. In section 6, we shall collect all the estimates that are needed in section 7 to prove the convergence of the scheme. Summing up, the main points consist in $i$ ) showing $L^{2}$ well-posedness, $i i$ ) adapting the usual commutation method for tame estimates to our weakly stable problem that involves a loss of derivatives, and iii) incorporating the nonlinear constraints in the Nash-Moser procedure.

Appendices A and B are devoted to the application of our technique to similar nonlinear free boundary hyperbolic problems, that is, the existence of weakly stable shocks in isentropic gas dynamics, and the existence of isothermal liquid-vapor phase transitions in a van der Waals fluid. We shall not detail the proof of our results, but rather explain how the main steps $i$ ), ii), iii) of our method can be handled in these situations that are technically easier to deal with (compared to the study of vortex sheets, the main advantage of these two other situations is the fact that the boundary is noncharacteristic).

It might be possible to prove Theorem 1 by using a standard Nash-Moser procedure, without having to ensure nonlinear constraints at each iteration step. However, any iteration will rely on a tame estimate for the linearized problem. For the linearized problem we consider here the boundary is characteristic, and it seems that the only technique available to prove an estimate is to construct Kreiss' type symmetrizers in the spirit of Majda and Osher's work [26]. This technique requires that the boundary matrix has a constant rank on a neighborhood of the boundary, and not only at the boundary. For our problem, this constant rank property is ensured by the eikonal equations (7). This nonlinear constraint is the major obstacle to using a standard Nash-Moser iteration. We shall thus need to introduce an intermediate step where the sequence of approximate solutions will be "projected" on the set of functions that satisfy the nonlinear constraints.

\section{Tame estimates for the linearized equations}

\subsection{Weighted spaces and norms}

To prove Theorem 1, we are going to use weighted Sobolev spaces that we introduce right now. First we define the half-space $\Omega:=\left\{\left(t, x_{1}, x_{2}\right) \in \mathbb{R}^{3} / x_{2}>0\right\}=\mathbb{R}^{2} \times \mathbb{R}^{+}$. The boundary $\omega:=\partial \Omega$ is identified to $\mathbb{R}^{2}$. We denote the usual norm of $L^{2}\left(\mathbb{R}^{2}\right)$, resp. $L^{2}(\Omega)$, by $\|\cdot\|_{L^{2}\left(\mathbb{R}^{2}\right)}$, resp. $\|\cdot\|_{L^{2}(\Omega)}$. For all real number $s$ and all $\gamma \geq 1$, we define the space:

$$
H_{\gamma}^{s}\left(\mathbb{R}^{2}\right):=\left\{u \in \mathcal{D}^{\prime}\left(\mathbb{R}^{2}\right) / \exp (-\gamma t) u \in H^{s}\left(\mathbb{R}^{2}\right)\right\}
$$


It is equipped with the obvious norm $\|u\|_{H_{\gamma}^{s}\left(\mathbb{R}^{2}\right)}:=\|\exp (-\gamma t) u\|_{H^{s}\left(\mathbb{R}^{2}\right)}$. The space $L^{2}\left(\mathbb{R}^{+} ; H_{\gamma}^{s}\left(\mathbb{R}^{2}\right)\right)$ is equipped with the obvious norm:

$$
\|u\|_{L^{2}\left(H_{\gamma}^{s}\right)}^{2}:=\int_{0}^{+\infty}\left\|u\left(\cdot, x_{2}\right)\right\|_{H_{\gamma}^{s}\left(\mathbb{R}^{2}\right)}^{2} d x_{2} .
$$

For all real number $T$ (in particular when $T>0$ ), we let $\Omega_{T}$ and $\omega_{T}$ denote the sets $\omega_{T}:=$ ]$-\infty, T\left[\times \mathbb{R}\right.$, and $\Omega_{T}:=\omega_{T} \times \mathbb{R}^{+}$. For all integer $m$, and all $\gamma \geq 1$, we define the weighted Sobolev space $H_{\gamma}^{m}\left(\Omega_{T}\right)$ as:

$$
H_{\gamma}^{m}\left(\Omega_{T}\right):=\left\{u \in \mathcal{D}^{\prime}\left(\Omega_{T}\right) / \exp (-\gamma t) u \in H^{m}\left(\Omega_{T}\right)\right\} .
$$

The definition of $H_{\gamma}^{m}\left(\omega_{T}\right)$ is similar. The norm on $H_{\gamma}^{m}\left(\Omega_{T}\right)$ may be defined by:

$$
\|u\|_{H_{\gamma}^{m}\left(\Omega_{T}\right)}:=\sum_{|\alpha| \leq m} \gamma^{m-|\alpha|}\left\|\mathrm{e}^{-\gamma t} \partial^{\alpha} u\right\|_{L^{2}\left(\Omega_{T}\right)},
$$

which is equivalent to the norm $\left\|\mathrm{e}^{-\gamma t} u\right\|_{H^{m}\left(\Omega_{T}\right)}$. The constant in the equivalence is independent of $\gamma \geq 1$, and $T$. The norm on $H_{\gamma}^{m}\left(\omega_{T}\right)$ is defined in the same way. Thus, the space $L^{2}\left(\mathbb{R}^{+} ; H_{\gamma}^{m}\left(\omega_{T}\right)\right)$ is equipped with the norm:

$$
\|u\|_{L^{2}\left(\mathbb{R}^{+} ; H_{\gamma}^{m}\left(\omega_{T}\right)\right)}:=\sum_{\alpha_{0}+\alpha_{1} \leq m} \gamma^{m-\alpha_{0}-\alpha_{1}}\left\|\mathrm{e}^{-\gamma t} \partial_{t}^{\alpha_{0}} \partial_{x_{1}}^{\alpha_{1}} u\right\|_{L^{2}\left(\Omega_{T}\right)} .
$$

This is an anisotropic Sobolev space where one measures only the tangential regularity (tangential means tangential with respect to the boundary $\left.\left\{x_{2}=0\right\}\right)$.

REMARK 3. - For functions $u(t, x)$ that are supported in a strip $\left\{t \in\left[T_{1}, T_{2}\right]\right\}, T_{1}<T_{2}$, one has $u \in H_{\gamma}^{m}\left(\Omega_{T_{2}}\right)$ if and only if $u \in H^{m}\left(\Omega_{T_{2}}\right)$, and the norms are equivalent. However, the constant in the equivalence of the norms heavily depends on $\gamma$. This is the reason why it will be more convenient to derive a tame estimate in the $H_{\gamma}^{m}$ norm, that is defined by (13).

Eventually, for $1 \leq p<+\infty$, and $T \in \mathbb{R}$, the space $L_{\gamma}^{p}\left(\Omega_{T}\right)$ is the set of measurable functions $u$ such that $\mathrm{e}^{-2 \gamma t / p} u$ belongs to $L^{p}\left(\Omega_{T}\right)$. The norm in $L_{\gamma}^{p}\left(\Omega_{T}\right)$ is the obvious one. There is a similar definition with $\omega_{T}$ instead of $\Omega_{T}$.

\subsection{The (effective) linearized equations}

We introduce the linearized equations around a state that is given as a perturbation of the stationary solution (11). More precisely, let us consider some functions:

$$
U_{r}=\bar{U}^{+}+\dot{U}_{r}(t, x), \quad U_{l}=\bar{U}^{-}+\dot{U}_{l}(t, x), \quad \Phi_{r}=x_{2}+\dot{\Phi}_{r}(t, x), \quad \Phi_{l}=-x_{2}+\dot{\Phi}_{l}(t, x) .
$$

The index $r$ (resp. $l$ ) denotes the state on the right (resp. on the left) of the interface before the change of variables. We assume that the perturbations $\dot{U}_{r, l}, \dot{\Phi}_{r, l}$ have a compact support:

$$
\operatorname{Supp}\left(\dot{U}_{r, l}, \dot{\Phi}_{r, l}\right) \subset\left\{t \in[-T, 2 T], x_{2} \geq 0, \sqrt{x_{1}^{2}+x_{2}^{2}} \leq R_{0}\right\} .
$$

$4^{\text {e }}$ SÉRIE - TOME $41-2008-\mathrm{N}^{\mathrm{o}} 1$ 
The smoothness of these perturbations will be chosen later on. We also assume that these quantities satisfy the boundary conditions (4), that is:

$$
\begin{aligned}
\Phi_{\left.r\right|_{x_{2}=0}}=\Phi_{l_{\left.\right|_{x_{2}}=0}} & =\varphi, \\
\left(v_{r}-v_{l}\right)_{\left.\right|_{x_{2}=0}} \partial_{x_{1}} \varphi-\left(u_{r}-u_{l}\right)_{\left.\right|_{x_{2}=0}} & =0, \\
\partial_{t} \varphi+v_{\left.r\right|_{x_{2}=0}} \partial_{x_{1}} \varphi-u_{\left.r\right|_{x_{2}=0}} & =0, \\
\left(\rho_{r}-\rho_{l}\right)_{\left.\right|_{x_{2}=0}} & =0 .
\end{aligned}
$$

Eventually, we assume that the functions $\Phi_{r, l}$ satisfy the eikonal equations:

$$
\partial_{t} \Phi_{r}+v_{r} \partial_{x_{1}} \Phi_{r}-u_{r}=\partial_{t} \Phi_{l}+v_{l} \partial_{x_{1}} \Phi_{l}-u_{l}=0
$$

together with:

$$
\partial_{x_{2}} \Phi_{r} \geq \kappa_{0}, \quad \partial_{x_{2}} \Phi_{l} \leq-\kappa_{0},
$$

for a suitable constant $\left.\kappa_{0} \in\right] 0,1\left[\right.$, in the whole closed half-space $\left\{x_{2} \geq 0\right\}$. Note that (18) can be deduced from a "smallness" condition on the perturbations $\dot{\Phi}_{r, l}$. This will be used in the following sections.

Let us consider some families $U_{s}^{ \pm}=U_{r, l}+s V_{ \pm}, \Phi_{s}^{ \pm}=\Phi_{r, l}+s \Psi_{ \pm}$, where $s$ is a small parameter. Recalling that the nonlinear operator $\mathbb{L}$ is defined in (8), we compute the linearized equations around the state $U_{r, l}, \Phi_{r, l}$ :

$$
\mathbb{L}^{\prime}\left(U_{r, l}, \Phi_{r, l}\right)\left(V_{ \pm}, \Psi_{ \pm}\right):=\left.\frac{d}{d s} \mathbb{L}\left(U_{s}^{ \pm}, \Phi_{s}^{ \pm}\right)\right|_{s=0}=f_{ \pm} .
$$

The structure of the linearized equations has been analyzed in [1]. Introducing the "good unknown":

$$
\dot{V}_{+}:=V_{+}-\frac{\Psi_{+}}{\partial_{x_{2}} \Phi_{r}} \partial_{x_{2}} U_{r}, \quad \dot{V}_{-}:=V_{-}-\frac{\Psi_{-}}{\partial_{x_{2}} \Phi_{l}} \partial_{x_{2}} U_{l},
$$

we have (see $[1,29]$ for some comments):

$$
\begin{gathered}
\mathbb{L}^{\prime}\left(U_{r}, \Phi_{r}\right)\left(V_{+}, \Psi_{+}\right)=L\left(U_{r}, \Phi_{r}\right) \dot{V}_{+}+C\left(U_{r}, \Phi_{r}\right) \dot{V}_{+}+\frac{\Psi_{+}}{\partial_{x_{2}} \Phi_{r}} \partial_{x_{2}}\left\{\mathbb{L}\left(U_{r}, \Phi_{r}\right)\right\}=f_{+}, \\
\mathbb{L}^{\prime}\left(U_{l}, \Phi_{l}\right)\left(V_{-}, \Psi_{-}\right)=L\left(U_{l}, \Phi_{l}\right) \dot{V}_{-}+C\left(U_{l}, \Phi_{l}\right) \dot{V}_{-}+\frac{\Psi_{-}}{\partial_{x_{2}} \Phi_{l}} \partial_{x_{2}}\left\{\mathbb{L}\left(U_{l}, \Phi_{l}\right)\right\}=f_{-},
\end{gathered}
$$

where, for all smooth enough functions $(U, \Phi)$, the matrix $C(U, \Phi)$ is defined as follows:

$$
C(U, \Phi) X:=\left[d A_{1}(U) X\right] \partial_{x_{1}} U+\frac{1}{\partial_{x_{2}} \Phi}\left\{\left[d A_{2}(U) X\right]-\partial_{x_{1}} \Phi\left[d A_{1}(U) X\right]\right\} \partial_{x_{2}} U
$$

In particular, the matrices $C\left(U_{r, l}, \Phi_{r, l}\right)$ are $\mathcal{C}^{\infty}$ functions of $\left(\dot{U}_{r, l}, \nabla \dot{U}_{r, l}, \nabla \dot{\Phi}_{r, l}\right)$ that vanish when $\left(\dot{U}_{r, l}, \nabla \dot{U}_{r, l}, \nabla \dot{\Phi}_{r, l}\right)=0$.

In view of the results proved in [1, 14], we neglect the zero order term in $\Psi_{+}, \Psi_{-}$in the linearized equations (20), and we thus consider the effective linear operators:

$$
\begin{array}{r}
\mathbb{L}_{e}^{\prime}\left(U_{r}, \Phi_{r}\right) \dot{V}_{+}:=L\left(U_{r}, \Phi_{r}\right) \dot{V}_{+}+C\left(U_{r}, \Phi_{r}\right) \dot{V}_{+}=f_{+}, \\
\mathbb{L}_{e}^{\prime}\left(U_{l}, \Phi_{l}\right) \dot{V}_{-}:=L\left(U_{l}, \Phi_{l}\right) \dot{V}_{-}+C\left(U_{l}, \Phi_{l}\right) \dot{V}_{-}=f_{-} .
\end{array}
$$

In the next sections, we shall show how to solve the nonlinear problem (8), (10), (6), (7) by means of a sequence of linear equations of the form (22). The remaining terms in (20) will 
be considered as error terms at each iteration step. The linearized front $\Psi_{ \pm}$does not appear anymore in these equations. This is crucial to derive energy estimates and to define a dual problem.

It is clear that the effective linearized equations (22) form a symmetrizable hyperbolic system. For instance, a Friedrichs symmetrizer for the operator $\mathbb{L}_{e}^{\prime}\left(U_{r, l}, \Phi_{r, l}\right)$ is given by:

$$
S_{r, l}:=\operatorname{diag}\left(\frac{p^{\prime}\left(\rho_{r, l}\right)}{\rho_{r, l}}, \rho_{r, l}, \rho_{r, l}\right) .
$$

Using the eikonal equations (17), we compute:

$\frac{S_{r}}{\partial_{x_{2}} \Phi_{r}}\left(A_{2}\left(U_{r}\right)-\partial_{t} \Phi_{r}-\partial_{x_{1}} \Phi_{r} A_{1}\left(U_{r}\right)\right)=\frac{1}{\partial_{x_{2}} \Phi_{r}}\left(\begin{array}{ccc}0 & -p^{\prime}\left(\rho_{r}\right) \partial_{x_{1}} \Phi_{r} & p^{\prime}\left(\rho_{r}\right) \\ -p^{\prime}\left(\rho_{r}\right) \partial_{x_{1}} \Phi_{r} & 0 & 0 \\ p^{\prime}\left(\rho_{r}\right) & 0 & 0\end{array}\right)$,

and we thus expect to control the traces of the components $\dot{V}_{+, 1}$, and $\left(\dot{V}_{+, 3}-\partial_{x_{1}} \Phi_{r} \dot{V}_{+, 2}\right)$ on the boundary $\left\{x_{2}=0\right\}$. In the same way, we expect to control the traces of the components $\dot{V}_{-, 1}$, and $\left(\dot{V}_{-, 3}-\partial_{x_{1}} \Phi_{l} \dot{V}_{-, 2}\right)$ on the boundary. We therefore introduce the following "trace operator":

$$
\mathbb{P}(\varphi) \dot{V}_{ \pm\left.\right|_{x_{2}=0}}:=\left.\left(\begin{array}{c}
\dot{V}_{ \pm, 1} \\
\dot{V}_{ \pm, 3}-\partial_{x_{1}} \varphi \dot{V}_{ \pm, 2}
\end{array}\right)\right|_{x_{2}=0} .
$$

This operator will be used in the energy estimates for the linearized equations. Its image can be understood as the "noncharacteristic" part of the vector $\dot{V}$.

We now turn to the linearized boundary conditions. Let us introduce the matrices:

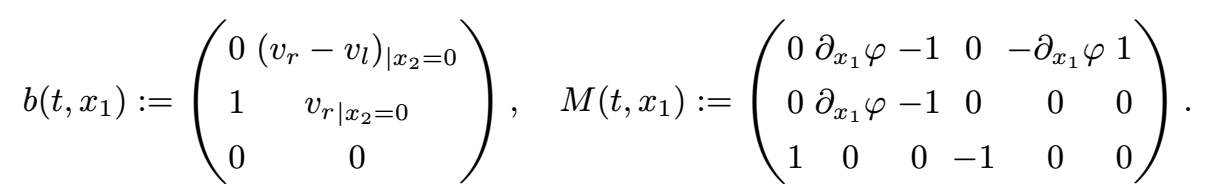

Let us denote $V=\left(V_{+}, V_{-}\right)^{T}$, and $\nabla \psi=\left(\partial_{t} \psi, \partial_{x_{1}} \psi\right)^{T}$. Then the linearization of the boundary conditions (4) reads:

$$
\begin{array}{r}
\left.\Psi_{+}\right|_{x_{2}=0}=\left.\Psi_{-}\right|_{x_{2}=0}=\psi, \\
\mathbb{B}^{\prime}\left(U_{r, l}, \Phi_{r, l}\right)\left(V_{\left.\right|_{x_{2}=0}}, \psi\right)=b \nabla \psi+M V_{\left.\right|_{x_{2}=0}}=g .
\end{array}
$$

In terms of the good unknown $\dot{V}$ defined by (19), the linearized boundary conditions read:

$$
\begin{array}{r}
\left.\Psi_{+}\right|_{x_{2}=0}=\left.\Psi_{-}\right|_{x_{2}=0}=\psi, \\
\mathbb{B}_{e}^{\prime}\left(U_{r, l}, \Phi_{r, l}\right)\left(\dot{V}_{\left.\right|_{x_{2}}=0}, \psi\right):=b \nabla \psi+\underbrace{\left.M\left(\begin{array}{c}
\partial_{x_{2}} U_{r} / \partial_{x_{2}} \Phi_{r} \\
\partial_{x_{2}} U_{l} / \partial_{x_{2}} \Phi_{l}
\end{array}\right)\right|_{x_{2}=0}}_{b_{\sharp}} \psi+M \dot{V}_{\left.\right|_{x_{2}}=0}=g .
\end{array}
$$

We observe that the operator $\mathbb{B}_{e}^{\prime}$ only involves $\left.\mathbb{P}(\varphi) \dot{V}_{ \pm}\right|_{x_{2}=0}$, where $\mathbb{P}(\varphi)$ is defined by (23), see the expression of the matrix $M$ in (24). 


\subsection{The basic $L^{2}$ a priori estimate}

We recall the $L^{2}$ a priori estimate for (22), (25) that we have derived in [12]. We assume that the perturbations satisfy:

(26)

$\dot{U}_{r}, \dot{U}_{l} \in W^{2, \infty}(\Omega), \quad \dot{\Phi}_{r}, \dot{\Phi}_{l} \in W^{3, \infty}(\Omega), \quad\left\|\left(\dot{U}_{r}, \dot{U}_{l}\right)\right\|_{W^{2, \infty}(\Omega)}+\left\|\left(\dot{\Phi}_{r}, \dot{\Phi}_{l}\right)\right\|_{W^{3, \infty}(\Omega)} \leq K$,

where $K$ is a positive constant. Then the following result holds:

THeOREm 2 ([12]). - Assume that the particular solution defined by (11) satisfies the "supersonic" condition (12), and that the perturbations $\dot{U}_{r, l}, \dot{\Phi}_{r, l}$ satisfy (15), (16), (17), (18) and (26). There exist some positive constants $K_{0}>0, C_{0}>0$, and $\gamma_{0} \geq 1$, such that if $K \leq K_{0}$, and $\gamma \geq \gamma_{0}$, then for all $(\dot{V}, \psi) \in H_{\gamma}^{2}(\Omega) \times H_{\gamma}^{2}\left(\mathbb{R}^{2}\right)$ the following a priori estimate holds:

$$
\begin{aligned}
& \gamma\|\dot{V}\|_{L_{\gamma}^{2}(\Omega)}^{2}+\left\|\mathbb{P}(\varphi) \dot{V}_{\left.\right|_{x_{2}=0}}\right\|_{L_{\gamma}^{2}\left(\mathbb{R}^{2}\right)}^{2}+\|\psi\|_{H_{\gamma}^{1}\left(\mathbb{R}^{2}\right)}^{2} \\
\leq & C_{0}\left(\frac{1}{\gamma^{3}}\left\|\left(\mathbb{L}_{e}^{\prime}\left(U_{r}, \Phi_{r}\right) \dot{V}_{+}, \mathbb{L}_{e}^{\prime}\left(U_{l}, \Phi_{l}\right) \dot{V}_{-}\right)\right\|_{L^{2}\left(H_{\gamma}^{1}\right)}^{2}+\frac{1}{\gamma^{2}}\left\|\mathbb{B}_{e}^{\prime}\left(U_{r, l}, \Phi_{r, l}\right)\left(\left.\dot{V}\right|_{x_{2}=0}, \psi\right)\right\|_{H_{\gamma}^{1}\left(\mathbb{R}^{2}\right)}^{2}\right) .
\end{aligned}
$$

The operators $\mathbb{P}(\varphi), \mathbb{L}_{e}^{\prime}$, and $\mathbb{B}_{e}^{\prime}$ are defined in (23), (22) and (25).

Before going on, we make a few comments:

REMARK 4. - The result of Theorem 2 is independent of the lower order term in the linearized equations. More precisely, if we consider two matrices $D_{r, l} \in W^{1, \infty}(\Omega)$, then the same result as in Theorem 2 holds with the matrices $C\left(U_{r, l}, \Phi_{r, l}\right)$, see (21)-(22), replaced by $D_{r, l}$. The corresponding constants will only depend on the $W^{1, \infty}$ norm of $D_{r, l}$.

There is no loss of generality in assuming $K_{0} \leq 1 / 2$ in Theorem 2. In particular, (18) is satisfied with $\kappa_{0}=1 / 2$. From now on we shall therefore forget about the constraint (18).

One requirement on $K_{0}$ is that at each point $\left(t, x_{1}, 0\right)$ of the boundary $\left\{x_{2}=0\right\}$, the frozen state $\left(U_{r, l}, \Phi_{r, l}\right)\left(t, x_{1}, 0\right)$ defines a weakly stable rectilinear vortex sheet (that is, it satisfies (12) up to an appropriate change of Galilean frame). This ensures that at each point of the boundary, the weak Lopatinskii condition is satisfied. Such a restriction is possible, because the domain of weak stability is defined by an "open" condition on the states that satisfy the Rankine-Hugoniot conditions.

We now turn to the first main step of our analysis, namely proving that the linearized equations are well-posed.

\subsection{Well-posedness of the linearized equations}

In this section, we show how to apply the well-posedness result of [10] to the linearized equations (22), (25). In view of the result of [10], we only need to check that there exists a dual problem that satisfies an a priori estimate with a loss of one tangential derivative. This is because the coefficients of the linearized operators satisfy the symmetrizability and regularity assumptions of [10]. The first task consists in defining a dual problem for (22), (25). 
On the boundary $\left\{x_{2}=0\right\}$, the matrix in front of the normal derivative $\partial_{x_{2}}$ in the operator $\mathbb{L}_{e}^{\prime}\left(U_{r, l}, \Phi_{r, l}\right)$ equals:

$$
\begin{aligned}
\left.\frac{1}{\partial_{x_{2}} \Phi_{r, l}}\left\{A_{2}\left(U_{r, l}\right)-\partial_{t} \Phi_{r, l}-\partial_{x_{1}} \Phi_{r, l} A_{1}\left(U_{r, l}\right)\right\}\right|_{x_{2}=0}= & \\
& \frac{1}{\left.\partial_{x_{2}} \Phi_{r, l}\right|_{x_{2}=0}}\left(\begin{array}{ccc}
0 & -\rho \partial_{x_{1}} \varphi & \rho \\
-\frac{c^{2}}{\rho} \partial_{x_{1}} \varphi & 0 & 0 \\
\frac{c^{2}}{\rho} & 0 & 0
\end{array}\right),
\end{aligned}
$$

where $\rho$ denotes the common trace of $\rho_{r}$ and $\rho_{l}$, see (16), $c$ is a short notation for the sound speed $c(\rho)$, and $\varphi$ is the common trace of $\Phi_{r}$ and $\Phi_{l}$. Recall that the matrix $M$ is defined by (24). In particular, one has $M \in W^{2, \infty}\left(\mathbb{R}^{2}\right)$. We define the following matrices:

$$
\begin{aligned}
& N:=\left(\begin{array}{cccccc}
0 & \partial_{x_{1}} \varphi & -1 & 0 & \partial_{x_{1}} \varphi & -1 \\
0 & \partial_{x_{1}} \varphi & -1 & 0 & 0 & 0 \\
1 & 0 & 0 & 1 & 0 & 0
\end{array}\right), \\
& M_{1}:=\left(\begin{array}{cccccc}
0 & 0 & 0 & \frac{\rho}{\partial_{x_{2}} \Phi_{l}} & 0 & 0 \\
\frac{-\rho}{\partial_{x_{2}} \Phi_{r}} & 0 & 0 & \frac{-\rho}{\partial_{x_{2}} \Phi_{l}} & 0 & 0 \\
0 & \frac{-c^{2} \partial_{x_{1}} \varphi}{2 \rho \partial_{x_{2}} \Phi_{r}} & \frac{c^{2}}{2 \rho \partial_{x_{2}} \Phi_{r}} & 0 & \frac{c^{2} \partial_{x_{1}} \varphi}{2 \rho \partial_{x_{2}} \Phi_{l}} & \frac{-c^{2}}{2 \rho \partial_{x_{2}} \Phi_{l}}
\end{array}\right) \text {, } \\
& N_{1}:=\left(\begin{array}{cccccc}
0 & 0 & 0 & 0 & 0 & 0 \\
0 & 0 & 0 & 0 & 0 & 0 \\
0 \frac{-c^{2} \partial_{x_{1}} \varphi}{2 \rho \partial_{x_{2}} \Phi_{r}} & \frac{c^{2}}{2 \rho \partial_{x_{2}} \Phi_{r}} & 0 & \frac{c^{2} \partial_{x_{1}} \varphi}{2 \rho \partial_{x_{2}} \Phi_{l}} & \frac{-c^{2}}{2 \rho \partial_{x_{2}} \Phi_{l}}
\end{array}\right),
\end{aligned}
$$

where the derivatives of the functions $\partial_{x_{2}} \Phi_{r}, \partial_{x_{2}} \Phi_{l}$ are all evaluated on the boundary $\left\{x_{2}=0\right\}$. The reader will check that these matrices satisfy the relation:

$$
\left.\frac{1}{\partial_{x_{2}} \Phi_{r, l}}\left(A_{2}\left(U_{r, l}\right)-\partial_{t} \Phi_{r, l}-\partial_{x_{1}} \Phi_{r, l} A_{1}\left(U_{r, l}\right)\right)\right|_{x_{2}=0}=M_{1}^{T} M+N_{1}^{T} N
$$

Moreover, using (26), one has $M_{1}, N_{1}, N \in W^{2, \infty}\left(\mathbb{R}^{2}\right)$.

Following [29, page 61], we can define a dual problem for (22), (25), in the following way:

$$
\begin{cases}\mathbb{L}_{e}^{\prime}\left(U_{r}, \Phi_{r}\right)^{*} U_{+}=\tilde{f}_{+}, & x_{2}>0, \\ \mathbb{L}_{e}^{\prime}\left(U_{l}, \Phi_{l}\right)^{*} U_{-}=\widetilde{f}_{-}, & x_{2}>0, \\ N_{1} U_{\left.\right|_{x_{2}}=0}=0, & \\ \operatorname{div}\left(b^{T} M_{1} U_{\left.\right|_{x_{2}=0}}\right)-b_{\sharp}^{T} M_{1} U_{\left.\right|_{x_{2}=0}}=0, & \end{cases}
$$

where $N_{1}, M_{1}$ are defined in (28), $b$ is defined in (24), $b_{\sharp}$ is defined in (25), div denotes the divergence operator in $\mathbb{R}^{2}$ with respect to the variables $\left(t, x_{1}\right)$, and the dual operators $\mathbb{L}_{e}^{\prime}\left(U_{r, l}, \Phi_{r, l}\right)^{*}$ are the formal adjoints of $\mathbb{L}_{e}^{\prime}\left(U_{r, l}, \Phi_{r, l}\right)$. Recall that the dual problem is derived by integration by parts, and by using the relation (29) for the boundary terms. We refer to [7, 29] for the details. 
Let us note that the dual problem (30) has exactly two independent scalar boundary conditions, since the two first rows of the matrix $N_{1}$ are zero, see (28). This is compatible with the number of incoming characteristics, because each of the boundary matrices of the operators $\mathbb{L}_{e}^{\prime}\left(U_{r, l}, \Phi_{r, l}\right)^{*}$ have one positive, one negative, and one null eigenvalue. The hyperbolic system (30) thus has two incoming characteristics, and two outgoing characteristics.

In view of the result of [10], we only need to check that the dual problem (30) satisfies an a priori estimate with a loss of one tangential derivative. First of all, we can derive the so-called Lopatinskii determinant associated with the boundary conditions in (30). This amounts to freezing the coefficients at one point of the boundary, and to performing a normal modes analysis. We shall not give the details of this analysis, since the calculations are really similar to those that can be found in [37, page 222], or in [12, page 956]. The result is the following:

Lemma 1. - The dual problem (30) satisfies the backward weak Lopatinskii condition. Moreover, the associated Lopatinskii determinant is smooth (that is, $\mathcal{C}^{\infty}$ ) near its zeroes, and its zeroes are simple. (The zeroes coincide with the zeroes of the Lopatinskii determinant associated with the original problem.)

As a matter of fact, the Lopatinskii determinant is exactly equal to the Lopatinskii determinant of the original problem (22), (25) provided that the stable subspaces that are involved in the calculation are parametrized in a suitable way. Consequently, one can reproduce exactly the same analysis as in [12], and show that the dual problem satisfies an a priori estimate with a loss of one tangential derivative both on the boundary, and in the interior domain. The linearized equations (22), (25) thus satisfy all the symmetrizability, regularity and weak stability assumptions of [10]. We therefore have the following well-posedness result:

Theorem 3. - Let T $>0$. Assume that the stationary solution (11) satisfies (12), and that the perturbations $\dot{U}_{r, l}, \dot{\Phi}_{r, l}$ satisfy (15), (16), (17) and (26). There exist three positive constants $K_{0}>0, \gamma_{0} \geq 1$, and $C_{0}>0$, that do not depend on $T$, such that if $K \leq K_{0}$, then for all source terms $f_{+}, f_{-}$, and $g$ that satisfy:

$$
f_{ \pm} \in L^{2}\left(\mathbb{R}^{+} ; H^{1}\left(\omega_{T}\right)\right), \quad g \in H^{1}\left(\omega_{T}\right),
$$

and that vanish for $t<0$, there exists a unique $\left(\dot{V}_{+}, \dot{V}_{-}, \psi\right) \in L^{2}\left(\Omega_{T}\right) \times L^{2}\left(\Omega_{T}\right) \times H^{1}\left(\omega_{T}\right)$ such that $\mathbb{P}(\varphi) \dot{V}_{\left.\right|_{x_{2}=0}} \in L^{2}\left(\omega_{T}\right),\left(\dot{V}_{+}, \dot{V}_{-}, \psi\right)$ is a solution to:

$$
\begin{cases}\mathbb{L}_{e}^{\prime}\left(U_{r}, \Phi_{r}\right) \dot{V}_{+}=f_{+}, & t<T, x_{2}>0, \\ \mathbb{L}_{e}^{\prime}\left(U_{l}, \Phi_{l}\right) \dot{V}_{-}=f_{-}, & t<T, x_{2}>0, \\ \mathbb{B}_{e}^{\prime}\left(U_{r, l}, \Phi_{r, l}\right)\left(\dot{V}_{\left.\right|_{x_{2}}=0}, \psi\right)=g, & t<T,\end{cases}
$$

and $\left(\dot{V}_{+}, \dot{V}_{-}, \psi\right)$ vanishes for $t<0$. In addition, $\left(\dot{V}_{+}, \dot{V}_{-}\right) \in \mathcal{C}\left([0, T] ; L^{2}\left(\mathbb{R}_{+}^{2}\right)\right)$, and we have the following inequality for all $\gamma \geq \gamma_{0}$, and for all $t \in[0, T]$ :

$$
\begin{aligned}
\mathrm{e}^{-\gamma t}\|\dot{V}(t)\|_{L^{2}\left(\mathbb{R}_{+}^{2}\right)}+\sqrt{\gamma}\|\dot{V}\|_{L_{\gamma}^{2}\left(\Omega_{t}\right)} & +\left\|\mathbb{P}(\varphi) \dot{V}_{\left.\right|_{x_{2}=0}}\right\|_{L_{\gamma}^{2}\left(\omega_{t}\right)}+\|\psi\|_{H_{\gamma}^{1}\left(\omega_{t}\right)} \\
& \leq C_{0}\left(\frac{1}{\gamma^{3 / 2}}\left\|\left(f_{+}, f_{-}\right)\right\|_{L^{2}\left(H_{\gamma}^{1}\left(\omega_{t}\right)\right)}+\frac{1}{\gamma}\|g\|_{H_{\gamma}^{1}\left(\omega_{t}\right)}\right) .
\end{aligned}
$$


Theorem 3 states the well-posedness of (22), (25) in $L^{2}$ when the source terms belong to $L^{2}\left(H^{1}\right) \times H^{1}$. When dealing with quasilinear problems, this result is clearly not sufficient and we need to obtain a similar well-posedness result in a Sobolev space $H^{m}$, with $m$ large enough. As a matter of fact, when dealing with a Nash-Moser iteration, one needs a wellposedness result in the Sobolev space $H^{m}$, where $m$ is arbitrarily large. The crucial point is to obtain also a tame estimate in $H^{m}$ (roughly speaking, there is a fixed loss of derivatives with respect to the source terms and to the coefficients, and high norms are multiplied by low norms). The remaining of this section is devoted to obtaining this tame estimate.

The method is classical and dates back at least to [33] (see [26] for characteristic problems): we consider the linear equations (22), (25). We first change the equations in order to deal with a boundary matrix of the form:

$$
\mathbf{I}_{2}:=\left(\begin{array}{lll}
0 & 0 & 0 \\
0 & 1 & 0 \\
0 & 0 & 1
\end{array}\right) .
$$

This is possible because the boundary matrix has a constant rank in the whole domain $\Omega$. Then we estimate the tangential derivatives, and we can deduce an estimate for some of the normal derivatives. Observe that the boundary is characteristic so that the equations (22) enable us to control only 4 normal derivatives in terms of tangential derivatives. Actually, the missing normal derivatives are those of the tangential velocity (both on the right and on the left of the interface). Due to the special structure of the Euler equations, these normal derivatives can be estimated by computing the equation for the vorticity as already proved in [39, 34].

Of course, the derivation of energy estimates in Sobolev spaces is possible only when the coefficients have more regularity than what is stated in (26). This is of little consequence since, in a Nash-Moser iteration, the coefficients are very smooth at each iteration step.

To estimate the tangential derivatives of the solution to (22), (25), one commutes the equation with a tangential derivative and applies the Gagliardo-Nirenberg inequalities to estimate the commutator. These estimates are recalled in Appendix $\mathrm{C}$ at the end of this paper, together with other useful nonlinear estimates. It should be highlighted that our basic $L^{2}$ estimate involves a loss of one tangential derivative so we cannot simply treat all commutators as source terms. Moreover, all source terms need to be estimated in $L^{2}\left(H^{1}\right)$, instead of $L^{2}$ when one has a maximal estimate. The procedure thus requires more attention, because all source terms need to be absorbed, though being estimated in a higher norm. We shall show however that the procedure can be carried out to obtain the expected tame estimate. This is somehow surpriseless, but a detailed analysis cannot be avoided.

\subsection{An equivalent formulation of the linearized equations}

For convenience, we first transform the interior equations (22) in order to deal with a hyperbolic operator that has a constant diagonal boundary matrix. This transformation is detailed in [12, section 5.2], and we refer to this article for the details. The result is the following.

$4^{\text {e }}$ SÉRIE - TOME $41-2008-\mathrm{N}^{\mathrm{o}} 1$ 
There exists an invertible matrix $T(U, \nabla \Phi)$, that is a smooth function of its coefficients, such that if one defines the vectors:

$$
W_{+}:=T\left(U_{r}, \nabla \Phi_{r}\right) \dot{V}_{+}, \quad W_{-}:=T\left(U_{l}, \nabla \Phi_{l}\right) \dot{V}_{-},
$$

then these vectors solve the system:

$$
\begin{aligned}
& \mathbf{A}_{0}^{r} \partial_{t} W_{+}+\mathbf{A}_{1}^{r} \partial_{x_{1}} W_{+}+\mathbf{I}_{2} \partial_{x_{2}} W_{+}+\mathbf{C}^{r} W_{+}=F_{+}, \\
& \mathbf{A}_{0}^{l} \partial_{t} W_{-}+\mathbf{A}_{1}^{l} \partial_{x_{1}} W_{-}+\mathbf{I}_{2} \partial_{x_{2}} W_{-}+\mathbf{C}^{l} W_{-}=F_{-},
\end{aligned}
$$

where the matrices $\mathbf{A}_{0}^{r, l}, \mathbf{A}_{1}^{r, l}$ belong to $W^{2, \infty}(\Omega)$, and the matrix $\mathbf{C}^{r, l}$ belongs to $W^{1, \infty}(\Omega)$. Moreover $\mathbf{A}_{0}^{r, l}$ and $\mathbf{A}_{1}^{r, l}$ are $\mathcal{C}^{\infty}$ functions of their arguments $\left(\dot{U}_{r, l}, \nabla \dot{\Phi}_{r, l}\right)$, while $\mathbf{C}^{r, l}$ is a $\mathcal{C}^{\infty}$ function of its arguments $\left(\dot{U}_{r, l}, \nabla \dot{U}_{r, l}, \nabla \dot{\Phi}_{r, l}, \nabla^{2} \dot{\Phi}_{r, l}\right)$ that vanishes at the origin. The matrix $\mathbf{I}_{2}$ is defined in (31), and the source terms $F^{ \pm}$are defined by:

$$
F_{ \pm}(t, x)=\mathbf{A}_{0}^{r, l} T\left(U_{r, l}, \nabla \Phi_{r, l}\right) f_{ \pm}(t, x) .
$$

The system (33) is equivalent to (22) because $\mathbf{A}_{0}^{r, l}$ are invertible. Observe that the source terms in (33) differ from the source terms in (22) by an invertible matrix. Until the end of this section, we always keep the notation $F_{ \pm}$for the source terms in (33), while $f_{ \pm}$denote the source terms in (22).

Using the vector $W=\left(W_{+}, W_{-}\right)^{T}$, the linearized boundary conditions (25) become:

$$
\begin{array}{r}
\left.\Psi_{+}\right|_{x_{2}=0}=\left.\Psi_{-}\right|_{x_{2}=0}=\psi, \\
b \nabla \psi+b_{\sharp} \psi+\mathbf{M} W_{\left.\right|_{x_{2}=0}}=g,
\end{array}
$$

where we have set

$$
\mathbf{M}:=M\left(\begin{array}{cc}
T_{r}^{-1} & 0 \\
0 & T_{l}^{-1}
\end{array}\right) .
$$

The matrices $b$ and $\mathbf{M}$ belong to $W^{2, \infty}\left(\mathbb{R}^{2}\right)$, and the vector $b_{\sharp}$ belongs to $W^{1, \infty}\left(\mathbb{R}^{2}\right)$. Moreover, $b$ is a $\mathcal{C}^{\infty}$ function of $\left.\dot{U}_{r, l}\right|_{x_{2}=0}, \mathbf{M}$ is a $\mathcal{C}^{\infty}$ function of $\left(\left.\dot{U}_{r, l}\right|_{x_{2}=0}, \partial_{x_{1}} \varphi\right)$, and $b_{\sharp}$ is a $\mathcal{C}^{\infty}$ function of $\left(\left.\partial_{x_{2}} \dot{U}_{r, l}\right|_{x_{2}=0}, \partial_{x_{1}} \varphi,\left.\partial_{x_{2}} \dot{\Phi}_{r, l}\right|_{x_{2}=0}\right)$ that vanishes at the origin.

As detailed in [12], the new boundary conditions (35) only involve the "noncharacteristic" part of the vector $W=\left(W_{+}, W_{-}\right)^{T}$, that is, the sub-vector $W^{n c}:=\left(W_{+, 2}, W_{+, 3}, W_{-, 2}\right.$, $\left.W_{-, 3}\right)^{T}$. These are the components whose trace can be controlled on the boundary $\left\{x_{2}=0\right\}$. Consequently, we shall feel free to rewrite the product $\mathbf{M} W_{\left.\right|_{x_{2}=0}}$ as $\mathbf{M} W_{\left.\right|_{x_{2}=0}}^{n c}$, even though the dimensions of the matrices do not agree.

\subsection{A priori estimate of tangential derivatives}

In the new formulation (33), (35), the estimate of Theorem 3 gives the following:

$$
\begin{aligned}
\sqrt{\gamma}\|W\|_{L_{\gamma}^{2}\left(\Omega_{T}\right)}+\left\|W_{\left.\right|_{x_{2}}=0}^{n c}\right\|_{L_{\gamma}^{2}\left(\omega_{T}\right)}+ & \|\psi\|_{H_{\gamma}^{1}\left(\omega_{T}\right)} \\
& \leq C_{0}\left(\frac{1}{\gamma^{3 / 2}}\|F\|_{L^{2}\left(H_{\gamma}^{1}\left(\omega_{T}\right)\right)}+\frac{1}{\gamma}\|g\|_{H_{\gamma}^{1}\left(\omega_{T}\right)}\right),
\end{aligned}
$$

whenever $\gamma \geq \gamma_{0}, K \leq K_{0}$ and $K_{0}$ given by Theorem 3 .

From now on, we assume that the perturbations $\dot{U}_{r, l}$, and $\dot{\Phi}_{r, l}$ not only satisfy (26), but also belong to the space $H_{\gamma}^{k}(\Omega)$ for all integer $k$, and all $\gamma \geq 1$. Moreover, to avoid overloaded 
expressions, we shall feel free to write $\dot{U}, \dot{\Phi}$, instead of $\dot{U}_{r, l}, \dot{\Phi}_{r, l}$. The aim of this paragraph is to prove the following result:

Proposition 1. - Let $m \in \mathbb{N}, m \geq 1$, and let $T>0$. There exist two constants $C_{m}>0$ and $\gamma_{m} \geq 1$, that do not depend on $T$, such that for all $\gamma \geq \gamma_{m}$ and for all $(W, \psi) \in H_{\gamma}^{m+2}\left(\Omega_{T}\right) \times H_{\gamma}^{m+2}\left(\omega_{T}\right)$ solution to (33), (35), the following estimate holds:

$$
\begin{aligned}
\sqrt{\gamma} \| & W\left\|_{L^{2}\left(H_{\gamma}^{m}\left(\omega_{T}\right)\right)}+\right\| W_{\mid x_{2}=0}^{n c}\left\|_{H_{\gamma}^{m}\left(\omega_{T}\right)}+\right\| \psi \|_{H_{\gamma}^{m+1}\left(\omega_{T}\right)} \\
\leq & C_{m}\left\{\frac{1}{\gamma^{3 / 2}}\|F\|_{L^{2}\left(H_{\gamma}^{m+1}\left(\omega_{T}\right)\right)}+\frac{1}{\gamma}\|g\|_{H_{\gamma}^{m+1}\left(\omega_{T}\right)}\right. \\
& +\frac{1}{\gamma^{3 / 2}}\|W\|_{W^{1, \infty}\left(\Omega_{T}\right)}\|(\dot{U}, \nabla \dot{\Phi})\|_{H_{\gamma}^{m+2}\left(\Omega_{T}\right)} \\
& \left.+\frac{1}{\gamma}\left(\left\|W_{\mid x_{2}=0}^{n c}\right\|_{L^{\infty}\left(\omega_{T}\right)}+\|\psi\|_{W^{1, \infty}\left(\omega_{T}\right)}\right)\left\|\left(\dot{U}, \partial_{x_{2}} \dot{U}, \nabla \dot{\Phi}\right)_{\mid x_{2}=0}\right\|_{H_{\gamma}^{m+1}\left(\omega_{T}\right)}\right\} .
\end{aligned}
$$

The main point in Proposition 1 is to clarify the dependance of the estimate with respect to the coefficients, and to check that the loss of derivatives does not prevent from absorbing the commutators that appear in the calculations. This does not follow directly from the $L^{2}$ estimate of Theorem 3 because of the loss of derivatives.

Proof. - We consider an integer $\ell$ such that $1 \leq \ell \leq m$, and a tangential derivative $\partial^{\alpha}=$ $\partial_{t}^{\alpha_{0}} \partial_{x_{1}}^{\alpha_{1}}$, with $\ell=|\alpha|$. Starting from (33), we compute:

$$
\begin{aligned}
\mathbf{A}_{0}^{r} \partial_{t} \partial^{\alpha} W_{+}+\mathbf{A}_{1}^{r} \partial_{x_{1}} \partial^{\alpha} W_{+}+\mathbf{I}_{2} \partial_{x_{2}} \partial^{\alpha} W_{+}+\mathbf{C}^{r} \partial^{\alpha} W_{+} \\
\quad+\sum_{|\beta|=1, \beta \leq \alpha} \star\left[\partial^{\beta} \mathbf{A}_{0}^{r} \partial^{\alpha-\beta} \partial_{t} W_{+}+\partial^{\beta} \mathbf{A}_{1}^{r} \partial^{\alpha-\beta} \partial_{x_{1}} W_{+}\right] \\
=\partial^{\alpha} F_{+}+\sum_{|\beta| \geq 2, \beta \leq \alpha} \star\left[\partial^{\beta} \mathbf{A}_{0}^{r} \partial^{\alpha-\beta} \partial_{t} W_{+}+\partial^{\beta} \mathbf{A}_{1}^{r} \partial^{\alpha-\beta} \partial_{x_{1}} W_{+}\right] \\
\quad+\sum_{|\beta| \geq 1, \beta \leq \alpha} \star\left[\partial^{\beta} \mathbf{C}^{r} \partial^{\alpha-\beta} W_{+}\right] .
\end{aligned}
$$

In (38), the $\star$ symbol denotes a constant coefficient that depends on $\alpha$ and $\beta$. There is a similar equation for $\partial^{\alpha} W_{-}$. The difficulty we are facing is that the "zero order" coefficient in (38) (that is, the terms that involve derivatives of order equal to $\ell$ ) does not only involve $\partial^{\alpha} W_{+}$. It also involves the derivatives $\partial^{\alpha-\beta} \partial_{t} W_{+}$and $\partial^{\alpha-\beta} \partial_{x_{1}} W_{+}$, where $|\beta|=1$. These terms cannot be treated in the right-hand side because of the loss of derivatives. We thus write an enlarged system that is satisfied by all the tangential derivatives of order equal to $\ell$. Defining:

$$
W^{(\ell)}:=\left\{\partial_{t}^{\alpha_{0}} \partial_{x_{1}}^{\alpha_{1}} W_{ \pm}, \quad \alpha_{0}+\alpha_{1}=\ell\right\}
$$

the equation (38) and the corresponding one for $\partial^{\alpha} W_{-}$show that $W^{(\ell)}$ satisfies an equation of the form:

$$
\mathcal{A}_{0} \partial_{t} W^{(\ell)}+\mathcal{A}_{1} \partial_{x_{1}} W^{(\ell)}+\mathcal{I} \partial_{x_{2}} W^{(\ell)}+\mathcal{C} W^{(\ell)}=\mathcal{F}^{(\ell)} .
$$

The matrices $\mathcal{A}_{0,1}$ are block diagonal, and their blocks are either $\mathbf{A}_{0,1}^{r}$ or $\mathbf{A}_{0,1}^{l}$. Therefore, they belong to $W^{2, \infty}(\Omega)$. The matrix $\mathcal{C}$ belongs to $W^{1, \infty}(\Omega)$. The matrix $\mathcal{I}$ is block diagonal and its blocks are $\mathbf{I}_{2}$. Eventually, the source term $\mathcal{F}^{(\ell)}$ is a sum of terms that appear in the right-hand side of (38). 
We turn to the boundary conditions satisfied by $W^{(\ell)}$. Starting from (35), we compute:

$$
\begin{aligned}
b \nabla \partial^{\alpha} \psi+b_{\sharp} \partial^{\alpha} \psi & +\mathbf{M} \partial^{\alpha} W_{\left.\right|_{x_{2}=0} ^{n c}}^{n c}=\partial^{\alpha} g \\
& +\sum_{|\beta| \geq 1, \beta \leq \alpha} \star\left[\partial^{\beta} \mathbf{M} \partial^{\alpha-\beta} W_{\left.\right|_{x_{2}=0} ^{n c}}^{n c}+\partial^{\beta} b \nabla \partial^{\alpha-\beta} \psi+\partial^{\beta} b_{\sharp} \partial^{\alpha-\beta} \psi\right] .
\end{aligned}
$$

Following what we have done for the interior equations, the collection of all the equations (40) can be rewritten as:

$$
\mathcal{B} \nabla \psi^{(\ell)}+\mathcal{B}_{\sharp} \psi^{(\ell)}+\mathcal{M} W_{\left.\right|_{x_{2}=0}}^{(\ell), n c}=\mathcal{G}^{(\ell)} .
$$

We claim that the enlarged system (39), (41) satisfies an energy estimate similar to (36). This holds true because the enlarged system satisfies the same regularity and stability properties as the original system (33), (35). We have only collected copies of the original system, and modified the zero order coefficient in the interior equations. However, the a priori estimate in [12], and the well-posedness result of [10] are independent of the zero order coefficient as long as it belongs to $W^{1, \infty}(\Omega)$, which holds true for (39). Therefore we obtain an estimate for the tangential derivatives of order $\ell$ :

$$
\begin{aligned}
\sqrt{\gamma}\left\|W^{(\ell)}\right\|_{L_{\gamma}^{2}\left(\Omega_{T}\right)}+\left\|W_{\mid x_{2}=0}^{(\ell), n c}\right\|_{L_{\gamma}^{2}\left(\omega_{T}\right)} & +\left\|\psi^{(\ell)}\right\|_{H_{\gamma}^{1}\left(\omega_{T}\right)} \\
& \leq C_{\ell}\left(\frac{1}{\gamma^{3 / 2}}\left\|\mathcal{F}^{(\ell)}\right\|_{L^{2}\left(H_{\gamma}^{1}\left(\omega_{T}\right)\right)}+\frac{1}{\gamma}\left\|\mathcal{G}^{(\ell)}\right\|_{H_{\gamma}^{1}\left(\omega_{T}\right)}\right) .
\end{aligned}
$$

We now estimate the source terms $\mathcal{F}^{(\ell)}$ and $\mathcal{G}^{(\ell)}$, that is, the right-hand sides of (38), and (40). The easiest estimates are:

$$
\begin{gathered}
\left\|\partial^{\alpha} F\right\|_{L^{2}\left(H_{\gamma}^{1}\left(\omega_{T}\right)\right)} \simeq \gamma\left\|\partial^{\alpha} F\right\|_{L_{\gamma}^{2}\left(\Omega_{T}\right)}+\left\|\nabla_{t, x_{1}} \partial^{\alpha} F\right\|_{L_{\gamma}^{2}\left(\Omega_{T}\right)} \leq\|F\|_{L^{2}\left(H_{\gamma}^{\ell+1}\left(\omega_{T}\right)\right)}, \\
\left\|\partial^{\alpha} g\right\|_{H_{\gamma}^{1}\left(\omega_{T}\right)} \leq\|g\|_{H_{\gamma}^{\ell+1}\left(\omega_{T}\right)} .
\end{gathered}
$$

Omitting the superscripts $r, l$ or the subscripts \pm , we now turn to the term $\partial^{\beta} \mathbf{A}_{0} \partial_{t} \partial^{\alpha-\beta} W$ in $L^{2}\left(H_{\gamma}^{1}\left(\omega_{T}\right)\right)$, where $\beta \leq \alpha$, and $|\beta| \geq 2$. For a fixed $x_{2}>0$, we apply Gagliardo-Nirenberg's inequality (see Theorems 8 and 10 in Appendix C); then we integrate with respect to $x_{2}$. Decomposing $\beta=\beta^{\prime}+\beta_{1}$, with $\left|\beta_{1}\right|=1$, and recalling that $\partial^{\beta_{1}} \mathbf{A}_{0}$ is a $\mathcal{C}^{\infty}$ function of $\left(\dot{U}, \nabla \dot{\Phi}, \nabla_{t, x_{1}} \dot{U}, \nabla_{t, x_{1}} \nabla \dot{\Phi}\right)$ that vanishes at the origin, we obtain:

$$
\begin{array}{ll}
\left\|\partial^{\beta} \mathbf{A}_{0}\left(x_{2}\right)\right\|_{L_{\gamma}^{p}\left(\omega_{T}\right)} \leq C(K)\left\|\left(\dot{U}, \nabla \dot{\Phi}, \nabla_{t, x_{1}} \dot{U}, \nabla_{t, x_{1}} \nabla \dot{\Phi}\right)\left(x_{2}\right)\right\|_{H_{\gamma}^{\ell-1}\left(\omega_{T}\right)}^{2 / p}, & \frac{2}{p}=\frac{|\beta|-1}{|\alpha|-1}, \\
\left\|\partial^{\alpha-\beta} \partial_{t} W\left(x_{2}\right)\right\|_{L_{\gamma}^{q}\left(\omega_{T}\right)} \leq C\left\|\partial_{t} W\left(x_{2}\right)\right\|_{L^{\infty}\left(\omega_{T}\right)}^{2 / p}\left\|\partial_{t} W\left(x_{2}\right)\right\|_{H_{\gamma}^{\ell-1}\left(\omega_{T}\right)}^{2 / q}, & \frac{2}{q}=\frac{|\alpha|-|\beta|}{|\alpha|-1} .
\end{array}
$$

Using Hölder's inequality and integrating with respect to $x_{2}$, we get:

$$
\begin{aligned}
\left\|\partial^{\beta} \mathbf{A}_{0} \partial_{t} \partial^{\alpha-\beta} W\right\|_{L_{\gamma}^{2}\left(\Omega_{T}\right)} & \\
& \leq C(K)\left\{\|W\|_{L^{2}\left(H_{\gamma}^{\ell}\left(\omega_{T}\right)\right)}+\|W\|_{W^{1, \infty}\left(\Omega_{T}\right)}\|(\dot{U}, \nabla \dot{\Phi})\|_{L^{2}\left(H_{\gamma}^{\ell}\left(\omega_{T}\right)\right)}\right\} .
\end{aligned}
$$


Decomposing $\beta=\beta^{\prime \prime}+\beta_{2}$, with $\left|\beta_{2}\right|=2$, the estimates of the derivatives are similar (recall that we need to estimate source terms in $L^{2}\left(H^{1}\right)$ ):

$$
\begin{aligned}
& \left\|\partial_{t}\left(\partial^{\beta} \mathbf{A}_{0} \partial_{t} \partial^{\alpha-\beta} W\right)\right\|_{L_{\gamma}^{2}\left(\Omega_{T}\right)} \\
& \quad \leq C(K)\left\{\|W\|_{L^{2}\left(H_{\gamma}^{\ell}\left(\omega_{T}\right)\right)}+\|W\|_{W^{1, \infty}\left(\Omega_{T}\right)}\|(\dot{U}, \nabla \dot{\Phi})\|_{L^{2}\left(H_{\gamma}^{\ell+1}\left(\omega_{T}\right)\right)}\right\} \\
& \quad\left\|\partial_{x_{1}}\left(\partial^{\beta} \mathbf{A}_{0} \partial_{t} \partial^{\alpha-\beta} W\right)\right\|_{L_{\gamma}^{2}\left(\Omega_{T}\right)} \\
& \quad \leq C(K)\left\{\|W\|_{L^{2}\left(H_{\gamma}^{\ell}\left(\omega_{T}\right)\right)}+\|W\|_{W^{1, \infty}\left(\Omega_{T}\right)}\|(\dot{U}, \nabla \dot{\Phi})\|_{L^{2}\left(H_{\gamma}^{\ell+1}\left(\omega_{T}\right)\right)}\right\} .
\end{aligned}
$$

We have thus estimated the terms $\partial^{\beta} \mathbf{A}_{0} \partial_{t} \partial^{\alpha-\beta} W$, and the remaining terms $\partial^{\beta} \mathbf{A}_{1} \partial_{x_{1}} \partial^{\alpha-\beta} W, \partial^{\beta} \mathbf{C} \partial^{\alpha-\beta} W$ can be handled in a similar way. These preliminary estimates already enable us to obtain:

$$
\begin{aligned}
& \left\|\mathcal{F}^{(\ell)}\right\|_{L^{2}\left(H_{\gamma}^{1}\left(\omega_{T}\right)\right)} \leq C(K)\left\{\|F\|_{L^{2}\left(H_{\gamma}^{\ell+1}\left(\omega_{T}\right)\right)}\right. \\
& \left.\quad+\gamma\|W\|_{L^{2}\left(H_{\gamma}^{\ell}\left(\omega_{T}\right)\right)}+\|W\|_{W^{1, \infty}\left(\Omega_{T}\right)}\|(\dot{U}, \nabla \dot{\Phi})\|_{H_{\gamma}^{\ell+2}\left(\Omega_{T}\right)}\right\} .
\end{aligned}
$$

The estimate of the right-hand side of (40) is also carried out with the Theorems of Appendix C. We only give the final result:

$$
\begin{aligned}
& \left\|\mathcal{G}^{(\ell)}\right\|_{H_{\gamma}^{1}\left(\omega_{T}\right)} \leq C(K)\left\{\|g\|_{H_{\gamma}^{\ell+1}\left(\omega_{T}\right)}\right. \\
& \quad+\left\|W_{\left.\right|_{x_{2}=0} c}^{n c}\right\|_{H_{\gamma}^{\ell}\left(\omega_{T}\right)}+\|\psi\|_{H_{\gamma}^{\ell+1}\left(\omega_{T}\right)}+\left\|W_{\left.\right|_{x_{2}=0} ^{n c}}^{n}\right\|_{L^{\infty}\left(\omega_{T}\right)}\left\|\left(\dot{U}_{\left.\right|_{x_{2}=0}}, \nabla \varphi\right)\right\|_{H_{\gamma}^{\ell+1}\left(\omega_{T}\right)} \\
& \left.\quad+\|\psi\|_{W^{1, \infty}\left(\omega_{T}\right)}\left\|\left(\dot{U}, \partial_{x_{2}} \dot{U}, \nabla \dot{\Phi}\right)_{\left.\right|_{x_{2}=0}}\right\|_{H_{\gamma}^{\ell+1}\left(\omega_{T}\right)}\right\} .
\end{aligned}
$$

To finish the proof, we use (43), and (44) in (42). Then we multiply this inequality by $\gamma^{m-\ell}$, and sum over $\ell=0, \ldots, m$. Choosing $\gamma$ large enough, we can complete the proof of (37).

\subsection{The vorticity equation}

Since the boundary matrix is singular, all the normal derivatives of $W$ cannot be estimated directly from the equations as in the standard approach for noncharacteristic boundaries, see e.g. [33]. However, some of the normal derivatives, namely those of the "noncharacteristic" part of the solution, can be directly estimated by tangential derivatives. For the Euler equations, it has already been proved by Beirão da Veiga [39], see also [34], that the missing normal derivatives can be estimated through the equation for the vorticity. It is important to note that for our linearized problem (22), (25), there is no loss of derivatives when estimating the normal derivatives (see, e.g., [26, 1, 16, 35, 36] for the general case where losses of normal derivatives occur and where Sobolev spaces with conormal regularity are required).

In order to introduce the linearized vorticity equation, let us consider first of all the original Euler system (1). If there exists a solution that is smooth on either side of an interface, this solution satisfies

$$
\rho\left(\partial_{t} \mathbf{u}+\left(\mathbf{u} \cdot \nabla_{x}\right) \mathbf{u}\right)+\nabla_{x} p(\rho)=0, \quad \mathbf{u}=\left(\begin{array}{l}
v \\
u
\end{array}\right) .
$$

Hence the (scalar) vorticity $\xi:=\partial_{x_{1}} u-\partial_{x_{2}} v$ satisfies (on either side of the interface):

$$
\partial_{t} \xi+\mathbf{u} \cdot \nabla_{x} \xi+\xi\left(\nabla_{x} \cdot \mathbf{u}\right)=0 .
$$

$4^{\mathrm{e}}$ SÉRIE - TOME $41-2008-\mathrm{N}^{\mathrm{o}} 1$ 
Recalling that the interface is a streamline, and that there is continuity of the normal velocity across the interface, this suggests the possibility of estimating the vorticity on either part of the front. Performing the change of variables by the introduction of the functions $\Phi^{ \pm}$and taking account of the linearization leads to the definition of the following "linearized vorticity":

$$
\dot{\xi}:=\partial_{x_{1}} \dot{u}-\frac{1}{\partial_{x_{2}} \Phi}\left(\partial_{x_{1}} \Phi \partial_{x_{2}} \dot{u}+\partial_{x_{2}} \dot{v}\right)
$$

where we have omitted the subscripts $\pm, r, l$. Introducing the (scalar) source terms:

$$
\mathcal{F}_{1}^{ \pm}:=\left(f_{ \pm}-C\left(U_{r, l}, \Phi_{r, l}\right) \dot{V}_{ \pm}\right)_{2}, \quad \mathcal{F}_{2}^{ \pm}:=\left(f_{ \pm}-C\left(U_{r, l}, \Phi_{r, l}\right) \dot{V}_{ \pm}\right)_{3}
$$

the equations for $\dot{\xi}_{ \pm}$are:

$$
\begin{gathered}
\partial_{t} \dot{\xi}_{+}+v_{r} \partial_{x_{1}} \dot{\xi}_{+}=\partial_{x_{1}} \mathcal{F}_{2}^{+}-\frac{1}{\partial_{x_{2}} \Phi_{r}}\left(\partial_{x_{1}} \Phi_{r} \partial_{x_{2}} \mathcal{F}_{2}^{+}+\partial_{x_{2}} \mathcal{F}_{1}^{+}\right)+\Lambda_{1}^{r} \cdot \partial_{x_{1}} \dot{V}_{+}+\Lambda_{2}^{r} \cdot \partial_{x_{2}} \dot{V}_{+} \\
\partial_{t} \dot{\xi}_{-}+v_{l} \partial_{x_{1}} \dot{\xi}_{-}=\partial_{x_{1}} \mathcal{F}_{2}^{-}-\frac{1}{\partial_{x_{2}} \Phi_{l}}\left(\partial_{x_{1}} \Phi_{l} \partial_{x_{2}} \mathcal{F}_{2}^{-}+\partial_{x_{2}} \mathcal{F}_{1}^{-}\right)+\Lambda_{1}^{l} \cdot \partial_{x_{1}} \dot{V}_{-}+\Lambda_{2}^{l} \cdot \partial_{x_{2}} \dot{V}_{-}
\end{gathered}
$$

In (47), the vectors $\Lambda_{1}^{r, l}, \Lambda_{2}^{r, l}$ are $\mathcal{C}^{\infty}$ functions of $\left(\dot{U}_{r, l}, \nabla \dot{U}_{r, l}, \nabla \dot{\Phi}_{r, l}, \nabla^{2} \dot{\Phi}_{r, l}\right)$ that vanish at the origin, and whose exact expression is of no interest. The estimates of $\dot{\xi}_{ \pm}$are as follows:

Proposition 2. - Let $m \in \mathbb{N}, m \geq 1$, and $T>0$. There exist two constants $C_{m}>0$ and $\gamma_{m} \geq 1$, that do not depend on $T$, such that for all $\gamma \geq \gamma_{m}$, the smooth solutions $\dot{\xi}_{ \pm}$to (47) satisfy the following a priori estimate:

$$
\begin{array}{r}
\sqrt{\gamma}\left\|\dot{\xi}_{ \pm}\right\|_{H_{\gamma}^{m-1}\left(\Omega_{T}\right)} \leq \frac{C_{m}}{\sqrt{\gamma}}\{\| \\
\left\|f_{ \pm}\right\|_{H_{\gamma}^{m}\left(\Omega_{T}\right)}+\left\|\dot{V}_{ \pm}\right\|_{H_{\gamma}^{m}\left(\Omega_{T}\right)}+\left\|f_{ \pm}\right\|_{L^{\infty}\left(\Omega_{T}\right)}\left\|\nabla \dot{\Phi}_{r, l}\right\|_{H_{\gamma}^{m}\left(\Omega_{T}\right)} \\
\left.+\left\|\dot{V}_{ \pm}\right\|_{W^{1, \infty}\left(\Omega_{T}\right)}\left(\left\|\dot{U}_{r, l}\right\|_{H^{m+1}\left(\Omega_{T}\right)}+\left\|\nabla \dot{\Phi}_{r, l}\right\|_{H^{m}\left(\Omega_{T}\right)}\right)\right\} .
\end{array}
$$

The proof of Proposition 2 is a straightforward application of the energy method for the transport equations (47). The estimate of the source terms in $H_{\gamma}^{m-1}\left(\Omega_{T}\right)$ relies on the Gagliardo-Nirenberg inequalities. Since we have already detailed such estimates in the preceding paragraph, we skip the details here, and now turn to the estimates of the other normal derivatives.

\subsection{A priori estimate of normal derivatives}

From the exact definition of the matrix $T(U, \nabla \Phi)$ in [12], we can compute the relations:

$$
\begin{aligned}
& \partial_{x_{2}} \dot{v}_{+}=\partial_{x_{2}} W_{+, 1}-\frac{c\left(\rho_{r}\right)}{\rho_{r}} \partial_{x_{1}} \Phi_{r}\left(\partial_{x_{2}} W_{+, 2}-\partial_{x_{2}} W_{+, 3}\right)+\left(\left[\partial_{x_{2}} T\left(U_{r}, \Phi_{r}\right)^{-1}\right] W_{+}\right)_{2}, \\
& \partial_{x_{2}} \dot{u}_{+}=\partial_{x_{1}} \Phi_{r} \partial_{x_{2}} W_{+, 1}+\frac{c\left(\rho_{r}\right)}{\rho_{r}}\left(\partial_{x_{2}} W_{+, 2}-\partial_{x_{2}} W_{+, 3}\right)+\left(\left[\partial_{x_{2}} T\left(U_{r}, \Phi_{r}\right)\right]^{-1} W_{+}\right)_{3} .
\end{aligned}
$$

Substituting into the definition (45) of the vorticity $\dot{\xi}_{+}$gives:

$$
\partial_{x_{2}} W_{+, 1}=\frac{1}{\left\langle\partial_{x_{1}} \Phi_{r}\right\rangle^{2}}\left\{\partial_{x_{2}} \Phi_{r}\left(\partial_{x_{1}} \dot{u}_{+}-\dot{\xi}_{+}\right)-\partial_{x_{1}} \Phi_{r}\left(\partial_{x_{2}} T_{r}^{-1} W_{+}\right)_{3}-\left(\partial_{x_{2}} T_{r}^{-1} W_{+}\right)_{2}\right\}
$$


Consequently the normal derivative $\partial_{x_{2}} W_{+, 1}$ may be expressed, up to lower order terms, as a linear combination of tangential derivatives of $W_{+}$and the vorticity $\dot{\xi}_{+}$. As regards the remaining normal derivatives $\partial_{x_{2}} W_{+, 2}$, and $\partial_{x_{2}} W_{+, 3}$, they are directly given by (33):

$$
\mathbf{I}_{2} \partial_{x_{2}} W_{+}=\mathbf{I}_{2}\left(F_{+}-\mathbf{A}_{0}^{r} \partial_{t} W_{+}-\mathbf{A}_{1}^{r} \partial_{x_{1}} W_{+}-\mathbf{C}^{r} W_{+}\right) .
$$

We recall that $\mathbf{I}_{2}$ is defined by (31). We may combine this latter relation with (49), and write:

$$
\partial_{x_{2}} W_{+}=\mathbf{I}_{2} F_{+}+\widetilde{\mathbf{A}}_{0}^{r} \partial_{t} W_{+}+\widetilde{\mathbf{A}}_{1}^{r} \partial_{x_{1}} W_{+}+\widetilde{\mathbf{C}}^{r} W_{+}-\frac{\partial_{x_{2}} \Phi_{r}}{\left\langle\partial_{x_{1}} \Phi_{r}\right\rangle^{2}}\left(\begin{array}{c}
\dot{\xi}_{+} \\
0 \\
0
\end{array}\right),
$$

with new matrices $\widetilde{\mathbf{A}}_{0,1}^{r}$, and $\widetilde{\mathbf{C}}^{r}$. The matrices $\widetilde{\mathbf{A}}_{0,1}^{r}$ are $\mathcal{C}^{\infty}$ functions of their arguments $\left(\dot{U}_{r}, \nabla \dot{\Phi}_{r}\right)$, and $\widetilde{\mathbf{C}}^{r}$ is a $\mathcal{C}^{\infty}$ function of its arguments $\left(\dot{U}_{r}, \nabla \dot{U}_{r}, \nabla \dot{\Phi}_{r}, \nabla^{2} \dot{\Phi}_{r}\right)$. These matrices include the contribution of the term $\partial_{x_{1}} \dot{u}_{+}$and the contribution of the zero order terms in (49). There is a similar equation for $\partial_{x_{2}} W_{-}$.

The equation (50) shows that, though the linearized problem (33), (35) is characteristic, all the normal derivatives are given, up to lower order terms, as a linear combination of tangential derivatives and the vorticity. This allows to prove the following result:

Proposition 3. - Let $m \in \mathbb{N}, m \geq 1$, and let $T>0$. Then there exist some constants $C_{m}$ and $\gamma_{m}$, that do not depend on $T$, such that the following a priori estimate holds for all $\gamma \geq \gamma_{m}$, and all $k=1, \ldots, m$ :

$$
\begin{aligned}
\| \partial_{x_{2}}^{k} & W_{ \pm} \|_{L^{2}\left(H_{\gamma}^{m-k}\left(\omega_{T}\right)\right)} \leq C_{m}\left\{\left\|F_{ \pm}\right\|_{H_{\gamma}^{m-1}\left(\Omega_{T}\right)}+\left\|\dot{\xi}_{ \pm}\right\|_{H_{\gamma}^{m-1}\left(\Omega_{T}\right)}\right. \\
& +\left\|\dot{\xi}_{ \pm}\right\|_{L^{\infty}\left(\Omega_{T}\right)}\left\|\nabla \dot{\Phi}_{r, l}\right\|_{H_{\gamma}^{m-1}\left(\Omega_{T}\right)}+\left\|W_{ \pm}\right\|_{L^{2}\left(H_{\gamma}^{m}\left(\omega_{T}\right)\right)}+\left\|W_{ \pm}\right\|_{H_{\gamma}^{m-1}\left(\Omega_{T}\right)} \\
& \left.+\left\|W_{ \pm}\right\|_{L^{\infty}\left(\Omega_{T}\right)}\left\|\left(\dot{U}_{r, l}, \nabla \dot{\Phi}_{r, l}\right)\right\|_{H_{\gamma}^{m}\left(\Omega_{T}\right)}\right\} .
\end{aligned}
$$

Proof. - The proof follows from an induction argument. We only give the proof for $W_{+}$. For $k=1$, we use (50). Using Theorem 9 of Appendix $C$ for a fixed $x_{2}$ and integrating with respect to $x_{2}$, we get:

$$
\begin{aligned}
\left\|\mathbf{I}_{2} F_{+}\right\|_{L^{2}\left(H_{\gamma}^{m-1}\left(\omega_{T}\right)\right)} \leq & C\left\|F_{+}\right\|_{H_{\gamma}^{m-1}\left(\Omega_{T}\right)}, \\
\left\|\widetilde{\mathbf{C}}^{r} W_{+}\right\|_{L^{2}\left(H_{\gamma}^{m-1}\left(\omega_{T}\right)\right)} \leq & C(K)\left\{\left\|W_{+}\right\|_{L^{2}\left(H_{\gamma}^{m-1}\left(\omega_{T}\right)\right)}\right. \\
& \left.+\left\|W_{+}\right\|_{L^{\infty}\left(\Omega_{T}\right)}\left\|\left(\dot{U}_{r}, \nabla \dot{\Phi}_{r}\right)\right\|_{L^{2}\left(H_{\gamma}^{m}\left(\omega_{T}\right)\right)}\right\} .
\end{aligned}
$$

Decomposing:

$$
\frac{\partial_{x_{2}} \Phi_{r}}{\left\langle\partial_{x_{1}} \Phi_{r}\right\rangle^{2}} \dot{\xi}_{+}=\dot{\xi}_{+}+H\left(\nabla \dot{\Phi}_{r}\right) \dot{\xi}_{+}
$$

where $H$ is a $\mathcal{C}^{\infty}$ function that vanishes at the origin, we also get:

$$
\begin{aligned}
&\left\|\frac{\partial_{x_{2}} \Phi_{r}}{\left\langle\partial_{x_{1}} \Phi_{r}\right\rangle^{2}} \dot{\xi}_{+}\right\|_{L^{2}\left(H_{\gamma}^{m-1}\left(\omega_{T}\right)\right)} \\
& \quad \leq C(K)\left\{\left\|\dot{\xi}_{+}\right\|_{L^{2}\left(H_{\gamma}^{m-1}\left(\omega_{T}\right)\right)}+\left\|\dot{\xi}_{+}\right\|_{L^{\infty}\left(\Omega_{T}\right)}\left\|\nabla \dot{\Phi}_{r}\right\|_{L^{2}\left(H_{\gamma}^{m-1}\left(\omega_{T}\right)\right)}\right\} .
\end{aligned}
$$

$4^{\text {e }}$ SÉRIE - TOME $41-2008-\mathrm{N}^{\mathrm{o}} 1$ 
We now turn to the estimate of $\widetilde{\mathbf{A}}_{0}^{r} \partial_{t} W_{+}$. Applying a tangential derivative $\partial^{\alpha}=\partial_{t}^{\alpha_{0}} \partial_{x_{1}}^{\alpha_{1}}$ to this product, where $\ell=|\alpha| \leq m-1$, we get:

$$
\partial^{\alpha}\left[\widetilde{\mathbf{A}}_{0}^{r} \partial_{t} W_{+}\right]=\widetilde{\mathbf{A}}_{0}^{r} \partial_{t} \partial^{\alpha} W_{+}+\sum_{|\beta| \geq 1, \beta \leq \alpha} \star\left[\partial^{\beta} \widetilde{\mathbf{A}}_{0}^{r} \partial^{\alpha-\beta} \partial_{t} W_{+}\right] .
$$

The first term is easily estimated in $L_{\gamma}^{2}\left(\Omega_{T}\right)$, while all the terms in the sum are estimated thanks to Gagliardo-Nirenberg's inequality (Theorem 8), as for the estimates of the tangential derivatives of $W_{+}$. We obtain:

$$
\begin{aligned}
& \left\|\partial^{\alpha}\left[\widetilde{\mathbf{A}}_{0}^{r} \partial_{t} W_{+}\right]\right\|_{L_{\gamma}^{2}\left(\Omega_{T}\right)} \\
& \quad \leq C(K)\left\{\left\|W_{+}\right\|_{L^{2}\left(H_{\gamma}^{\ell+1}\left(\omega_{T}\right)\right)}+\left\|W_{+}\right\|_{L^{\infty}\left(\Omega_{T}\right)}\left\|\left(\dot{U}_{r}, \nabla \dot{\Phi}_{r}\right)\right\|_{L^{2}\left(H_{\gamma}^{\ell+1}\left(\omega_{T}\right)\right)}\right\} .
\end{aligned}
$$

Multiplying by $\gamma^{m-1-\ell}$, and summing over $\ell=0, \ldots, m-1$, we get:

$$
\begin{aligned}
& \left\|\widetilde{\mathbf{A}}_{0}^{r} \partial_{t} W_{+}\right\|_{L^{2}\left(H_{\gamma}^{m-1}\left(\omega_{T}\right)\right)} \\
& \quad \leq C(K)\left\{\left\|W_{+}\right\|_{L^{2}\left(H_{\gamma}^{m}\left(\omega_{T}\right)\right)}+\left\|W_{+}\right\|_{L^{\infty}\left(\Omega_{T}\right)}\left\|\left(\dot{U}_{r}, \nabla \dot{\Phi}_{r}\right)\right\|_{L^{2}\left(H_{\gamma}^{m}\left(\omega_{T}\right)\right)}\right\} .
\end{aligned}
$$

A similar estimate holds for $\widetilde{\mathbf{A}}_{1}^{r} \partial_{x_{1}} W_{+}$. This completes the proof of (51) in the case $k=1$. To close the induction argument, we assume that (51) holds up to some integer $k$, then we apply $\partial_{x_{2}}^{k}$ to (50). Developing all the derivatives by Leibniz' formula, we estimate each term separately by the Gagliardo-Nirenberg's inequality (first for fixed $x_{2}$, then integrate with respect to $x_{2}$ ). We omit the details that are very similar to what we have already done.

\subsection{The a priori tame estimate}

The results of the preceding paragraphs enable us to prove the following tame estimate in the $H_{\gamma}^{m}$ norm:

Theorem 4. - Let $m \in \mathbb{N}$, and let $T>0$. Assume that the stationary solution(11) satisfies (12). Assume also that the perturbations $\dot{U}, \dot{\Phi}$ in (14) satisfy (15), (16), (17), (26), and:

$$
\forall \gamma \geq 1, \quad\left(\dot{U}_{r}, \dot{U}_{l}, \dot{\Phi}_{r}, \dot{\Phi}_{l}\right) \in H_{\gamma}^{m+3}\left(\Omega_{T}\right) .
$$

Then there exists a constant $K_{0}>0$, that does not depend on $m$ and $T$, and there exist two constants $C_{m}>0$ and $\gamma_{m} \geq 1$, that depend on $m$ but not on $T$, such that, if $K \leq K_{0}, \gamma \geq \gamma_{m}$, and if $\left(\dot{V}_{ \pm}, \psi\right) \in H_{\gamma}^{m+2}\left(\Omega_{T}\right) \times H_{\gamma}^{m+2}\left(\omega_{T}\right)$ is a solution to (22), (25), then one has:

$$
\begin{aligned}
\sqrt{\gamma} & \|\dot{V}\|_{H_{\gamma}^{m}\left(\Omega_{T}\right)}+\left\|\mathbb{P}(\varphi) \dot{V}_{\left.\right|_{x_{2}=0}}\right\|_{H_{\gamma}^{m}\left(\omega_{T}\right)}+\|\psi\|_{H_{\gamma}^{m+1}\left(\omega_{T}\right)} \\
\leq & C_{m}\left\{\frac{1}{\sqrt{\gamma}}\|f\|_{H_{\gamma}^{m}\left(\Omega_{T}\right)}+\frac{1}{\gamma^{3 / 2}}\|f\|_{L^{2}\left(H_{\gamma}^{m+1}\left(\omega_{T}\right)\right)}+\frac{1}{\gamma}\|g\|_{H_{\gamma}^{m+1}\left(\omega_{T}\right)}\right. \\
& +\frac{1}{\gamma^{3 / 2}}\|f\|_{L^{\infty}\left(\Omega_{T}\right)}\|(\dot{U}, \nabla \dot{\Phi})\|_{H_{\gamma}^{m+1}\left(\Omega_{T}\right)}+\frac{1}{\gamma^{3 / 2}}\|\dot{V}\|_{W^{1, \infty}\left(\Omega_{T}\right)}\|(\dot{U}, \nabla \dot{\Phi})\|_{H_{\gamma}^{m+2}\left(\Omega_{T}\right)} \\
& \left.+\frac{1}{\gamma}\left(\left\|\mathbb{P}(\varphi) \dot{V}_{x_{2}=0}\right\|_{L^{\infty}\left(\omega_{T}\right)}+\|\psi\|_{W^{1, \infty}\left(\omega_{T}\right)}\right)\left\|\left(\dot{U}, \partial_{x_{2}} \dot{U}, \nabla \dot{\Phi}\right)_{\left.\right|_{x_{2}=0}}\right\|_{H_{\gamma}^{m+1}\left(\omega_{T}\right)}\right\} .
\end{aligned}
$$


The proof consists in first obtaining a tame estimate for $W$ with respect to the source terms $F$, and $g$ in (33), (35). This estimate follows from (37) and (51), and by choosing $\gamma$ large. Then we recover (52) by using the definitions (32) and (34). Once again, the estimates of products derive from the results in Appendix C.

REMARK 5. - The estimate (52) is not exactly a tame estimate in the usual sense, since the solution $(\dot{V}, \psi)$ appears in the right-hand side through $L^{\infty}$ or $W^{1, \infty}$ norms. However, these norms can be absorbed in the left-hand side of the inequality thanks to the classical Sobolev imbedding Theorem (provided that $\gamma$ and $T$ are fixed), see Proposition 6 below.

The estimate (52) is an a priori estimate, in the sense that it only gives an information for smooth solutions. However, we already know that the linearized problem (22), (25) is well-posed for source terms $(f, g)$ in $L^{2}\left(H^{1}\left(\omega_{T}\right)\right) \times H^{1}\left(\omega_{T}\right)$ that vanish in the past. Following [33, 7], Theorem 4 can be converted into a well-posedness result for source terms $(f, g)$ in $H^{m+1}\left(\Omega_{T}\right) \times$ $H^{m+1}\left(\omega_{T}\right)$ that vanish in the past. The corresponding solution $(\dot{V}, \psi)$ belongs to $H^{m}\left(\Omega_{T}\right) \times$ $H^{m+1}\left(\omega_{T}\right)$, vanishes in the past, the trace $\mathbb{P}(\varphi) \dot{V}_{\left.\right|_{x_{2}=0}}$ belongs to $H^{m}\left(\omega_{T}\right)$, and (52) is satisfied for all $\gamma \geq \gamma_{m}$.

\section{Compatibility conditions for the initial data}

\subsection{The compatibility conditions}

Let $k \in \mathbb{N}$, with $k \geq 3$. Given initial data $U_{0}^{ \pm}=\left(\rho_{0}^{ \pm}, v_{0}^{ \pm}, u_{0}^{ \pm}\right)$such that $U_{0}^{ \pm}=\bar{U}^{ \pm}+\dot{U}_{0}^{ \pm}$, where $\dot{U}_{0}^{ \pm} \in H^{k+1 / 2}\left(\mathbb{R}_{+}^{2}\right)$, and $\varphi_{0} \in H^{k+1}(\mathbb{R})$, we need to prescribe the necessary compatibility conditions for the existence of a smooth solution $\left(U^{ \pm}, \Phi^{ \pm}\right)$to (3), (4), (5), (6), and (7). As we will see, the choice of the functions $\Phi^{ \pm}$, which are required to satisfy (7), and (4a) makes the formulation of the compatibility conditions rather simple. We assume that the initial data $\dot{U}_{0}^{ \pm}$and $\varphi_{0}$ have compact support:

$$
\operatorname{Supp} \dot{U}_{0}^{ \pm} \subset\left\{x_{2} \geq 0, \sqrt{x_{1}^{2}+x_{2}^{2}} \leq 1\right\}, \quad \operatorname{Supp} \varphi_{0} \subset[-1,1] .
$$

Let us first extend $\varphi_{0}$ to $\mathbb{R}_{+}^{2}=\mathbb{R}_{x_{1}} \times \mathbb{R}_{x_{2}}^{+}$by constructing $\dot{\Phi}_{0}^{+}=\dot{\Phi}_{0}^{-} \in H^{k+3 / 2}\left(\mathbb{R}_{+}^{2}\right)$, that satisfy $\left(\dot{\Phi}_{0}^{ \pm}\right)_{\mid x_{2}=0}=\varphi_{0}$ and the estimate:

$$
\left\|\dot{\Phi}_{0}^{ \pm}\right\|_{H^{k+3 / 2}\left(\mathbb{R}_{+}^{2}\right)} \leq C\left\|\varphi_{0}\right\|_{H^{k+1}(\mathbb{R})} .
$$

Up to multiplying $\dot{\Phi}_{0}^{ \pm}$by a $\mathcal{C}^{\infty}$ function with compact support (whose choice only depends on the support of $\varphi_{0}$ ), we may assume that $\dot{\Phi}_{0}^{ \pm}$satisfy:

$$
\text { Supp } \dot{\Phi}_{0}^{ \pm} \subset\left\{x_{2} \geq 0, \sqrt{x_{1}^{2}+x_{2}^{2}} \leq 2\right\} .
$$

We define $\Phi_{0}^{ \pm}:= \pm x_{2}+\dot{\Phi}_{0}^{ \pm}$. Because $k+3 / 2>3$, the Sobolev's imbedding Theorem yields:

$$
\forall x \in \mathbb{R}_{+}^{2}, \quad \partial_{x_{2}} \Phi_{0}^{+}(x) \geq \frac{7}{8}, \quad \partial_{x_{2}} \Phi_{0}^{-}(x) \leq-\frac{7}{8},
$$

provided that $\varphi_{0}$ is sufficiently small in $H^{k+1}(\mathbb{R})$, see (54).

Let us prescribe, for the eikonal equations (7) that must be satisfied by $\Phi^{ \pm}$, the initial data:

$$
\Phi_{\mid t=0}^{ \pm}=\Phi_{0}^{ \pm},
$$

in the space domain $\mathbb{R}_{+}^{2}$. Of course, we look for a solution $\Phi^{ \pm}$of the form $\Phi^{ \pm}= \pm x_{2}+\dot{\Phi}^{ \pm}$. 
To derive the compatibility conditions, we follow the approach of [33]. We first note that the equations (3) and (7), when evaluated at time $t=0$, determine the traces $\partial_{t} \dot{U}_{\mid t=0}^{ \pm}$and $\partial_{t} \dot{\Phi}_{\mid t=0}^{ \pm}$in terms of the initial data $\dot{U}_{0}^{ \pm}$and $\dot{\Phi}_{0}^{ \pm}$:

$$
\left\{\begin{aligned}
\partial_{t} \dot{\Phi}_{\mid t=0}^{ \pm}= & -v_{0}^{ \pm} \partial_{x_{1}} \dot{\Phi}_{0}^{ \pm}+\dot{u}_{0}^{ \pm} \\
\partial_{t} \dot{U}_{\mid t=0}^{ \pm}= & -A_{1}\left(U_{0}^{ \pm}\right) \partial_{x_{1}} \dot{U}_{0}^{ \pm} \\
& -\frac{1}{\partial_{x_{2}} \Phi_{0}^{ \pm}}\left(A_{2}\left(U_{0}^{ \pm}\right)+v_{0}^{ \pm} \partial_{x_{1}} \dot{\Phi}_{0}^{ \pm}-\dot{u}_{0}^{ \pm}-\partial_{x_{1}} \dot{\Phi}_{0}^{ \pm} A_{1}\left(U_{0}^{ \pm}\right)\right) \partial_{x_{2}} \dot{U}_{0}^{ \pm}
\end{aligned}\right.
$$

These expressions can be generalized to higher order derivatives. Taking formally $j$ time derivatives of (3) and (7) determines inductively the $(j+1)$-th order derivatives $\partial_{t}^{j+1} \dot{U}^{ \pm}$, $\partial_{t}^{j+1} \dot{\Phi}^{ \pm}$as functions of $\dot{U}^{ \pm}, \dot{\Phi}^{ \pm}$, and of their space derivatives up to order $(j+1)$. More precisely, let us denote the traces at time $t=0$ by $\dot{U}_{\ell}^{ \pm}:=\partial_{t}^{\ell} \dot{U}_{\mid t=0}^{ \pm}$and $\dot{\Phi}_{\ell}^{ \pm}:=\partial_{t}^{\ell} \dot{\Phi}_{\mid t=0}^{ \pm}$for all integer $\ell$. For $j \geq 1$, Leibniz' rule yields the expression:

$$
\dot{\Phi}_{j+1}^{ \pm}=\dot{u}_{j}^{ \pm} \mp \bar{v} \partial_{x_{1}} \dot{\Phi}_{j}^{ \pm}-\sum_{\ell=0}^{j} C_{j}^{\ell} \dot{v}_{\ell}^{ \pm} \partial_{x_{1}} \dot{\Phi}_{j-\ell}^{ \pm} .
$$

Moreover, if we denote $\mathcal{W}^{ \pm}:=\left(\dot{U}^{ \pm}, \nabla_{x} \dot{U}^{ \pm}, \nabla_{x} \dot{\Phi}^{ \pm}\right)$, we can rewrite (3) under the form:

$$
\partial_{t} \dot{U}^{ \pm}=\mathbf{F}\left(\mathcal{W}^{ \pm}\right)
$$

where $\mathbf{F}$ is a suitable $\mathcal{C}^{\infty}$ function that vanishes at the origin. We take $j-1$ time derivatives of this expression, then we take the trace at time $t=0$. The Faà di Bruno's formula yields:

$$
\dot{U}_{j+1}^{ \pm}=\sum_{\alpha \in \mathbb{N}^{j}, \alpha_{1}+\cdots+j \alpha_{j}=j} \frac{j !}{\alpha_{1} ! \ldots \alpha_{j} !} D^{|\alpha|} \mathbf{F}\left(\mathcal{W}_{0}\right)\left\{\left(\frac{\mathcal{W}_{1}}{1 !}\right)^{\otimes^{\alpha_{1}}}, \ldots,\left(\frac{\mathcal{W}_{j}}{j !}\right)^{\otimes^{\alpha_{j}}}\right\}
$$

where we have used the obvious notation $\mathcal{W}_{\ell}^{ \pm}=\left(\dot{U}_{\ell}^{ \pm}, \nabla_{x} \dot{U}_{\ell}^{ \pm}, \nabla_{x} \dot{\Phi}_{\ell}^{ \pm}\right)$. It is clear that the relations (58), (59) and (60) form an induction relation for the sequence $\left(\dot{U}_{j}^{ \pm}, \dot{\Phi}_{j}^{ \pm}\right)_{j \geq 0}$ that enables us to determine $\dot{U}_{j}^{ \pm}$and $\dot{\Phi}_{j}^{ \pm}$in terms of the initial data $\dot{U}_{0}^{ \pm}$and $\dot{\Phi}_{0}^{ \pm}$.

With the help of (58), (59) and (60), we can prove the following result by following exactly the proof of [29, Lemma 4.2.1]:

Lemma 2. - Let $k \in \mathbb{N}, k \geq 3$. Let $U_{0}^{ \pm}=\bar{U}^{ \pm}+\dot{U}_{0}^{ \pm}, \dot{U}_{0}^{ \pm} \in H^{k+1 / 2}\left(\mathbb{R}_{+}^{2}\right)$, and let $\varphi_{0} \in H^{k+1}(\mathbb{R})$, where $\dot{U}_{0}^{ \pm}$and $\varphi_{0}$ satisfy (53). Consider the functions $\Phi_{0}^{ \pm}= \pm x_{2}+\dot{\Phi}_{0}^{ \pm}$ that we have constructed above, and that satisfy (54), (55) and (56) if $\varphi_{0}$ is sufficiently small. Then the equations (59) and (60) determine $\dot{U}_{j}^{ \pm} \in H^{k+1 / 2-j}\left(\mathbb{R}_{+}^{2}\right)$, for $j=1, \ldots, k$ and $\dot{\Phi}_{\ell}^{ \pm} \in$ $H^{k+3 / 2-\ell}\left(\mathbb{R}_{+}^{2}\right)$, for $\ell=1, \ldots, k+1$. Moreover, these functions satisfy:

$$
\operatorname{Supp} \dot{\Phi}_{j}^{ \pm} \subset\left\{x_{2} \geq 0, \sqrt{x_{1}^{2}+x_{2}^{2}} \leq 2\right\}, \quad \operatorname{Supp} \dot{U}_{j}^{ \pm} \subset\left\{x_{2} \geq 0, \sqrt{x_{1}^{2}+x_{2}^{2}} \leq 1\right\},
$$

and there exists a constant $C>0$, that only depends on $k$ and $\left\|\left(\dot{U}_{0}^{ \pm}, \dot{\Phi}_{0}^{ \pm}\right)\right\|_{W^{1, \infty}\left(\mathbb{R}_{+}^{2}\right)}$, such that: (61)

$$
\sum_{j=1}^{k}\left\|\dot{U}_{j}^{ \pm}\right\|_{H^{k+1 / 2-j}\left(\mathbb{R}_{+}^{2}\right)}+\sum_{\ell=1}^{k+1}\left\|\dot{\Phi}_{\ell}^{ \pm}\right\|_{H^{k+3 / 2-\ell}\left(\mathbb{R}_{+}^{2}\right)} \leq C\left(\left\|\dot{U}_{0}^{ \pm}\right\|_{H^{k+1 / 2}\left(\mathbb{R}_{+}^{2}\right)}+\left\|\varphi_{0}\right\|_{H^{k+1}(\mathbb{R})}\right) .
$$

We refer to [33] and [29, Lemma 4.2.1] for the proof (the proof in [33] is for $\mathcal{C}^{\infty}$ coefficients). We now introduce the following terminology: 
Definition 1. - Let $k \geq 3$. Let $U_{0}^{ \pm}=\left(\rho_{0}^{ \pm}, v_{0}^{ \pm}, u_{0}^{ \pm}\right)$be such that $U_{0}^{ \pm}=\bar{U}^{ \pm}+\dot{U}_{0}^{ \pm}$, with $\dot{U}_{0}^{ \pm} \in H^{k+1 / 2}\left(\mathbb{R}_{+}^{2}\right)$, let $\varphi_{0} \in H^{k+1}(\mathbb{R})$, that satisfy (53). Consider the functions $\Phi_{0}^{ \pm}= \pm x_{2}+$ $\dot{\Phi}_{0}^{ \pm}$that we have constructed above and that satisfy (54), (55) and (56), when $\varphi_{0}$ is sufficiently small.

The initial data $\left(\dot{U}_{0}^{ \pm}, \varphi_{0}\right)$ are said to be compatible up to order $k$ if the traces of the functions $\dot{U}_{1}^{ \pm}, \ldots, \dot{U}_{k}^{ \pm}, \dot{\Phi}_{1}^{ \pm}, \ldots, \dot{\Phi}_{k+1}^{ \pm}$satisfy:

$$
\begin{array}{ll}
\partial_{x_{2}}^{\ell}\left(\dot{\Phi}_{j}^{+}-\dot{\Phi}_{j}^{-}\right)_{\mid x_{2}=0}=0, & \text { for } j=0, \ldots, k, \text { and } \ell=0, \ldots, k-j, \\
\partial_{x_{2}}^{\ell}\left(\dot{\rho}_{j}^{+}-\dot{\rho}_{j}^{-}\right)_{\mid x_{2}=0}=0, & \text { for } j=0, \ldots, k-1, \text { and } \ell=0, \ldots, k-1-j,
\end{array}
$$

and

$$
\begin{array}{ll}
\int_{\mathbb{R}_{+}^{2}}\left|\partial_{x_{2}}^{k+1-j}\left(\dot{\Phi}_{j}^{+}-\dot{\Phi}_{j}^{-}\right)\right|^{2} d x_{1} \frac{d x_{2}}{x_{2}}<+\infty, & \text { for } j=0, \ldots, k+1, \\
\int_{\mathbb{R}_{+}^{2}}\left|\partial_{x_{2}}^{k-j}\left(\dot{\rho}_{j}^{+}-\dot{\rho}_{j}^{-}\right)\right|^{2} d x_{1} \frac{d x_{2}}{x_{2}}<+\infty, & \text { for } j=0, \ldots, k .
\end{array}
$$

Observe that $\dot{\rho}_{0}^{ \pm}, \ldots, \dot{\rho}_{k-1}^{ \pm}, \dot{\Phi}_{0}^{ \pm}, \ldots, \dot{\Phi}_{k}^{ \pm} \in H^{3 / 2}\left(\mathbb{R}_{+}^{2}\right)$, so it is legitimate to consider the traces of these functions on $\left\{x_{2}=0\right\}$. The second set of equalities in (62) obviously follows from (4d), since it requires the equality of time derivatives of $\rho^{ \pm}=\bar{\rho}+\dot{\rho}^{ \pm}$. The first set of equalities follows as well from (4a). We note that these equalities automatically yield the compatibility conditions for the last two boundary conditions (4b) and (4c). Namely, an equivalent formulation of (4b) and (4c) is:

$$
\partial_{t} \varphi+v_{\left.\right|_{x_{2}}=0}^{+} \partial_{x_{1}} \varphi-u_{\left.\right|_{x_{2}=0}}^{+}=\partial_{t} \varphi+v_{\left.\right|_{x_{2}}=0}^{-} \partial_{x_{1}} \varphi-u_{\left.\right|_{x_{2}=0}}^{-}=0 .
$$

The extensions $\dot{\Phi}^{ \pm}$of $\varphi$ satisfy such equations everywhere in the space domain, see (7). Considering that the traces $\dot{\Phi}_{j}^{ \pm}:=\partial_{t}^{j} \dot{\Phi}_{\mid t=0}^{ \pm}$are determined by (59) and that the equality $\left(\dot{\Phi}_{j}^{+}-\right.$ $\left.\dot{\Phi}_{j}^{-}\right)_{\mid x_{2}=0}=0$ defines their common value $\varphi_{j}:=\partial_{t}^{j} \varphi_{\mid t=0} \in H^{k+1-j}(\mathbb{R}), j=0, \ldots, k$, it follows that the compatibility conditions (62) and the relations (59) yield:

$$
\forall j=1, \ldots, k, \quad \varphi_{j}+\sum_{\ell=0}^{j-1} C_{j-1}^{\ell}\left(v_{\ell}^{ \pm}\right)_{\left.\right|_{x_{2}=0}} \partial_{x_{1}} \varphi_{j-1-\ell}-\left(u_{j-1}^{ \pm}\right)_{\left.\right|_{x_{2}=0}}=0,
$$

which is nothing but the expected compatibility conditions:

$$
\forall j=0, \ldots, k-1, \quad \partial_{t}^{j}\left(\partial_{t} \varphi+v_{\left.\right|_{x_{2}=0}}^{ \pm} \partial_{x_{1}} \varphi-u_{\left.\right|_{x_{2}=0}}^{ \pm}\right)_{\left.\right|_{t=0}}=0 .
$$

REMARK 6. - The restriction (63) that we impose on the initial data is specific to our problem. It does not follow automatically from a trace lemma. However, we shall see in the next paragraph that this restriction enables us to use a trace lemma for Sobolev spaces in a quarter space, which is more restrictive than a classical trace lemma in a half-space. However, this restriction is not essential and could be removed either to the price of some additional regularity on the initial data, or to the price of an additional step in our iteration scheme below.

There is a slight ambiguity in definition 1 . Indeed, the functions $\dot{\Phi}_{0}^{ \pm}$are not uniquely defined by $\varphi_{0}$. More rigorously, one could say that the initial data $\left(\dot{U}_{0}^{ \pm}, \varphi_{0}\right)$ are compatible if there exist some functions $\dot{\Phi}_{0}^{ \pm}$such that (54), (55), (62) and (63) hold. However, if one fixes once and for all a lifting operator and a $\mathcal{C}_{0}^{\infty}$ function in order to force (55), then there is no 
more ambiguity. We shall thus assume that such operators and functions have been fixed once and for all.

\subsection{Construction of an approximate solution}

Let us consider the nonlinear equations (3), (4) written in the compact form (8), (10). With a slight abuse of notation, we shall write $\mathbb{L}(U, \Phi)$ for the pair $\mathbb{L}\left(U^{ \pm}, \Phi^{ \pm}\right)$. We now introduce the following "approximate" solutions. These are solutions of (3), (4), (5), (6), (7) in the sense of Taylor's series at $t=0$. When not explicitly written, it is understood that functions $(U, \Phi)$ defined in the interior domain have + and - states.

In all what follows, $\varepsilon(\cdot)$ denotes a function that tends to 0 when its argument tends to 0 . For instance, the estimate (54), together with Sobolev's imbedding Theorem enables us to rewrite (61) as:

$$
\sum_{j=1}^{k}\left\|\dot{U}_{j}^{ \pm}\right\|_{H^{k+1 / 2-j}\left(\mathbb{R}_{+}^{2}\right)}+\sum_{\ell=1}^{k+1}\left\|\dot{\Phi}_{\ell}^{ \pm}\right\|_{H^{k+3 / 2-\ell}\left(\mathbb{R}_{+}^{2}\right)} \leq \varepsilon\left(\left\|\dot{U}_{0}^{ \pm}\right\|_{H^{k+1 / 2}\left(\mathbb{R}_{+}^{2}\right)}+\left\|\varphi_{0}\right\|_{H^{k+1}(\mathbb{R})}\right) .
$$

We consider the integer $\mu$ of Theorem 1 , some compatible initial data $\left(\dot{U}_{0}^{ \pm}, \varphi_{0}\right)$ in the sense of Definition 1, and we use the results of the previous paragraph with $k=\mu+7$. In particular, the initial data are compatible up to order $\mu+7$. We have the following result:

Lemma 3. - If $\dot{U}_{0}$ and $\varphi_{0}$ are sufficiently small, then there exist some functions $U^{a}, \Phi^{a}, \varphi^{a}$, such that $U^{a}-\bar{U}=\dot{U}^{a} \in H^{\mu+8}(\Omega), \Phi^{a \pm} \mp x_{2}=\dot{\Phi}^{a \pm} \in H^{\mu+9}(\Omega), \varphi^{a} \in H^{\mu+17 / 2}(\omega)$ and such that:

$$
\begin{aligned}
& \partial_{t}^{j} \mathbb{L}\left(U^{a}, \Phi^{a}\right)_{\mid t=0}=0, \quad \text { for } j=0, \ldots, \mu+6, \\
& \partial_{t} \Phi^{a}+v^{a} \partial_{x_{1}} \Phi^{a}-u^{a}=0 \\
& \varphi^{a}=\Phi_{\mid x_{2}=0}^{a+}=\Phi_{\mid x_{2}=0}^{a-}, \\
& \mathbb{B}\left(U_{\mid x_{2}=0}^{a}, \varphi^{a}\right)=0 .
\end{aligned}
$$

Moreover, one has the estimates:

$$
\begin{array}{r}
\left\|\dot{U}^{a}\right\|_{H^{\mu+8}(\Omega)}+\left\|\dot{\Phi}^{a}\right\|_{H^{\mu+9}(\Omega)}+\left\|\varphi^{a}\right\|_{H^{\mu+17 / 2}(\omega)} \\
\leq \varepsilon\left(\left\|\dot{U}_{0}^{ \pm}\right\|_{H^{\mu+15 / 2}\left(\mathbb{R}_{+}^{2}\right)}+\left\|\varphi_{0}\right\|_{H^{\mu+8}(\mathbb{R})}\right), \\
\forall(t, x) \in \Omega, \quad \partial_{x_{2}} \Phi^{a+}(t, x) \geq \frac{3}{4}, \quad \partial_{x_{2}} \Phi^{a-}(t, x) \leq-\frac{3}{4},
\end{array}
$$

and the following compact supports:

$$
\begin{aligned}
\operatorname{Supp}\left(\dot{U}^{a}, \dot{\Phi}^{a}\right) & \subset\left\{t \in[-T, T], x_{2} \geq 0, \sqrt{x_{1}^{2}+x_{2}^{2}} \leq 3\right\}, \\
\operatorname{Supp} \varphi^{a} & \subset\left\{t \in[-T, T],\left|x_{1}\right| \leq 3\right\} .
\end{aligned}
$$

The restrictions (63) of Definition 1 are important in order to ensure the RankineHugoniot conditions (67), (68). We shall see why in the proof. 
Proof. - Consider functions $\dot{\rho}^{a}, \dot{v}^{a} \in H^{\mu+8}(\Omega)$, and $\dot{\Phi}^{a} \in H^{\mu+9}(\Omega)$ such that:

$$
\begin{aligned}
\partial_{t}^{j}\left(\dot{\rho}^{a}, \dot{v}^{a}\right)_{\mid t=0} & =\left(\dot{\rho}_{j}, \dot{v}_{j}\right) \in H^{\mu+15 / 2-j}\left(\mathbb{R}_{+}^{2}\right), & & \text { for } j=0, \ldots, \mu+7, \\
\partial_{t}^{\ell}\left(\dot{\Phi}^{a}\right)_{\mid t=0} & =\dot{\Phi}_{\ell} \in H^{\mu+17 / 2-\ell}\left(\mathbb{R}_{+}^{2}\right), & & \text { for } \ell=0, \ldots, \mu+8,
\end{aligned}
$$

where the $\dot{\rho}_{j}, \dot{v}_{j}$ 's and the $\dot{\Phi}_{\ell}$ 's are given by Lemma 2 . Thanks to the compatibility conditions (62), and (63), one can even assume that $\dot{\rho}^{a}$ and $\dot{\Phi}^{a}$ satisfy the additional condition:

$$
\dot{\rho}_{\left.\right|_{x_{2}=0}}^{a+}=\dot{\rho}_{\left.\right|_{x_{2}=0}}^{a-}, \quad \dot{\Phi}_{\left.\right|_{x_{2}=0}}^{a+}=\dot{\Phi}_{\left.\right|_{x_{2}=0} ^{a-}}^{a-} .
$$

Indeed, it is enough to choose $\dot{\rho}^{a-}$ in an arbitrary way, then to choose $\dot{\rho}^{a+}-\dot{\rho}^{a-}$ such that:

$$
\begin{aligned}
\partial_{t}^{j}\left(\dot{\rho}^{a+}-\dot{\rho}^{a-}\right)_{\mid t=0} & =\left(\dot{\rho}_{j}^{+}-\dot{\rho}_{j}^{-}\right) \in H^{\mu+15 / 2-j}\left(\mathbb{R}_{+}^{2}\right), & & \text { for } j=0, \ldots, \mu+7, \\
\partial_{x_{2}}^{j}\left(\dot{\rho}^{a+}-\dot{\rho}^{a-}\right)_{\left.\right|_{x_{2}}=0} & =0, & & \text { for } j=0, \ldots, \mu+7 .
\end{aligned}
$$

Such a lifting is possible thanks to (63), see [22]. The argument for $\Phi^{a \pm}$ is the same.

Because the $\dot{U}_{j}$ 's, and the $\dot{\Phi}_{\ell}$ 's have a compact support, see Lemma 2, one can choose $\dot{\rho}^{a}$, $\dot{v}^{a}$ and $\dot{\Phi}^{a}$ that satisfy (71)up to multiplying by a $\mathcal{C}_{0}^{\infty}$ function. We define:

$$
\varphi^{a}:=\dot{\Phi}_{\left.\right|_{x_{2}=0}}^{a+}=\dot{\Phi}_{\left.\right|_{x_{2}=0} ^{a-}}^{a} \in H^{\mu+17 / 2}\left(\mathbb{R}^{2}\right), \quad \dot{u}^{a}:=\partial_{t} \dot{\Phi}^{a}+v^{a} \partial_{x_{1}} \dot{\Phi}^{a} \in H^{\mu+8}(\Omega),
$$

and we immediately note that $\dot{u}^{a}$, and $\varphi^{a}$ also satisfy (71). With this definition of $\dot{u}^{a}$ and $\varphi^{a}$, and our choice of $\dot{\rho}^{a}$, the relations (66), (68) are obvious. Moreover, the relations (59) show that $\dot{u}^{a}$ satisfies:

$$
\partial_{t}^{j}\left(\dot{u}^{a}\right)_{\mid t=0}=\dot{u}_{j} \in H^{\mu+15 / 2-j}\left(\mathbb{R}_{+}^{2}\right), \quad \text { for } j=0, \ldots, \mu+7 .
$$

The equations (58), (59) and (60) yield (65). The estimate (69) follows from (61), and from the continuity of the lifting operators. Eventually, (70) is a direct consequence of (69) and the Sobolev's imbedding Theorem, provided that the initial data are sufficiently small.

REMARK 7. - In the proof of Lemma 3, it is crucial to construct $\dot{u}^{a}$ in terms of $\dot{\Phi}^{a}$, and not the other way. Indeed, if one first defines $\dot{u}^{a}$ by lifting the traces $\dot{u}_{j}$ 's, and then defines $\dot{\Phi}^{a}$ as the solution to the transport equation:

$$
\partial_{t} \dot{\Phi}^{a}+v^{a} \partial_{x_{1}} \dot{\Phi}^{a}-\dot{u}^{a}=0
$$

there is no reason why $\dot{\Phi}^{a}$ should have a compact support in $(t, x)$ (we solve the transport equation for all times), and there is also no reason why $\dot{\Phi}^{a}$ should belong to $H^{\mu+9}(\Omega)$, because the coefficients $v^{a}, \dot{u}^{a}$ only belong to $H^{\mu+8}(\Omega)$.

The approximate solution $\left(U^{a}, \Phi^{a}\right)$ enables us to reformulate the original problem in a nonlinear problem with zero initial data. Introduce:

$$
\begin{cases}f^{a}:=-\mathbb{L}\left(U^{a}, \Phi^{a}\right), & t>0 \\ f^{a}:=0, & t<0 .\end{cases}
$$

Because $\dot{U}^{a}, \nabla \dot{\Phi}^{a} \in H^{\mu+8}(\Omega)$, (65) yields $f^{a} \in H^{\mu+7}(\Omega)$, and using (71) we get:

$$
\text { Supp } f^{a} \subset\left\{t \in[0, T], x_{2} \geq 0, \sqrt{x_{1}^{2}+x_{2}^{2}} \leq 3\right\} .
$$

From (69), we also get the estimate:

$$
\left\|f^{a}\right\|_{H^{\mu+7}(\Omega)} \leq \varepsilon\left(\left\|\dot{U}_{0}^{ \pm}\right\|_{H^{\mu+15 / 2\left(\mathbb{R}_{+}^{2}\right)}}+\left\|\varphi_{0}\right\|_{H^{\mu+8}(\mathbb{R})}\right) .
$$


For all real number $T>0$, we let $\Omega_{T}^{+}$, and $\omega_{T}^{+}$denote:

$$
\left.\omega_{T}^{+}:=\right] 0, T\left[\times \mathbb{R}, \quad \Omega_{T}^{+}:=\right] 0, T[\times \mathbb{R} \times] 0,+\infty\left[=\omega_{T}^{+} \times \mathbb{R}^{+} .\right.
$$

Given the approximate solution $\left(U^{a}, \Phi^{a}\right)$ of Lemma 3 , and $f^{a}$ defined by $(72),(U, \Phi)=$ $\left(U^{a}, \Phi^{a}\right)+(V, \Psi)$ is a solution on $\Omega_{T}^{+}$of (3), (4), (5), (6), (7), if $V=\left(V^{+}, V^{-}\right), \Psi=\left(\Psi^{+}, \Psi^{-}\right)$ satisfy the following system:

$$
\begin{array}{ll}
\mathcal{L}(V, \Psi)=f^{a}, & \text { in } \Omega_{T}, \\
\mathcal{E}(V, \Psi):=\partial_{t} \Psi+\left(v^{a}+v\right) \partial_{x_{1}} \Psi-u+v \partial_{x_{1}} \Phi^{a}=0, & \text { in } \Omega_{T}, \\
\Psi_{\left.\right|_{x_{2}=0}}^{+}=\Psi_{\left.\right|_{x_{2}}=0}^{-}=: \psi, & \text { on } \omega_{T}, \\
\mathcal{B}\left(V_{\left.\right|_{x_{2}}=0}, \psi\right)=0, & \text { on } \omega_{T}, \\
(V, \Psi)=0, & \text { for } t<0,
\end{array}
$$

where:

$$
\begin{aligned}
\mathcal{L}(V, \Psi) & :=\mathbb{L}\left(U^{a}+V, \Phi^{a}+\Psi\right)-\mathbb{L}\left(U^{a}, \Phi^{a}\right), \\
\mathcal{B}\left(V_{\left.\right|_{x_{2}}=0}, \psi\right) & :=\mathbb{B}\left(U_{\left.\right|_{x_{2}}=0}^{a}+V_{\left.\right|_{x_{2}}=0}, \varphi^{a}+\psi\right) .
\end{aligned}
$$

We note that the properties of the approximate solution imply that $(V, \Psi)=0$ satisfy (75) for $t<0$. Therefore the initial nonlinear problem on $\Omega_{T}^{+}$is now substituted for a problem on $\Omega_{T}$. The initial data (5) are absorbed into the source terms, and the problem has to be solved in the class of functions vanishing in the past, which is exactly the class of functions in which we have a well-posedness result for the linearized problem. 
Thanks to (69) and to the Sobolev imbedding Theorem, we see that for sufficiently small initial data, we have:

$$
\left\|\dot{U}^{a}\right\|_{W^{2, \infty}(\Omega)}+\left\|\dot{\Phi}^{a}\right\|_{W^{3, \infty}(\Omega)} \leq K_{0} / 2,
$$

where $K_{0}$ is given by Theorem 4 .

\section{Description of the iterative scheme}

We solve problem (75) by a Nash-Moser type iteration. (We refer to [2, 17, 19] for a general description of the method.) This method requires a family of smoothing operators whose construction is inspired from [14] and is detailed below. Also in this section, even if not explicitly written, it is understood that functions defined in the interior domain have a + and a - state.

\subsection{The smoothing operators}

We begin with a few notations. For $T>0, s \geq 0$, and $\gamma \geq 1$, we let:

$$
\mathcal{F}_{\gamma}^{s}\left(\Omega_{T}\right):=\left\{u \in H_{\gamma}^{s}\left(\Omega_{T}\right), \quad u=0 \text { for } t<0\right\} .
$$

This is a closed subspace of $H_{\gamma}^{s}\left(\Omega_{T}\right)$, that we equip with the induced norm. The definition of $\mathcal{F}_{\gamma}^{s}\left(\omega_{T}\right)$ is entirely similar. The following result follows from the method used in [1, section 4]:

Lemma 4. - There exists a family $\left\{S_{\theta}^{b}\right\}_{\theta \geq 1}$ of operators $S_{\theta}^{b}: \mathcal{F}_{\gamma}^{0}\left(\Omega_{T}\right) \longrightarrow \bigcap_{\beta \geq 0} \mathcal{F}_{\gamma}^{\beta}\left(\Omega_{T}\right)$, such that:

$$
\begin{aligned}
\left\|S_{\theta}^{b} u\right\|_{H_{\gamma}^{\beta}\left(\Omega_{T}\right)} & \leq C \theta^{(\beta-\alpha)_{+}}\|u\|_{H_{\gamma}^{\alpha}\left(\Omega_{T}\right)} & & \forall \alpha, \beta \geq 0, \\
\left\|S_{\theta}^{b} u-u\right\|_{H_{\gamma}^{\beta}\left(\Omega_{T}\right)} & \leq C \theta^{\beta-\alpha}\|u\|_{H_{\gamma}^{\alpha}\left(\Omega_{T}\right)} & & 0 \leq \beta \leq \alpha, \\
\left\|\frac{d}{d \theta} S_{\theta}^{b} u\right\|_{H_{\gamma}^{\beta}\left(\Omega_{T}\right)} & \leq C \theta^{\beta-\alpha-1}\|u\|_{H_{\gamma}^{\alpha}\left(\Omega_{T}\right)} & & \forall \alpha, \beta \geq 0 .
\end{aligned}
$$

Here, and in all what follows, we use the classical notation $(\beta-\alpha)_{+}:=\max (0, \beta-\alpha)$. The constants in the inequalities are uniform with respect to $\alpha, \beta$ when $\alpha, \beta$ belong to some bounded interval.

As noted in [14], the problem with the operators $S_{\theta}^{b}$ is that they do not respect the traces. More precisely, if $u$ and $v$ have the same trace on $\omega_{T}$ (when this trace is well-defined), there is no reason why $S_{\theta}^{b} u$ and $S_{\theta}^{b} v$ should have the same trace on $\omega_{T}$. To correct this defect, we introduce a lifting operator from the boundary to the interior:

Lemma 5. - Let $T>0, \gamma \geq 1$, and let $M \in \mathbb{N}, M \geq 1$. There exists an operator $\mathcal{R}_{T}$ such that:

- for all $s \in[1, M], \mathcal{R}_{T}$ is continuous from $\mathcal{F}_{\gamma}^{s}\left(\omega_{T}\right)$ to $\mathcal{F}_{\gamma}^{s+1 / 2}\left(\Omega_{T}\right)$,

- if $s \geq 1$, and $u \in \mathcal{F}_{\gamma}^{s}\left(\omega_{T}\right)$, then $\left(\mathcal{R}_{T} u\right)_{\left.\right|_{x_{2}=0}}=u$.

Extending first the functions of $\mathcal{F}_{\gamma}^{s}\left(\omega_{T}\right)$ to functions of $\mathcal{F}_{\gamma}^{s}(\omega)$, we are reduced to defining a lifting operator $\mathcal{R}$ from $\omega$ to $\Omega$. In this case, one can follow the construction of [14, chapter 5]. With the lifting operator $\mathcal{R}_{T}$, we prove the following (note that we now restrict Sobolev spaces to integer indices):

$4^{\mathrm{e}}$ SÉRIE - TOME $41-2008-\mathrm{N}^{\mathrm{o}} 1$ 
Proposition 4. - Let $T>0, \gamma \geq 1$, and let $M \in \mathbb{N}$, with $M \geq 4$. There exists a family $\left\{S_{\theta}\right\}_{\theta \geq 1}$ of operators

$$
S_{\theta}: \quad \mathcal{F}_{\gamma}^{3}\left(\Omega_{T}\right) \times \mathcal{F}_{\gamma}^{3}\left(\Omega_{T}\right) \longrightarrow \bigcap_{\beta \geq 3} \mathcal{F}_{\gamma}^{\beta}\left(\Omega_{T}\right) \times \mathcal{F}_{\gamma}^{\beta}\left(\Omega_{T}\right)
$$

and a constant $C>0$ (depending on $M$ ), such that:

$$
\begin{aligned}
\left\|S_{\theta} U\right\|_{H_{\gamma}^{\beta}\left(\Omega_{T}\right)} & \leq C \theta^{(\beta-\alpha)_{+}}\|U\|_{H_{\gamma}^{\alpha}\left(\Omega_{T}\right)}, & & \forall \alpha, \beta \in\{1, \ldots, M\}, \\
\left\|S_{\theta} U-U\right\|_{H_{\gamma}^{\beta}\left(\Omega_{T}\right)} & \leq C \theta^{\beta-\alpha}\|U\|_{H_{\gamma}^{\alpha}\left(\Omega_{T}\right)}, & & 1 \leq \beta \leq \alpha \leq M, \\
\left\|\frac{d}{d \theta} S_{\theta} U\right\|_{H_{\gamma}^{\beta}\left(\Omega_{T}\right)} & \leq C \theta^{\beta-\alpha-1}\|U\|_{H_{\gamma}^{\alpha}\left(\Omega_{T}\right)}, & & \forall \alpha, \beta \in\{1, \ldots, M\} .
\end{aligned}
$$

Moreover, (i) if $U=\left(u^{+}, u^{-}\right)$satisfies $u^{+}=u^{-}$on $\omega_{T}$, then $S_{\theta} u^{+}=S_{\theta} u^{-}$on $\omega_{T}$, (ii) the following estimate holds:

$$
\begin{aligned}
&\left\|\left(S_{\theta} u^{+}-S_{\theta} u^{-}\right)_{\left.\right|_{x_{2}=0}}\right\|_{H_{\gamma}^{\beta}\left(\omega_{T}\right)} \leq C \theta^{(\beta+1-\alpha)_{+}}\left\|\left(u^{+}-u^{-}\right)_{\left.\right|_{x_{2}=0}}\right\|_{H_{\gamma}^{\alpha}\left(\omega_{T}\right)}, \\
& \forall \alpha, \beta \in\{1, \ldots, M\} .
\end{aligned}
$$

There is another family of operators, still denoted $S_{\theta}$, that acts on functions that are defined on the boundary $\omega_{T}$, and that enjoy the properties (78), with the norms $\|\cdot\|_{H_{\gamma}^{\alpha}\left(\omega_{T}\right)}$.

Proof. - For $U=\left(u^{+}, u^{-}\right)$, we first define the "projection":

$$
\pi U:=\left(u^{+}-\frac{1}{2} \mathcal{R}_{T}\left(u^{+}-u^{-}\right)_{\left.\right|_{x_{2}=0}}, u^{-}+\frac{1}{2} \mathcal{R}_{T}\left(u^{+}-u^{-}\right)_{\left.\right|_{x_{2}=0}}\right),
$$

so that $\pi$ projects the pair $\left(u^{+}, u^{-}\right)$onto the set of pairs that have no jump on the boundary.

If $U=\left(u^{+}, u^{-}\right)$has no jump on the boundary, that is if $\pi U=U$, then we set $S_{\theta}^{\sharp} U:=$ $\pi S_{\theta}^{b} U$, where $S_{\theta}^{b}$ is given in Lemma 4. It is shown in [14] that the family $S_{\theta}^{\sharp}$ enjoys the properties (78). The family $S_{\theta}$ is now defined by $S_{\theta} U:=S_{\theta}^{\sharp}(\pi U)+S_{\theta}^{b}(U-\pi U)$. One easily checks that $S_{\theta}$ satisfies all the required properties.

In Proposition 4, the smoothing operators are defined on pairs of functions. However, we shall use the same notation $S_{\theta}$ for smoothing operators that act on vector valued functions of the type $\left(\rho^{ \pm}, v^{ \pm}, u^{ \pm}\right)$. Furthermore, one can follow a similar construction, and define a family of smoothing operators on $\mathcal{F}_{\gamma}^{3}\left(\Omega_{T}\right)$, still denoted $S_{\theta}$, that satisfies (78), and such that if the trace of $u$ on $\omega_{T}$ is zero, then the trace of $S_{\theta} u$ on $\omega_{T}$ is zero. The construction is similar to the construction of Proposition 4, except that here we deal with a single scalar function.

In Proposition 4, the value of the integer $M$ is not specified. At the end of section 7 the choice $M:=\mu+9$ will appear convenient.

\subsection{Description of the iterative scheme}

Let us describe the iterative scheme. The scheme starts from $V_{0}=0, \Psi_{0}=0, \psi_{0}=0$. Assume that $V_{k}, \Psi_{k}, \psi_{k}$ are already given for $k=0, \ldots, n$ and verify

$$
\begin{cases}\left(V_{k}, \Psi_{k}, \psi_{k}\right)=0, & \text { for } t<0, \\ \left(\Psi_{k}^{+}\right)_{\left.\right|_{x_{2}}=0}=\left(\Psi_{k}^{-}\right)_{\left.\right|_{x_{2}=0}}=\psi_{k}, & \text { on } \omega_{T} .\end{cases}
$$


As in [2], we consider

$$
V_{n+1}=V_{n}+\delta V_{n}, \quad \Psi_{n+1}=\Psi_{n}+\delta \Psi_{n}, \quad \psi_{n+1}=\psi_{n}+\delta \psi_{n},
$$

where the differences $\delta V_{n}, \delta \Psi_{n}, \delta \psi_{n}$ will be specified later on. Given $\theta_{0} \geq 1$, let us set $\theta_{n}:=$ $\left(\theta_{0}^{2}+n\right)^{1 / 2}$, and consider the smoothing operators $S_{\theta_{n}}$. We decompose

$$
\begin{aligned}
\mathcal{L}\left(V_{n+1}, \Psi_{n+1}\right)-\mathcal{L}\left(V_{n}, \Psi_{n}\right) & =\mathbb{L}\left(U^{a}+V_{n+1}, \Phi^{a}+\Psi_{n+1}\right)-\mathbb{L}\left(U^{a}+V_{n}, \Phi^{a}+\Psi_{n}\right) \\
& =\mathbb{L}^{\prime}\left(U^{a}+V_{n}, \Phi^{a}+\Psi_{n}\right)\left(\delta V_{n}, \delta \Psi_{n}\right)+e_{n}^{\prime} \\
& =\mathbb{L}^{\prime}\left(U^{a}+S_{\theta_{n}} V_{n}, \Phi^{a}+S_{\theta_{n}} \Psi_{n}\right)\left(\delta V_{n}, \delta \Psi_{n}\right)+e_{n}^{\prime}+e_{n}^{\prime \prime},
\end{aligned}
$$

where $e_{n}^{\prime}$ denotes the usual "quadratic" error of Newton's scheme, and $e_{n}^{\prime \prime}$ the "substitution" error. The operator $\mathbb{L}^{\prime}$ is given explicitly in (20).

Thanks to the properties of the smoothing operators, we have $\left(S_{\theta_{n}} \Psi_{n}^{+}\right)_{\left.\right|_{x_{2}=0}}=$ $\left(S_{\theta_{n}} \Psi_{n}^{-}\right)_{\left.\right|_{x_{2}=0}}$, see (79), and we denote $\psi_{n}^{\sharp}$ the common trace of these two functions. With this notation, we have

$$
\begin{aligned}
\mathcal{B}\left(\left(V_{n+1}\right)_{\left.\right|_{x_{2}=0}}, \psi_{n+1}\right)-\mathcal{B}\left(\left(V_{n}\right)_{\left.\right|_{x_{2}=0}}, \psi_{n}\right) & \\
& =\mathbb{B}^{\prime}\left(\left(U^{a}+V_{n}\right)_{\left.\right|_{x_{2}=0}}, \varphi^{a}+\psi_{n}\right)\left(\left(\delta V_{n}\right)_{\left.\right|_{x_{2}=0}}, \delta \psi_{n}\right)+\tilde{e}_{n}^{\prime} \\
& =\mathbb{B}^{\prime}\left(\left(U^{a}+S_{\theta_{n}} V_{n}\right)_{\left.\right|_{x_{2}=0}}, \varphi^{a}+\psi_{n}^{\sharp}\right)\left(\left(\delta V_{n}\right)_{\left.\right|_{x_{2}=0}}, \delta \psi_{n}\right)+\tilde{e}_{n}^{\prime}+\tilde{e}_{n}^{\prime \prime},
\end{aligned}
$$

where $\tilde{e}_{n}^{\prime}$ denotes the "quadratic" error, and $\tilde{e}_{n}^{\prime \prime}$ the "substitution" error. At this point a standard Nash-Moser iteration would require, as a main step, the resolution of a linearized problem of the form

$$
\left\{\begin{array}{l}
\mathbb{L}^{\prime}\left(U^{a}+S_{\theta_{n}} V_{n}, \Phi^{a}+S_{\theta_{n}} \Psi_{n}\right)\left(\delta V_{n}, \delta \Psi_{n}\right)=\ldots, \\
\mathbb{B}^{\prime}\left(\left(U^{a}+S_{\theta_{n}} V_{n}\right)_{\left.\right|_{x_{2}=0}}, \varphi^{a}+\psi_{n}^{\sharp}\right)\left(\left(\delta V_{n}\right)_{\left.\right|_{x_{2}=0}}, \delta \psi_{n}\right)=\ldots,
\end{array}\right.
$$

with smoothed coefficients, and suitable source terms on the right-hand side. However this is not possible in our case since inversion of the operator $\left(\mathbb{L}^{\prime}, \mathbb{B}^{\prime}\right)$, or more precisely of the effective linearized operator $\left(\mathbb{L}_{e}^{\prime}, \mathbb{B}_{e}^{\prime}\right)$, requires the linearization around a state satisfying the constraints (16), (17) and (18). We thus need to introduce a smooth modified state, denoted $V_{n+1 / 2}, \Psi_{n+1 / 2}, \psi_{n+1 / 2}$, that satisfies the above mentionned constraints. (The exact definition of this intermediate state is detailed in section 7.) Accordingly, we introduce the decompositions

$$
\begin{aligned}
& \mathcal{L}\left(V_{n+1}, \Psi_{n+1}\right)-\mathcal{L}\left(V_{n}, \Psi_{n}\right) \\
& \quad=\mathbb{L}^{\prime}\left(U^{a}+V_{n+1 / 2}, \Phi^{a}+\Psi_{n+1 / 2}\right)\left(\delta V_{n}, \delta \Psi_{n}\right)+e_{n}^{\prime}+e_{n}^{\prime \prime}+e_{n}^{\prime \prime \prime}, \\
& \mathcal{B}\left(\left(V_{n+1}\right)_{\left.\right|_{x_{2}=0}}, \psi_{n+1}\right)-\mathcal{B}\left(\left(V_{n}\right)_{\left.\right|_{x_{2}=0}}, \psi_{n}\right) \\
& \quad=\mathbb{B}^{\prime}\left(\left(U^{a}+V_{n+1 / 2}\right)_{\left.\right|_{x_{2}=0}}, \varphi^{a}+\psi_{n+1 / 2}\right)\left(\left(\delta V_{n}\right)_{\left.\right|_{x_{2}=0}}, \delta \psi_{n}\right)+\tilde{e}_{n}^{\prime}+\tilde{e}_{n}^{\prime \prime}+\tilde{e}_{n}^{\prime \prime \prime},
\end{aligned}
$$

where $e_{n}^{\prime \prime \prime}, \tilde{e}_{n}^{\prime \prime \prime}$ denote the second "substitution" errors.

The final step is the introduction of the "good unknown" (compare with (19)):

$$
\delta \dot{V}_{n}:=\delta V_{n}-\delta \Psi_{n} \frac{\partial_{x_{2}}\left(U^{a}+V_{n+1 / 2}\right)}{\partial_{x_{2}}\left(\Phi^{a}+\Psi_{n+1 / 2}\right)} .
$$


For the interior equations this leads to

$$
\begin{aligned}
& \mathcal{L}\left(V_{n+1}, \Psi_{n+1}\right)-\mathcal{L}\left(V_{n}, \Psi_{n}\right)=\mathbb{L}_{e}^{\prime}\left(U^{a}+V_{n+1 / 2}, \Phi^{a}+\Psi_{n+1 / 2}\right) \delta \dot{V}_{n} \\
& \quad+e_{n}^{\prime}+e_{n}^{\prime \prime}+e_{n}^{\prime \prime \prime}+\frac{\delta \Psi_{n}}{\partial_{x_{2}}\left(\Phi^{a}+\Psi_{n+1 / 2}\right)} \partial_{x_{2}}\left\{\mathbb{L}\left(U^{a}+V_{n+1 / 2}, \Phi^{a}+\Psi_{n+1 / 2}\right)\right\}
\end{aligned}
$$

recalling (20) and (22). For the boundary terms, we obtain

$$
\begin{aligned}
& \mathcal{B}\left(\left(V_{n+1}\right)_{\left.\right|_{x_{2}=0}}, \psi_{n+1}\right)-\mathcal{B}\left(\left(V_{n}\right)_{\left.\right|_{x_{2}=0}}, \psi_{n}\right) \\
& \quad=\mathbb{B}_{e}^{\prime}\left(\left(U^{a}+V_{n+1 / 2}\right)_{\left.\right|_{x_{2}=0}}, \varphi^{a}+\psi_{n+1 / 2}\right)\left(\left(\delta \dot{V}_{n}\right)_{\left.\right|_{x_{2}=0}}, \delta \psi_{n}\right)+\tilde{e}_{n}^{\prime}+\tilde{e}_{n}^{\prime \prime}+\tilde{e}_{n}^{\prime \prime \prime},
\end{aligned}
$$

where $\mathbb{B}_{e}^{\prime}$ is defined in (25). For the sake of brevity we set

$$
\begin{aligned}
& D_{n+1 / 2}:=\frac{1}{\partial_{x_{2}}\left(\Phi^{a}+\Psi_{n+1 / 2}\right)} \partial_{x_{2}}\left\{\mathbb{L}\left(U^{a}+V_{n+1 / 2}, \Phi^{a}+\Psi_{n+1 / 2}\right)\right\}, \\
& \mathbb{B}_{n+1 / 2}^{\prime}:=\mathbb{B}_{e}^{\prime}\left(\left(U^{a}+V_{n+1 / 2}\right)_{\left.\right|_{x_{2}=0}}, \varphi^{a}+\psi_{n+1 / 2}\right) .
\end{aligned}
$$

Let us also set

$$
e_{n}:=e_{n}^{\prime}+e_{n}^{\prime \prime}+e_{n}^{\prime \prime \prime}+D_{n+1 / 2} \delta \Psi_{n}, \quad \tilde{e}_{n}:=\tilde{e}_{n}^{\prime}+\tilde{e}_{n}^{\prime \prime}+\tilde{e}_{n}^{\prime \prime \prime} .
$$

The iteration proceeds as follows. Given

$$
\begin{aligned}
& V_{0}:=0, \quad \Psi_{0}:=0, \quad \psi_{0}:=0, \quad f_{0}:=S_{\theta_{0}} f^{a}, \quad g_{0}:=0, \quad E_{0}:=0, \quad \tilde{E}_{0}:=0, \\
& V_{1}, \ldots, V_{n}, \quad \Psi_{1}, \ldots, \Psi_{n}, \quad \psi_{1}, \ldots, \psi_{n}, \\
& f_{1}, \ldots, f_{n-1}, \quad g_{1}, \ldots, g_{n-1}, \quad e_{0}, \ldots, e_{n-1}, \quad \tilde{e}_{0}, \ldots, \tilde{e}_{n-1},
\end{aligned}
$$

we first compute for $n \geq 1$

$$
E_{n}:=\sum_{k=0}^{n-1} e_{k}, \quad \tilde{E}_{n}:=\sum_{k=0}^{n-1} \tilde{e}_{k} .
$$

These are the accumulated errors at the step $n$. Then we compute $f_{n}$, and $g_{n}$ from the equations:

$$
\sum_{k=0}^{n} f_{k}+S_{\theta_{n}} E_{n}=S_{\theta_{n}} f^{a}, \quad \sum_{k=0}^{n} g_{k}+S_{\theta_{n}} \tilde{E}_{n}=0,
$$

and we solve the linear problem

$$
\begin{array}{ll}
\mathbb{L}_{e}^{\prime}\left(U^{a}+V_{n+1 / 2}, \Phi^{a}+\Psi_{n+1 / 2}\right) \delta \dot{V}_{n}=f_{n} & \text { in } \Omega_{T}, \\
\mathbb{B}_{n+1 / 2}^{\prime}\left(\left(\delta \dot{V}_{n}\right)_{\left.\right|_{x_{2}}=0}, \delta \psi_{n}\right)=g_{n} & \text { on } \omega_{T}, \\
\delta \dot{V}_{n}=0, \quad \delta \psi_{n}=0 & \text { for } t<0,
\end{array}
$$

finding $\left(\delta \dot{V}_{n}, \delta \psi_{n}\right)$. Now we need to construct $\delta \Psi_{n}=\left(\delta \Psi_{n}^{+}, \delta \Psi_{n}^{-}\right)$that satisfies $\left(\delta \Psi_{n}^{ \pm}\right)_{\left.\right|_{x_{2}=0}}=$ $\delta \psi_{n}$. Using the explicit expression of the boundary conditions in (87), see (25) and (24), we 
first note that $\delta \psi_{n}$ solves the equation:

$$
\begin{aligned}
& \partial_{t} \delta \psi_{n}+\left(v^{a+}+v_{n+1 / 2}^{+}\right)_{\left.\right|_{x_{2}=0}} \partial_{x_{1}} \delta \psi_{n} \\
& +\left\{\partial_{x_{1}}\left(\varphi^{a}+\psi_{n+1 / 2}\right) \frac{\left.\partial_{x_{2}}\left(v^{a+}+v_{n+1 / 2}^{+}\right)\right)_{\left.\right|_{x_{2}}=0}}{\partial_{x_{2}}\left(\Phi^{a+}+\Psi_{n+1 / 2}^{+}\right)_{\left.\right|_{x_{2}}=0}}-\frac{\partial_{x_{2}}\left(u^{a+}+u_{n+1 / 2}^{+}\right)_{\left.\right|_{x_{2}}=0}}{\partial_{x_{2}}\left(\Phi^{a+}+\Psi_{n+1 / 2}^{+}\right)_{\left.\right|_{x_{2}}=0}}\right\} \delta \psi_{n} \\
& +\partial_{x_{1}}\left(\varphi^{a}+\psi_{n+1 / 2}\right)\left(\delta \dot{v}_{n}^{+}\right)_{\left.\right|_{x_{2}=0}}-\left(\delta \dot{u}_{n}^{+}\right)_{\left.\right|_{x_{2}=0}=g_{n, 2}},
\end{aligned}
$$

and the equation

$$
\begin{aligned}
& \partial_{t} \delta \psi_{n}+\left(v^{a-}+v_{n+1 / 2}^{-}\right)_{\left.\right|_{x_{2}=0}} \partial_{x_{1}} \delta \psi_{n} \\
& +\left\{\partial_{x_{1}}\left(\varphi^{a}+\psi_{n+1 / 2}\right) \frac{\left.\partial_{x_{2}}\left(v^{a-}+v_{n+1 / 2}^{-}\right)\right)_{\left.\right|_{x_{2}}=0}}{\partial_{x_{2}}\left(\Phi^{a-}+\Psi_{n+1 / 2}^{-}\right)_{\left.\right|_{x_{2}}=0}}-\frac{\left.\partial_{x_{2}}\left(u^{a-}+u_{n+1 / 2}^{-}\right)\right|_{x_{2}=0}}{\partial_{x_{2}}\left(\Phi^{a-}+\Psi_{n+1 / 2}^{-}\right)_{\left.\right|_{x_{2}}=0}}\right\} \delta \psi_{n} \\
& +\partial_{x_{1}}\left(\varphi^{a}+\psi_{n+1 / 2}\right)\left(\delta \dot{v}_{n}^{-}\right)_{\left.\right|_{x_{2}=0}}-\left(\delta \dot{u}_{n}^{-}\right)_{\left.\right|_{x_{2}=0}}=g_{n, 2}-g_{n, 1} .
\end{aligned}
$$

We shall thus define $\delta \Psi_{n}^{+}, \delta \Psi_{n}^{-}$as the solutions to the following equations:

$$
\begin{aligned}
\text { (90) } & \partial_{t} \delta \Psi_{n}^{+}+\left(v^{a+}+v_{n+1 / 2}^{+}\right) \partial_{x_{1}} \delta \Psi_{n}^{+} \\
& +\left\{\partial_{x_{1}}\left(\Phi^{a+}+\Psi_{n+1 / 2}^{+}\right) \frac{\partial_{x_{2}}\left(v^{a+}+v_{n+1 / 2}^{+}\right)}{\partial_{x_{2}}\left(\Phi^{a+}+\Psi_{n+1 / 2}^{+}\right)}-\frac{\partial_{x_{2}}\left(u^{a+}+u_{n+1 / 2}^{+}\right)}{\partial_{x_{2}}\left(\Phi^{a+}+\Psi_{n+1 / 2}^{+}\right)}\right\} \delta \Psi_{n}^{+} \\
& +\partial_{x_{1}}\left(\Phi^{a+}+\Psi_{n+1 / 2}^{+}\right) \delta \dot{v}_{n}^{+}-\delta \dot{u}_{n}^{+}=\mathcal{R}_{T} g_{n, 2}+h_{n}^{+}, \\
\text {(91) } & \partial_{t} \delta \Psi_{n}^{-}+\left(v^{a-}+v_{n+1 / 2}^{-}\right) \partial_{x_{1}} \delta \Psi_{n}^{-} \\
& +\left\{\partial_{x_{1}}\left(\Phi^{a-}+\Psi_{n+1 / 2}^{-}\right) \frac{\partial_{x_{2}}\left(v^{a-}+v_{n+1 / 2}^{-}\right)}{\partial_{x_{2}}\left(\Phi^{a-}+\Psi_{n+1 / 2}^{-}\right)}-\frac{\partial_{x_{2}}\left(u^{a-}+u_{n+1 / 2}^{-}\right)}{\partial_{x_{2}}\left(\Phi^{a-}+\Psi_{n+1 / 2}^{-}\right)}\right\} \delta \Psi_{n}^{-} \\
& +\partial_{x_{1}}\left(\Phi^{a-}+\Psi_{n+1 / 2}^{-}\right) \delta \dot{v}_{n}^{-}-\delta \dot{u}_{n}^{-}=\mathcal{R}_{T}\left(g_{n, 2}-g_{n, 1}\right)+h_{n}^{-} .
\end{aligned}
$$

In order to compute the source terms $h_{n}^{ \pm}$, we use a decomposition for the operator $\mathcal{E}$ that is similar to (82). Recall that this operator is defined in (75). We have:

$$
\mathcal{E}\left(V_{n+1}, \Psi_{n+1}\right)-\mathcal{E}\left(V_{n}, \Psi_{n}\right)=\mathcal{E}^{\prime}\left(V_{n+1 / 2}, \Psi_{n+1 / 2}\right)\left(\delta V_{n}, \delta \Psi_{n}\right)+\hat{e}_{n}^{\prime}+\hat{e}_{n}^{\prime \prime}+\hat{e}_{n}^{\prime \prime \prime},
$$

where $\hat{e}_{n}^{\prime}$ is the "quadratic" error, $\hat{e}_{n}^{\prime \prime}$ is the first "substitution" error, and $\hat{e}_{n}^{\prime \prime \prime}$ is the second "substitution" error. We denote

$$
\hat{e}_{n}:=\hat{e}_{n}^{\prime}+\hat{e}_{n}^{\prime \prime}+\hat{e}_{n}^{\prime \prime \prime}, \quad \hat{E}_{n}:=\sum_{k=0}^{n-1} \hat{e}_{k} .
$$

Using the good unknown (81), and omitting the \pm superscripts, we compute

$$
\begin{aligned}
& \mathcal{E}^{\prime}\left(V_{n+1 / 2}, \Psi_{n+1 / 2}\right)\left(\delta V_{n}, \delta \Psi_{n}\right)=\partial_{t} \delta \Psi_{n}+\left(v^{a}+v_{n+1 / 2}\right) \partial_{x_{1}} \delta \Psi_{n} \\
& \quad+\left\{\partial_{x_{1}}\left(\Phi^{a}+\Psi_{n+1 / 2}\right) \frac{\partial_{x_{2}}\left(v^{a}+v_{n+1 / 2}\right)}{\partial_{x_{2}}\left(\Phi^{a}+\Psi_{n+1 / 2}\right)}-\frac{\partial_{x_{2}}\left(u^{a}+u_{n+1 / 2}\right)}{\partial_{x_{2}}\left(\Phi^{a}+\Psi_{n+1 / 2}\right)}\right\} \delta \Psi_{n} \\
& \quad+\partial_{x_{1}}\left(\Phi^{a}+\Psi_{n+1 / 2}\right) \delta \dot{v}_{n}-\delta \dot{u}_{n} .
\end{aligned}
$$

Consequently, (90) and (92) yield

$$
\mathcal{E}\left(V_{n+1}^{+}, \Psi_{n+1}^{+}\right)-\mathcal{E}\left(V_{n}^{+}, \Psi_{n}^{+}\right)=\mathcal{R}_{T} g_{n, 2}+h_{n}^{+}+\hat{e}_{n}^{+} .
$$


Summing these relations, and using $\mathcal{E}\left(V_{0}^{+}, \Psi_{0}^{+}\right)=0$, we get

$$
\begin{aligned}
\mathcal{E}\left(V_{n+1}^{+}, \Psi_{n+1}^{+}\right) & =\mathcal{R}_{T}\left(\sum_{k=0}^{n} g_{k, 2}\right)+\sum_{k=0}^{n} h_{k}^{+}+\hat{E}_{n+1}^{+} \\
& =\mathcal{R}_{T}\left(\mathcal{E}\left(\left(V_{n+1}^{+}\right)_{\left.\right|_{x_{2}=0}}, \psi_{n+1}\right)-\tilde{E}_{n+1,2}\right)+\sum_{k=0}^{n} h_{k}^{+}+\hat{E}_{n+1}^{+},
\end{aligned}
$$

where in the last equality we have summed (83) and used the relation

$$
\left(\mathcal{B}\left(\left(V_{n+1}\right)_{\left.\right|_{x_{2}=0}}, \psi_{n+1}\right)\right)_{2}=\mathcal{E}\left(\left(V_{n+1}^{+}\right)_{\left.\right|_{x_{2}=0}}, \psi_{n+1}\right),
$$

see (76) and (10). The previous relations lead to the following definition of the source term $h_{n}^{+}$:

$$
\sum_{k=0}^{n} h_{k}^{+}+S_{\theta_{n}}\left(\hat{E}_{n}^{+}-\mathcal{R}_{T} \tilde{E}_{n, 2}\right)=0 .
$$

The definition of $h_{n}^{-}$is similar:

$$
\sum_{k=0}^{n} h_{k}^{-}+S_{\theta_{n}}\left(\hat{E}_{n}^{-}-\mathcal{R}_{T} \tilde{E}_{n, 2}+\mathcal{R}_{T} \tilde{E}_{n, 1}\right)=0 .
$$

As in [14], one can check that the source terms $h_{k}^{ \pm}$vanish in the past, and that their trace on $\omega_{T}$ vanishes, so that the unique smooth solutions to (90) and (91) vanish in the past, and satisfy the continuity condition $\left(\delta \Psi_{n}^{ \pm}\right)_{\left.\right|_{x_{2}=0}}=\delta \psi_{n}$.

Once $\delta \Psi_{n}$ is computed, the function $\delta V_{n}$ is obtained from (81), and the functions $V_{n+1}$, $\Psi_{n+1}, \psi_{n+1}$ are obtained from (80). Finally, we compute $e_{n}, \hat{e}_{n}, \tilde{e}_{n}$ from

$$
\begin{aligned}
\mathcal{L}\left(V_{n+1}, \Psi_{n+1}\right)-\mathcal{L}\left(V_{n}, \Psi_{n}\right) & =f_{n}+e_{n}, \\
\mathcal{E}\left(V_{n+1}^{+}, \Psi_{n+1}^{+}\right)-\mathcal{E}\left(V_{n}^{+}, \Psi_{n}^{+}\right) & =\mathcal{R}_{T} g_{n, 2}+h_{n}^{+}+\hat{e}_{n}^{+}, \\
\mathcal{E}\left(V_{n+1}^{-}, \Psi_{n+1}^{-}\right)-\mathcal{E}\left(V_{n}^{-}, \Psi_{n}^{-}\right) & =\mathcal{R}_{T}\left(g_{n, 2}-g_{n, 1}\right)+h_{n}^{-}+\hat{e}_{n}^{-}, \\
\mathcal{B}\left(\left(V_{n+1}\right)_{\left.\right|_{x_{2}=0}}, \psi_{n+1}\right)-\mathcal{B}\left(\left(V_{n}\right)_{\left.\right|_{x_{2}}=0}, \psi_{n}\right) & =g_{n}+\tilde{e}_{n} .
\end{aligned}
$$

To compute $V_{1}, \Psi_{1}, \psi_{1}$ we only consider steps (87), (90), (91), (95) for $n=0$.

Adding (95) from 0 to $N$ and combining with (86) give

$$
\begin{aligned}
\mathcal{L}\left(V_{N+1}, \Psi_{N+1}\right)-f^{a}= & \left(S_{\theta_{N}}-I\right) f^{a}+\left(I-S_{\theta_{N}}\right) E_{N}+e_{N}, \\
\mathcal{E}\left(V_{N+1}^{+}, \Psi_{N+1}^{+}\right)= & \mathcal{R}_{T}\left(\mathcal{E}\left(\left(V_{N+1}^{+}\right)_{\left.\right|_{x_{2}=0}}, \psi_{N+1}\right)\right) \\
& +\left(I-S_{\theta_{N}}\right)\left(\hat{E}_{N}^{+}-\mathcal{R}_{T} \tilde{E}_{N, 2}\right)+\hat{e}_{N}^{+}-\mathcal{R}_{T} \tilde{e}_{N, 2}, \\
\mathcal{E}\left(V_{N+1}^{-}, \Psi_{N+1}^{-}\right)= & \mathcal{R}_{T}\left(\mathcal{E}\left(\left(V_{N+1}^{-}\right)_{\left.\right|_{x_{2}=0}}, \psi_{N+1}\right)\right) \\
& +\left(I-S_{\theta_{N}}\right)\left(\hat{E}_{N}^{-}-\mathcal{R}_{T}\left(\tilde{E}_{N, 2}-\tilde{E}_{N, 1}\right)\right)+\hat{e}_{N}^{-}-\mathcal{R}_{T}\left(\tilde{e}_{N, 2}-\tilde{e}_{N, 1}\right), \\
\mathcal{B}\left(\left(V_{N+1}\right)_{\left.\right|_{x_{2}=0}}, \psi_{N+1}\right)= & \left(I-S_{\theta_{N}}\right) \tilde{E}_{N}+\tilde{e}_{N} .
\end{aligned}
$$

Because $S_{\theta_{N}} \rightarrow I$ as $N \rightarrow+\infty$, and since we expect $\left(e_{N}, \hat{e}_{n}, \tilde{e}_{N}\right) \rightarrow 0$, we will formally obtain the solution of the problem $(75)$ from $\mathcal{L}\left(V_{N+1}, \Psi_{N+1}\right) \rightarrow f^{a}, \mathcal{B}\left(\left(V_{N+1}\right)_{\left.\right|_{x_{2}}=0}, \psi_{N+1}\right) \rightarrow 0$, and $\mathcal{E}\left(V_{N+1}, \Psi_{N+1}\right) \rightarrow 0$. 


\section{More tame estimates}

In this section, we are going to summarize the estimates that will be needed in the proof of Theorem 1. We recall that $\mu$ denotes the integer given in the assumptions of Theorem 1. Also in Propositions 5 and 6 below, we use an integer $\tilde{\alpha}$ that will be specified in section 7. (We shall see that a suitable choice for this integer is $\tilde{\alpha}=\mu+5$.)

\subsection{Tame estimate for the second derivatives}

For the control of the errors $e_{n}^{\prime}, \hat{e}_{n}^{\prime}, \tilde{e}_{n}^{\prime}$ etc. in the iteration scheme, we need to estimate the second derivative of the operators $\mathbb{L}, \mathcal{E}$, and $\mathbb{B}$. These operators are defined in (8), (10) and (75). We are going to derive these estimates with some weaker smoothness assumption for the coefficients, than what was needed in section 3. More precisely, we consider a fixed time $T>0$, and we assume that

$$
\dot{U}_{r, l}, \dot{\Phi}_{r, l} \in W^{1, \infty}\left(\Omega_{T}\right), \quad\left\|\left(\dot{U}_{r, l}, \dot{\Phi}_{r, l}\right)\right\|_{W^{1, \infty}\left(\Omega_{T}\right)} \leq \widetilde{K},
$$

where $\widetilde{K}$ is a positive constant. Let $\tilde{\alpha}$ be a sufficiently large integer that will be chosen later on. We have the following result:

Proposition 5. - Let $m \in \mathbb{N}, m \in[3, \tilde{\alpha}-1]$, and let $T>0$. Assume that the perturbations $\dot{U}_{r, l}, \dot{\Phi}_{r, l}$ satisfy (96), and

$$
\forall \gamma \geq 1, \quad\left(\dot{U}_{r, l}, \dot{\Phi}_{r, l}\right) \in H_{\gamma}^{\tilde{\alpha}}\left(\Omega_{T}\right) .
$$

Then there exist two constants $\widetilde{K_{0}}>0$, and $C>0$, that do not depend on $T$ and $\gamma$, such that, if $\widetilde{K} \leq \widetilde{K_{0}}, \gamma \geq 1$, and if $\left(V^{\prime}, \Psi^{\prime}\right),\left(V^{\prime \prime}, \Psi^{\prime \prime}\right) \in H_{\gamma}^{m+1}\left(\Omega_{T}\right)$, then one has

$$
\begin{aligned}
\| \mathbb{L}^{\prime \prime}\left(U_{r, l}, \Phi_{r, l}\right) & \left(\left(V^{\prime}, \Psi^{\prime}\right),\left(V^{\prime \prime}, \Psi^{\prime \prime}\right)\right) \|_{H_{\gamma}^{m}\left(\Omega_{T}\right)} \\
\leq & C\left\{\|(\dot{U}, \dot{\Phi})\|_{H_{\gamma}^{m+1}\left(\Omega_{T}\right)}\left\|\left(V^{\prime}, \Psi^{\prime}\right)\right\|_{W^{1, \infty}\left(\Omega_{T}\right)}\left\|\left(V^{\prime \prime}, \Psi^{\prime \prime}\right)\right\|_{W^{1, \infty}\left(\Omega_{T}\right)}\right. \\
& +\left\|\left(V^{\prime}, \Psi^{\prime}\right)\right\|_{H_{\gamma}^{m+1}\left(\Omega_{T}\right)}\left\|\left(V^{\prime \prime}, \Psi^{\prime \prime}\right)\right\|_{W^{1, \infty}\left(\Omega_{T}\right)} \\
& \left.+\left\|\left(V^{\prime \prime}, \Psi^{\prime \prime}\right)\right\|_{H_{\gamma}^{m+1}\left(\Omega_{T}\right)}\left\|\left(V^{\prime}, \Psi^{\prime}\right)\right\|_{W^{1, \infty}\left(\Omega_{T}\right)}\right\},
\end{aligned}
$$

and

$$
\begin{aligned}
& \left\|\mathcal{E}^{\prime \prime}\left(\left(V^{\prime}, \Psi^{\prime}\right),\left(V^{\prime \prime}, \Psi^{\prime \prime}\right)\right)\right\|_{H_{\gamma}^{m}\left(\Omega_{T}\right)} \leq C\left\{\left\|V^{\prime}\right\|_{H_{\gamma}^{m}\left(\Omega_{T}\right)}\left\|\Psi^{\prime \prime}\right\|_{W^{1, \infty}\left(\Omega_{T}\right)}\right. \\
& +\left\|V^{\prime}\right\|_{L^{\infty}\left(\Omega_{T}\right)}\left\|\Psi^{\prime \prime}\right\|_{H_{\gamma}^{m+1}\left(\Omega_{T}\right)}+\left\|V^{\prime \prime}\right\|_{H_{\gamma}^{m}\left(\Omega_{T}\right)}\left\|\Psi^{\prime}\right\|_{W^{1, \infty}\left(\Omega_{T}\right)} \\
& \left.+\left\|V^{\prime \prime}\right\|_{L^{\infty}\left(\Omega_{T}\right)}\left\|\Psi^{\prime}\right\|_{H_{\gamma}^{m+1}\left(\Omega_{T}\right)}\right\} .
\end{aligned}
$$

$$
\begin{aligned}
& \left\|\mathbb{B}^{\prime \prime}\left(\left(W^{\prime}, \psi^{\prime}\right),\left(W^{\prime \prime}, \psi^{\prime \prime}\right)\right)\right\|_{H_{\gamma}^{m}\left(\omega_{T}\right)} \leq C\left\{\left\|W^{\prime}\right\|_{H_{\gamma}^{m}\left(\omega_{T}\right)}\left\|\psi^{\prime \prime}\right\|_{W^{1, \infty}\left(\omega_{T}\right)}\right. \\
& \quad+\left\|W^{\prime}\right\|_{L^{\infty}\left(\omega_{T}\right)}\left\|\psi^{\prime \prime}\right\|_{H_{\gamma}^{m+1}\left(\omega_{T}\right)}+\left\|W^{\prime \prime}\right\|_{H_{\gamma}^{m}\left(\omega_{T}\right)}\left\|\psi^{\prime}\right\|_{W^{1, \infty}\left(\omega_{T}\right)} \\
& \left.\quad+\left\|W^{\prime \prime}\right\|_{L^{\infty}\left(\omega_{T}\right)}\left\|\psi^{\prime}\right\|_{H_{\gamma}^{m+1}\left(\omega_{T}\right)}\right\} .
\end{aligned}
$$


Proof. - The proof follows from the long, but straightforward calculation of the explicit expression of $\mathbb{L}^{\prime \prime}, \mathcal{E}^{\prime \prime}, \mathbb{B}^{\prime \prime}$, from Theorem 9 and Theorem 10 . The constant $\widetilde{K}_{0}$ is fixed so that under the constraint $\widetilde{K} \leq \widetilde{K}_{0}$, the map $U_{r, l}$ takes its values in a fixed compact domain of the hyperbolicity region, and $\left\|\nabla \dot{\Phi}_{r, l}\right\|_{W^{1, \infty}\left(\Omega_{T}\right)} \leq 1 / 2$.

The estimates (97), (98), (99) hold for every $m$, with a constant $C$ that may depend on $m$. Since in Proposition 5, $m$ is taken in a bounded interval, the constant $C$ may be assumed to be independent of $m$. The same remark holds for Proposition 6 in the next subsection.

\subsection{Tame estimate for the linearized problem}

We conclude this section by writing the tame estimate (52) in a more convenient form:

Proposition 6. - Let $T>0$, and $m \in \mathbb{N}, m \in[3, \tilde{\alpha}]$. Let us assume that the hypotheses of Theorem 4 hold and that the perturbations satisfy

$$
\|(\dot{U}, \nabla \dot{\Phi})\|_{H_{\gamma}^{5}\left(\Omega_{T}\right)}+\left\|\left(\dot{U}, \partial_{x_{2}} \dot{U}, \nabla \dot{\Phi}\right)_{\left.\right|_{x_{2}=0}}\right\|_{H_{\gamma}^{4}\left(\omega_{T}\right)} \leq K .
$$

Then there exist some constants $C>0$ and $\gamma \geq 1$, that only depend on $K_{0}$ (that is given by Theorem 4), such that, if $K \leq K_{0}$, and if $\left(f_{ \pm}, g\right) \in H^{m+1}\left(\Omega_{T}\right) \times H^{m+1}\left(\omega_{T}\right)$ vanish in the past, then there exists a unique solution $\left(\dot{V}_{ \pm}, \psi\right) \in H^{m}\left(\Omega_{T}\right) \times H^{m+1}\left(\omega_{T}\right)$ to (22), (25) that vanishes in the past. Moreover the following estimate holds:

$$
\begin{aligned}
\|\dot{V}\|_{H_{\gamma}^{m}\left(\Omega_{T}\right)}+\left\|\mathbb{P}(\varphi) \dot{V}_{\left.\right|_{x_{2}=0}}\right\|_{H_{\gamma}^{m}\left(\omega_{T}\right)}+ & \|\psi\|_{H_{\gamma}^{m+1}\left(\omega_{T}\right)} \leq C\left\{\|f\|_{H_{\gamma}^{m+1}\left(\Omega_{T}\right)}+\|g\|_{H_{\gamma}^{m+1}\left(\omega_{T}\right)}\right. \\
& \left.+\left(\|f\|_{H_{\gamma}^{4}\left(\Omega_{T}\right)}+\|g\|_{H_{\gamma}^{4}\left(\omega_{T}\right)}\right)\|(\dot{U}, \dot{\Phi})\|_{H_{\gamma}^{m+3}\left(\Omega_{T}\right)}\right\} .
\end{aligned}
$$

Proof. - Theorem 3 gives the well-posedness in $L^{2}$, and we omit the proof that for source terms $\left(f_{ \pm}, g\right) \in H^{m+1}\left(\Omega_{T}\right) \times H^{m+1}\left(\omega_{T}\right)$ vanishing in the past, the solution $(\dot{V}, \psi)$ belongs to $H^{m}\left(\Omega_{T}\right) \times H^{m+1}\left(\omega_{T}\right)$ and satisfies (52) for $\gamma \geq \gamma_{m}$. In order to show (101), we first fix $\gamma$ greater than the maximum of $\gamma_{0}, \ldots, \gamma_{\tilde{\alpha}}$. From (52) with $m=3$, Theorem 11 and the Sobolev imbedding Theorem, we get

$$
\|\dot{V}\|_{W^{1, \infty}\left(\Omega_{T}\right)}+\left\|\mathbb{P}(\varphi) \dot{V}_{\left.\right|_{x_{2}=0}}\right\|_{L^{\infty}\left(\omega_{T}\right)}+\|\psi\|_{W^{1, \infty}\left(\omega_{T}\right)} \leq C\left(\|f\|_{H_{\gamma}^{4}\left(\Omega_{T}\right)}+\|g\|_{H_{\gamma}^{4}\left(\omega_{T}\right)}\right),
$$

by choosing $K_{0}$ small enough (observe that this choice depends on $T$, and on $\gamma$ that has been fixed as above). Finally we use this inequality in (52).

Notice that from now on $\gamma$ is fixed, as detailed in the proof of Proposition 6.

\section{Proof of the main result}

We recall that the sequence $\left(\theta_{n}\right)$ is defined by $\theta_{0} \geq 1, \theta_{n}:=\left(\theta_{0}^{2}+n\right)^{1 / 2}$, and we denote $\Delta_{n}:=\theta_{n+1}-\theta_{n}$. In particular, the sequence $\left(\Delta_{n}\right)$ is decreasing, and tends to zero. Moreover, one has

$$
\forall n \in \mathbb{N}, \quad \frac{1}{3 \theta_{n}} \leq \Delta_{n}=\sqrt{\theta_{n}^{2}+1}-\theta_{n} \leq \frac{1}{2 \theta_{n}} .
$$




\subsection{Introduction of the iterative scheme}

Given a small number $\delta>0$, and an integer $\tilde{\alpha}$ that will be chosen later on, let us assume that the following estimate holds:

$$
\left\|\dot{U}^{a}\right\|_{H_{\gamma}^{\tilde{\alpha}+3}\left(\Omega_{T}\right)}+\left\|\dot{\Phi}^{a}\right\|_{H_{\gamma}^{\tilde{\alpha}+4}\left(\Omega_{T}\right)}+\left\|\varphi^{a}\right\|_{H_{\gamma}^{\tilde{\alpha}+7 / 2}\left(\omega_{T}\right)}+\left\|f^{a}\right\|_{H_{\gamma}^{\tilde{\alpha}+2}\left(\Omega_{T}\right)} \leq \delta .
$$

Given the integer $\alpha:=\mu+1$, our inductive assumption reads:

$$
\left(H_{n-1}\right)\left\{\begin{array}{c}
\text { a) } \forall k=0, \ldots, n-1, \quad \forall s \in[3, \tilde{\alpha}] \cap \mathbb{N}, \\
\left\|\left(\delta V_{k}, \delta \Psi_{k}\right)\right\|_{H_{\gamma}^{s}\left(\Omega_{T}\right)}+\left\|\delta \psi_{k}\right\|_{H_{\gamma}^{s+1}\left(\omega_{T}\right)} \leq \delta \theta_{k}^{s-\alpha-1} \Delta_{k}, \\
\text { b) } \forall k=0, \ldots, n-1, \quad \forall s \in[3, \tilde{\alpha}-2] \cap \mathbb{N}, \\
\quad\left\|\mathcal{L}\left(V_{k}, \Psi_{k}\right)-f^{a}\right\|_{H_{\gamma}^{s}\left(\Omega_{T}\right)} \leq 2 \delta \theta_{k}^{s-\alpha-1}, \\
\text { c) } \forall k=0, \ldots, n-1, \quad \forall s \in[4, \alpha] \cap \mathbb{N},\left\|\mathcal{B}\left(V_{k}, \psi_{k}\right)\right\|_{H_{\gamma}^{s}\left(\omega_{T}\right)} \leq \delta \theta_{k}^{s-\alpha-1}, \\
\text { d) } \forall k=0, \ldots, n-1,\left\|\mathcal{E}\left(V_{k}, \Psi_{k}\right)\right\|_{H_{\gamma}^{3}\left(\Omega_{T}\right)} \leq \delta \theta_{k}^{2-\alpha}
\end{array}\right.
$$

For $k=0, \ldots, n$, the functions $V_{k}, \Psi_{k}, \psi_{k}$ are also assumed to satisfy (79).

The first task is to prove that for a suitable choice of the parameters $\theta_{0} \geq 1$ and $\delta>0$, and for $f^{a}$ small enough, $\left(H_{n-1}\right)$ implies $\left(H_{n}\right)$. In the end, we shall prove that $\left(H_{0}\right)$ holds for sufficiently small initial data.

From now on, we assume that $\left(H_{n-1}\right)$ holds. We first follow [2] and show some basic consequences:

Lemma 6. - If $\theta_{0}$ is big enough, then for every $k=0, \ldots, n$, and for every integer $s \in[3, \tilde{\alpha}]$, we have

$$
\begin{aligned}
\left\|\left(V_{k}, \Psi_{k}\right)\right\|_{H_{\gamma}^{s}\left(\Omega_{T}\right)}+\left\|\psi_{k}\right\|_{H_{\gamma}^{s+1}\left(\omega_{T}\right)} & \leq \delta \theta_{k}^{(s-\alpha)_{+}}, \quad \alpha \neq s, \\
\left\|\left(V_{k}, \Psi_{k}\right)\right\|_{H_{\gamma}^{\alpha}\left(\Omega_{T}\right)}+\left\|\psi_{k}\right\|_{H_{\gamma}^{\alpha+1}\left(\omega_{T}\right)} & \leq \delta \log \theta_{k} .
\end{aligned}
$$

The proof follows from the triangle inequality, and from the classical comparisons between series and integrals.

Lemma 7. - If $\theta_{0}$ is big enough, then for every $k=0, \ldots, n$, and for every integer $s \in$ $[3, \tilde{\alpha}+4]$, we have

$$
\begin{aligned}
&\left\|\left(S_{\theta_{k}} V_{k}, S_{\theta_{k}} \Psi_{k}\right)\right\|_{H_{\gamma}^{s}\left(\Omega_{T}\right)} \leq C \delta \theta_{k}^{(s-\alpha)_{+}}, \quad s \neq \alpha, \\
&\left\|\left(S_{\theta_{k}} V_{k}, S_{\theta_{k}} \Psi_{k}\right)\right\|_{H_{\gamma}^{\alpha}\left(\Omega_{T}\right)} \leq C \delta \log \theta_{k} .
\end{aligned}
$$

For every $k=0, \ldots, n$, and for every integer $s \in[3, \tilde{\alpha}]$, we have

$$
\left\|\left(\left(I-S_{\theta_{k}}\right) V_{k},\left(I-S_{\theta_{k}}\right) \Psi_{k}\right)\right\|_{H_{\gamma}^{s}\left(\Omega_{T}\right)} \leq C \delta \theta_{k}^{s-\alpha} .
$$

The proof follows from Lemma 6 and the properties (78) of the smoothing operators.

$4^{\mathrm{e}}$ SÉRIE - TOME $41-2008-\mathrm{N}^{\mathrm{o}} 1$ 


\subsection{Estimate of the quadratic errors}

We start by proving an estimate for the quadratic errors $e_{k}^{\prime}, \hat{e}_{k}^{\prime}, \tilde{e}_{k}^{\prime}$ of the iterative scheme. Recall that these errors are defined by

$$
\begin{aligned}
e_{k}^{\prime} & :=\mathcal{L}\left(V_{k+1}, \Psi_{k+1}\right)-\mathcal{L}\left(V_{k}, \Psi_{k}\right)-\mathcal{L}^{\prime}\left(V_{k}, \Psi_{k}\right)\left(\delta V_{k}, \delta \Psi_{k}\right), \\
\hat{e}_{k}^{\prime} & :=\mathcal{E}\left(V_{k+1}, \Psi_{k+1}\right)-\mathcal{E}\left(V_{k}, \Psi_{k}\right)-\mathcal{E}^{\prime}\left(V_{k}, \Psi_{k}\right)\left(\delta V_{k}, \delta \Psi_{k}\right), \\
\tilde{e}_{k}^{\prime} & :=\mathcal{B}\left(\left(V_{k+1}\right)_{\left.\right|_{x_{2}=0}}, \psi_{k+1}\right)-\mathcal{B}\left(\left(V_{k}\right)_{\left.\right|_{x_{2}=0}}, \psi_{k}\right) \\
& -\mathcal{B}^{\prime}\left(\left(V_{k}\right)_{\left.\right|_{x_{2}=0}}, \psi_{k}\right)\left(\left(\delta V_{k}\right)_{\left.\right|_{x_{2}=0}}, \delta \psi_{k}\right)
\end{aligned}
$$

where $\mathcal{L}, \mathcal{E}$, and $\mathcal{B}$ are defined by (75) and (76).

Lemma 8. - Let $\alpha \geq 4$. There exist $\delta>0$ sufficiently small, and $\theta_{0} \geq 1$ sufficiently large, such that for all $k=0, \ldots, n-1$, and for all integer $s \in[3, \tilde{\alpha}-1]$, one has

$$
\begin{aligned}
&\left\|e_{k}^{\prime}\right\|_{H_{\gamma}^{s}\left(\Omega_{T}\right)} \leq C \delta^{2} \theta_{k}^{L_{1}(s)-1} \Delta_{k}, \\
&\left\|\hat{e}_{k}^{\prime}\right\|_{H_{\gamma}^{s}\left(\Omega_{T}\right)} \leq C \delta^{2} \theta_{k}^{s+1-2 \alpha} \Delta_{k}, \\
&\left\|\tilde{e}_{k}^{\prime}\right\|_{H_{\gamma}^{s}\left(\omega_{T}\right)} \leq C \delta^{2} \theta_{k}^{L_{1}(s)-1} \Delta_{k},
\end{aligned}
$$

where $L_{1}(s):=\max \left\{(s+1-\alpha)_{+}+4-2 \alpha ; s+2-2 \alpha\right\}$.

Proof. - The quadratic error given in (106) may be written as

$$
e_{k}^{\prime}=\int_{0}^{1}(1-\tau) \mathbb{L}^{\prime \prime}\left(U^{a}+V_{k}+\tau \delta V_{k}, \Phi^{a}+\Psi_{k}+\tau \delta \Psi_{k}\right)\left(\left(\delta V_{k}, \delta \Psi_{k}\right),\left(\delta V_{k}, \delta \Psi_{k}\right)\right) d \tau .
$$

From Theorem 11, (102) and (103a), we have

$$
\sup _{\tau \in[0,1]}\left\|\left(\dot{U}^{a}+V_{k}+\tau \delta V_{k}, \dot{\Phi}^{a}+\Psi_{k}+\tau \delta \Psi_{k}\right)\right\|_{W^{1, \infty}\left(\Omega_{T}\right)} \leq C \delta,
$$

so for $\delta$ sufficiently small, we can apply Proposition 5. Using $\left(H_{n-1}\right),(102)$ and (103) we obtain (109a). The estimate (109b) of $\hat{e}_{k}^{\prime}$ is similar, and follows from (98). The quadratic error $\tilde{e}_{k}^{\prime}$ is estimated by means of (99), a classical trace estimate and the Sobolev imbedding Theorem.

\subsection{Estimate of the first substitution errors}

Now we estimate the first substitution errors $e_{k}^{\prime \prime}, \hat{e}_{k}^{\prime \prime}, \tilde{e}_{k}^{\prime \prime}$ of the iterative scheme, defined by (110) $e_{k}^{\prime \prime}:=\mathcal{L}^{\prime}\left(V_{k}, \Psi_{k}\right)\left(\delta V_{k}, \delta \Psi_{k}\right)-\mathcal{L}^{\prime}\left(S_{\theta_{k}} V_{k}, S_{\theta_{k}} \Psi_{k}\right)\left(\delta V_{k}, \delta \Psi_{k}\right)$,

(111) $\hat{e}_{k}^{\prime \prime}:=\mathcal{E}^{\prime}\left(V_{k}, \Psi_{k}\right)\left(\delta V_{k}, \delta \Psi_{k}\right)-\mathcal{E}^{\prime}\left(S_{\theta_{k}} V_{k}, S_{\theta_{k}} \Psi_{k}\right)\left(\delta V_{k}, \delta \Psi_{k}\right)$,

(112) $\quad \tilde{e}_{k}^{\prime \prime}:=\mathcal{B}^{\prime}\left(\left(V_{k}\right)_{\left.\right|_{x_{2}=0}}, \psi_{k}\right)\left(\left(\delta V_{k}\right)_{\left.\right|_{x_{2}=0}}, \delta \psi_{k}\right)-\mathcal{B}^{\prime}\left(\left(S_{\theta_{k}} V_{k}\right)_{\left.\right|_{x_{2}=0}}, \psi_{k}^{\sharp}\right)\left(\left(\delta V_{k}\right)_{\left.\right|_{x_{2}=0}, \delta \psi_{k}}\right)$,

where $\psi_{k}^{\sharp}$ denotes the common trace of $S_{\theta_{k}} \Psi_{k}^{ \pm}$on the boundary $\omega_{T}$. (Recall that these traces coincide thanks to the properties of the smoothing operators.) 
Lemma 9. - Let $\alpha \geq 4$. There exist $\delta>0$ sufficiently small, and $\theta_{0} \geq 1$ sufficiently large, such that for all $k=0, \ldots, n-1$, and for all integer $s \in[3, \tilde{\alpha}-2]$, one has

$$
\begin{aligned}
\left\|e_{k}^{\prime \prime}\right\|_{H_{\gamma}^{s}\left(\Omega_{T}\right)} & \leq C \delta^{2} \theta_{k}^{L_{2}(s)-1} \Delta_{k}, \\
\left\|\hat{e}_{k}^{\prime \prime}\right\|_{H_{\gamma}^{s}\left(\Omega_{T}\right)} & \leq C \delta^{2} \theta_{k}^{s+3-2 \alpha} \Delta_{k}, \\
\left\|\tilde{e}_{k}^{\prime \prime}\right\|_{H_{\gamma}^{s}\left(\omega_{T}\right)} & \leq C \delta^{2} \theta_{k}^{L_{2}(s)-1} \Delta_{k},
\end{aligned}
$$

where $L_{2}(s):=\max \left\{(s+1-\alpha)_{+}+6-2 \alpha ; s+5-2 \alpha\right\}$.

Proof. - The substitution error given in (110) may be written as

$$
\begin{array}{r}
e_{k}^{\prime \prime}=\int_{0}^{1} \mathbb{L}^{\prime \prime}\left(U^{a}+S_{\theta_{k}} V_{k}+\tau\left(I-S_{\theta_{k}}\right) V_{k}, \Phi^{a}+S_{\theta_{k}} \Psi_{k}+\tau\left(I-S_{\theta_{k}}\right) \Psi_{k}\right) \\
\left(\left(\delta V_{k}, \delta \Psi_{k}\right),\left(\left(I-S_{\theta_{k}}\right) V_{k},\left(I-S_{\theta_{k}}\right) \Psi_{k}\right)\right) d \tau .
\end{array}
$$

We first show that we can apply Proposition 5 for $\delta$ sufficiently small. For $s+1 \neq \alpha$ and $s+1 \leq \tilde{\alpha}$, the estimate (113a) follows from (102), $\left(H_{n-1}\right),(104 \mathrm{a})$ and (105). For $s+1=\alpha$, the proof requires the use of (104b). (113b) follows in the same way. The substitution error given in (112) is estimated by using (99), $\left(H_{n-1}\right)$ and (105).

\subsection{Construction and estimate of the modified state}

The next step requires the construction of the smooth modified state $V_{n+1 / 2}, \Psi_{n+1 / 2}$, $\psi_{n+1 / 2}$ satisfying the constraints (16) and (17). The additional constraint (18) will be obtained by choosing $\delta$ small enough, so we first focus on (16), (17).

Proposition 7. - Let $\alpha \geq 4$. There exist some functions $V_{n+1 / 2}, \Psi_{n+1 / 2}, \psi_{n+1 / 2}$, that vanish in the past, and such that $U^{a}+V_{n+1 / 2}, \Phi^{a}+\Psi_{n+1 / 2}, \varphi^{a}+\psi_{n+1 / 2}$ satisfy the constraints (16) and (17). Moreover, these functions satisfy:

$$
\begin{aligned}
& \Psi_{n+1 / 2}^{ \pm}=S_{\theta_{n}} \Psi_{n}^{ \pm}, \quad \psi_{n+1 / 2}:=\left(S_{\theta_{n}} \Psi_{n}^{ \pm}\right)_{\left.\right|_{x_{2}}=0} \\
& v_{n+1 / 2}^{ \pm}=S_{\theta_{n}} v_{n}^{ \pm}, \\
& \left\|V_{n+1 / 2}-S_{\theta_{n}} V_{n}\right\|_{H_{\gamma}^{s}\left(\Omega_{T}\right)} \leq C \delta \theta_{n}^{s+1-\alpha}, \quad \text { for } s \in[3, \tilde{\alpha}+3] .
\end{aligned}
$$

Proof. - We want to construct some functions $V_{n+1 / 2}, \Psi_{n+1 / 2}, \psi_{n+1 / 2}$ that satisfy

$$
\begin{aligned}
& \left(\Psi_{n+1 / 2}^{+}\right)_{\left.\right|_{x_{2}=0}}=\left(\Psi_{n+1 / 2}^{-}\right)_{\left.\right|_{x_{2}=0}}=\psi_{n+1 / 2}, \\
& \mathbb{B}\left(\left(U^{a}+V_{n+1 / 2}\right)_{\left.\right|_{x_{2}=0}}, \varphi^{a}+\psi_{n+1 / 2}\right)=0, \\
& \mathcal{E}\left(V_{n+1 / 2}, \Psi_{n+1 / 2}\right)=0 .
\end{aligned}
$$

We note that the eikonal equations on the boundary, that is the first two components of the operator $\mathbb{B}$, can be deduced from the eikonal equations in the interior. In other words, it is enough to construct some functions that satisfy:

$$
\begin{aligned}
& \left(\Psi_{n+1 / 2}^{+}\right)_{\left.\right|_{x_{2}=0}}=\left(\Psi_{n+1 / 2}^{-}\right)_{\left.\right|_{x_{2}=0}}=\psi_{n+1 / 2} \\
& \left(\rho_{n+1 / 2}\right)_{\left.\right|_{x_{2}=0}}^{+}=\left(\rho_{n+1 / 2}\right)_{\left.\right|_{x_{2}=0}}^{-}, \\
& \mathcal{E}\left(V_{n+1 / 2}, \Psi_{n+1 / 2}\right)=0 .
\end{aligned}
$$


Let the errors $\varepsilon_{1,2}^{n}$ be defined by

$$
\begin{aligned}
& \varepsilon_{1}^{n}:=\left(S_{\theta_{n}} \rho_{n}^{+}\right)_{\left.\right|_{x_{2}=0}}-\left(S_{\theta_{n}} \rho_{n}^{-}\right)_{\left.\right|_{x_{2}=0}}, \\
& \varepsilon_{2}^{n}:=\mathcal{E}\left(V_{n}, \Psi_{n}\right) .
\end{aligned}
$$

We define the modified states $V_{n+1 / 2}, \Psi_{n+1 / 2}, \psi_{n+1 / 2}$ as follows:

$$
\begin{gathered}
\Psi_{n+1 / 2}^{ \pm}:=S_{\theta_{n}} \Psi_{n}^{ \pm}, \quad \rho_{n+1 / 2}^{ \pm}:=S_{\theta_{n}} \rho_{n}^{ \pm} \mp \frac{1}{2} \mathcal{R}_{T} \varepsilon_{1}^{n}, \quad v_{n+1 / 2}^{ \pm}:=S_{\theta_{n}} v_{n}^{ \pm}, \\
u_{n+1 / 2}^{ \pm}:=\partial_{t} \Psi_{n+1 / 2}^{ \pm}+\left(v^{a, \pm}+v_{n+1 / 2}^{ \pm}\right) \partial_{x_{1}} \Psi_{n+1 / 2}^{ \pm}+v_{n+1 / 2}^{ \pm} \partial_{x_{1}} \Phi^{a}
\end{gathered}
$$

where $\mathcal{R}_{T}$ is the lifting operator introduced in Lemma 5. It is easy to check that $V_{n+1 / 2}$, $\Psi_{n+1 / 2}, \psi_{n+1 / 2}$ fulfill (115) and vanish in the past. We thus only need to prove the estimate (114c).

First of all, we note that points a) and c) of the induction assumption $\left(H_{n-1}\right)$ yield

$$
\begin{aligned}
\|\left(\rho_{n}^{+}-\rho_{n}^{-}\right)_{\left.\right|_{x_{2}=0}} & \left\|_{H_{\gamma}^{s}\left(\omega_{T}\right)} \leq\right\|\left(\rho_{n-1}^{+}-\rho_{n-1}^{-}\right)_{\left.\right|_{x_{2}=0}}\left\|_{H_{\gamma}^{s}\left(\omega_{T}\right)}+\right\|\left(\delta \rho_{n-1}^{+}-\delta \rho_{n-1}^{-}\right)_{\left.\right|_{x_{2}=0}} \|_{H_{\gamma}^{s}\left(\omega_{T}\right)} \\
& \leq\left\|\mathcal{B}\left(\left(V_{n-1}\right)_{\left.\right|_{x_{2}=0}}, \psi_{n-1}\right)\right\|_{H_{\gamma}^{s}\left(\omega_{T}\right)}+C\left\|\delta V_{n-1}\right\|_{H_{\gamma}^{s+1}\left(\Omega_{T}\right)} \leq C \delta \theta_{n}^{s-\alpha-1},
\end{aligned}
$$

for all integer $s \in[4, \alpha]$. Using the properties of the operators $\mathcal{R}_{T}$ and $S_{\theta}$ yields

$$
\left\|\varepsilon_{1}^{n}\right\|_{H_{\gamma}^{s}\left(\omega_{T}\right)} \leq C \theta_{n}^{s+1-\alpha}\left\|\left(\rho_{n}^{+}-\rho_{n}^{-}\right)_{\left.\right|_{x_{2}=0}}\right\|_{H_{\gamma}^{\alpha}\left(\omega_{T}\right)} \leq C \delta \theta_{n}^{s-\alpha}
$$

for $s \in[\alpha, \tilde{\alpha}+3]$, while for $s \in[3, \alpha-1]$, one has

$$
\left\|\varepsilon_{1}^{n}\right\|_{H_{\gamma}^{s}\left(\omega_{T}\right)} \leq C\left\|\left(\rho_{n}^{+}-\rho_{n}^{-}\right)_{\left.\right|_{x_{2}=0}}\right\|_{H_{\gamma}^{s+1}\left(\omega_{T}\right)} \leq C \delta \theta_{n}^{s-\alpha} .
$$

For $s \in[3, \tilde{\alpha}+3]$, we thus obtain

$$
\left\|\rho_{n+1 / 2}^{ \pm}-S_{\theta_{n}} \rho_{n}^{ \pm}\right\|_{H_{\gamma}^{s}\left(\Omega_{T}\right)}=\frac{1}{2}\left\|\mathcal{R}_{T} \varepsilon_{1}^{n}\right\|_{H_{\gamma}^{s}\left(\Omega_{T}\right)} \leq C\left\|\varepsilon_{1}^{n}\right\|_{H_{\gamma}^{s}\left(\omega_{T}\right)} \leq C \delta \theta_{n}^{s-\alpha} .
$$

We now turn to the estimate of $u_{n+1 / 2}-S_{\theta_{n}} u_{n}$. Using the definition (116b) of $\varepsilon_{2}^{n}$, we get

$$
S_{\theta_{n}} u_{n}+S_{\theta_{n}} \varepsilon_{2}^{n}=S_{\theta_{n}} \partial_{t} \Psi_{n}+S_{\theta_{n}}\left(\left(v^{a}+v_{n}\right) \partial_{x_{1}} \Psi_{n}\right)+S_{\theta_{n}}\left(v_{n} \partial_{x_{1}} \Phi^{a}\right) .
$$

Combining with the definition of $u_{n+1 / 2}$, see (117), we can thus compute the relation

$$
+\left[\left(\dot{v}^{a}+S_{\theta_{n}} v_{n}\right) \partial_{x_{1}} S_{\theta_{n}} \Psi_{n}-S_{\theta_{n}}\left(\left(\dot{v}^{a}+v_{n}\right) \partial_{x_{1}} \Psi_{n}\right)\right]+\left(S_{\theta_{n}} v_{n}\right) \partial_{x_{1}} \Phi^{a}-S_{\theta_{n}}\left(v_{n} \partial_{x_{1}} \Phi^{a}\right) .
$$

To estimate the first term $S_{\theta_{n}} \varepsilon_{2}^{n}$ on the right-hand side we use the decomposition:

$\varepsilon_{2}^{n}=\mathcal{E}\left(V_{n-1}, \Psi_{n-1}\right)+\partial_{t}\left(\delta \Psi_{n-1}\right)+\left(v^{a}+v_{n-1}\right) \partial_{x_{1}}\left(\delta \Psi_{n-1}\right)+\delta v_{n-1} \partial_{x_{1}}\left(\Phi^{a}+\Psi_{n}\right)-\delta u_{n-1}$, then exploit point $\mathrm{d})$ of $\left(H_{n-1}\right)$ and the properties of the smoothing operators. We detail now the estimate of the most involved commutator in (118). Assume first that $s \in[\alpha+1, \tilde{\alpha}+3]$. Then we have

$$
\begin{gathered}
\left\|\left(\dot{v}^{a}+S_{\theta_{n}} v_{n}\right) \partial_{x_{1}} S_{\theta_{n}} \Psi_{n}\right\|_{H_{\gamma}^{s}\left(\Omega_{T}\right)} \leq C\left\|\dot{v}^{a}+S_{\theta_{n}} v_{n}\right\|_{L^{\infty}\left(\Omega_{T}\right)}\left\|S_{\theta_{n}} \Psi_{n}\right\|_{H_{\gamma}^{s+1}\left(\Omega_{T}\right)} \\
+C\left\|S_{\theta_{n}} \Psi_{n}\right\|_{W^{1, \infty}\left(\Omega_{T}\right)}\left\|\dot{v}^{a}+S_{\theta_{n}} v_{n}\right\|_{H_{\gamma}^{s}\left(\Omega_{T}\right)} \leq C \delta^{2} \theta_{n}^{s+1-\alpha}, \\
\left\|S_{\theta_{n}}\left(\left(\dot{v}^{a}+v_{n}\right) \partial_{x_{1}} \Psi_{n}\right)\right\|_{H_{\gamma}^{s}\left(\Omega_{T}\right)} \leq C \theta_{n}^{s-\alpha}\left\|\left(\dot{v}^{a}+v_{n}\right) \partial_{x_{1}} \Psi_{n}\right\|_{H_{\gamma}^{\alpha}\left(\Omega_{T}\right)} \leq C \delta^{2} \theta_{n}^{s+1-\alpha} .
\end{gathered}
$$


These two inequalities give (114c) when $s \in[\alpha+1, \tilde{\alpha}+3]$. In order to prove that this estimate is also valid for $s \in[3, \alpha]$, we use the triangle inequality:

$$
\begin{aligned}
\|\left(\dot{v}^{a}+\right. & \left.S_{\theta_{n}} v_{n}\right) \partial_{x_{1}} S_{\theta_{n}} \Psi_{n}-S_{\theta_{n}}\left(\left(\dot{v}^{a}+v_{n}\right) \partial_{x_{1}} \Psi_{n}\right) \|_{H_{\gamma}^{s}\left(\Omega_{T}\right)} \\
\leq & \left\|\left(v_{n}-S_{\theta_{n}} v_{n}\right) \partial_{x_{1}} S_{\theta_{n}} \Psi_{n}\right\|_{H_{\gamma}^{s}\left(\Omega_{T}\right)}+\left\|\left(\dot{v}^{a}+v_{n}\right) \partial_{x_{1}}\left(\Psi_{n}-S_{\theta_{n}} \Psi_{n}\right)\right\|_{H_{\gamma}^{s}\left(\Omega_{T}\right)} \\
& +\left\|\left(I-S_{\theta_{n}}\right)\left(\left(\dot{v}^{a}+v_{n}\right) \partial_{x_{1}} \Psi_{n}\right)\right\|_{H_{\gamma}^{s}\left(\Omega_{T}\right)} .
\end{aligned}
$$

For each term of the right-hand side, we use the properties of the smoothing operators, as well as the classical tame estimate for a product. The details are similar to what was done before, so we omit them. Applying the same strategy to the other commutators in (118), we can complete the proof of (114c).

\subsection{Estimate of the second substitution errors}

Now we may estimate the second substitution errors $e_{k}^{\prime \prime \prime}, \hat{e}_{k}^{\prime \prime \prime}$, and $\tilde{e}_{k}^{\prime \prime \prime}$ of the iterative scheme, that are defined by (recall that $\psi_{k}^{\sharp}$ denotes the common trace of $S_{\theta_{k}} \Psi_{k}^{ \pm}$on the boundary $\omega_{T}$ ):

$$
\begin{aligned}
e_{k}^{\prime \prime \prime}:= & \mathcal{L}^{\prime}\left(S_{\theta_{k}} V_{k}, S_{\theta_{k}} \Psi_{k}\right)\left(\delta V_{k}, \delta \Psi_{k}\right)-\mathcal{L}^{\prime}\left(V_{k+1 / 2}, \Psi_{k+1 / 2}\right)\left(\delta V_{k}, \delta \Psi_{k}\right), \\
\hat{e}_{k}^{\prime \prime \prime}:= & \mathcal{E}^{\prime}\left(S_{\theta_{k}} V_{k}, S_{\theta_{k}} \Psi_{k}\right)\left(\delta V_{k}, \delta \Psi_{k}\right)-\mathcal{E}^{\prime}\left(V_{k+1 / 2}, \Psi_{k+1 / 2}\right)\left(\delta V_{k}, \delta \Psi_{k}\right), \\
\tilde{e}_{k}^{\prime \prime \prime}:= & \mathcal{B}^{\prime}\left(\left(S_{\theta_{k}} V_{k}\right)_{\left.\right|_{x_{2}=0}}, \psi_{k}^{\sharp}\right)\left(\left(\delta V_{k}\right)_{\left.\right|_{x_{2}=0}}, \delta \psi_{k}\right) \\
& -\mathcal{B}^{\prime}\left(\left(V_{k+1 / 2}\right)_{\left.\right|_{x_{2}=0}}, \psi_{k+1 / 2}\right)\left(\left(\delta V_{k}\right)_{\left.\right|_{x_{2}=0}}, \delta \psi_{k}\right) .
\end{aligned}
$$

Lemma 10. - Let $\alpha \geq 4$. There exist $\delta>0$ sufficiently small and $\theta_{0} \geq 1$ sufficiently large such that, for all $k=0, \ldots, n-1$, and for all integer $s \in[3, \tilde{\alpha}-1]$, one has $\hat{e}_{k}^{\prime \prime \prime}=\tilde{e}_{k}^{\prime \prime \prime}=0$, and

$$
\left\|e_{k}^{\prime \prime \prime}\right\|_{H_{\gamma}\left(\Omega_{T}\right)} \leq C \delta^{2} \theta_{k}^{L_{3}(s)-1} \Delta_{k},
$$

where $L_{3}(s):=\max \left\{(s+1-\alpha)_{+}+8-2 \alpha ; s+5-2 \alpha\right\}$.

Proof. - Using the expression (114a), the substitution error given in (119a) may be written as

$$
\begin{aligned}
e_{k}^{\prime \prime \prime}=\int_{0}^{1} \mathbb{L}^{\prime \prime}\left(U^{a}+V_{k+1 / 2}+\tau\left(S_{\theta_{k}} V_{k}-V_{k+1 / 2}\right),\right. & \left.\Phi^{a}+S_{\theta_{k}} \Psi_{k}\right) \\
& \left(\left(\delta V_{k}, \delta \Psi_{k}\right),\left(S_{\theta_{k}} V_{k}-V_{k+1 / 2}, 0\right)\right) d \tau .
\end{aligned}
$$

Using Lemma 7, and Proposition 7, we first derive the bound

$$
\begin{array}{r}
\left\|\left(\dot{U}^{a}+V_{k+1 / 2}+\tau\left(S_{\theta_{k}} V_{k}-V_{k+1 / 2}\right), \dot{\Phi}^{a}+S_{\theta_{k}} \Psi_{k}\right)\right\|_{H_{\gamma}^{s+1}\left(\Omega_{T}\right)} \leq C \delta \theta_{k}^{(s+1-\alpha)_{+}+1}, \\
s \in[3, \tilde{\alpha}-1] .
\end{array}
$$

Then (120) follows by applying a Sobolev imbedding Theorem, Proposition 5, $\left(H_{n-1}\right)$ and Proposition 7, provided that $\delta$ is small enough. We write the substitution error given in (119c) as

$$
\tilde{e}_{k}^{\prime \prime \prime}=\mathbb{B}^{\prime \prime}\left(\left(\left(\delta V_{k}\right)_{\left.\right|_{x_{2}=0}}, \delta \psi_{k}\right),\left(\left(S_{\theta_{k}} V_{k}-V_{k+1 / 2}\right)_{\left.\left.\left.\right|_{x_{2}=0}, 0\right)\right)} .\right.\right.
$$

Using the exact expression of $\mathbb{B}^{\prime \prime}$ and the equality $v_{k+1 / 2}=S_{\theta_{k}} v_{k}$, we get $\tilde{e}_{k}^{\prime \prime \prime}=0$. The same argument applies to $\hat{e}_{k}^{\prime \prime \prime}$. 


\subsection{Estimate of the last error term}

In our iterative scheme we have a last error term to be estimated, namely

$$
D_{k+1 / 2} \delta \Psi_{k}:=\frac{\delta \Psi_{k}}{\partial_{x_{2}}\left(\Phi^{a}+\Psi_{k+1 / 2}\right)} \partial_{x_{2}}\left\{\mathbb{L}\left(U^{a}+V_{k+1 / 2}, \Phi^{a}+\Psi_{k+1 / 2}\right)\right\}
$$

which results from the introduction of the good unknown in the decomposition of the linearized equations, see (82). Let us set

$$
R_{k}:=\partial_{x_{2}}\left\{\mathbb{L}\left(U^{a}+V_{k+1 / 2}, \Phi^{a}+\Psi_{k+1 / 2}\right)\right\} .
$$

Since $V_{k+1 / 2}$ and $\Psi_{k+1 / 2}$ vanish in the past, $R_{k}$ does not vanish in the past. However, $\delta \Psi_{k}$ vanishes in the past, so the error term $D_{k+1 / 2} \delta \Psi_{k}$ also vanishes in the past.

From (102), (104a) and (114a), we have $\left|\partial_{x_{2}}\left(\Phi^{a}+\Psi_{k+1 / 2}\right)\right| \geq 1 / 2$, provided that $\delta$ is small enough. Then Theorem 9 enables us to obtain:

$$
\begin{array}{r}
\left\|D_{k+1 / 2} \delta \Psi_{k}\right\|_{H_{\gamma}^{s}\left(\Omega_{T}\right)}=\left\|D_{k+1 / 2} \delta \Psi_{k}\right\|_{H_{\gamma}^{s}\left(\Omega_{T}^{+}\right)} \leq C\left\{\left\|\delta \Psi_{k}\right\|_{H_{\gamma}^{s}\left(\Omega_{T}^{+}\right)}\left\|R_{k}\right\|_{L^{\infty}\left(\Omega_{T}^{+}\right)}\right. \\
\left.+\left\|\delta \Psi_{k}\right\|_{L^{\infty}\left(\Omega_{T}^{+}\right)}\left(\left\|R_{k}\right\|_{H_{\gamma}^{s}\left(\Omega_{T}^{+}\right)}+\left\|R_{k}\right\|_{L^{\infty}\left(\Omega_{T}^{+}\right)}\left\|\dot{\Phi}^{a}+\Psi_{k+1 / 2}\right\|_{H_{\gamma}^{s+1}\left(\Omega_{T}^{+}\right)}\right)\right\}
\end{array}
$$

Lemma 11. - Let $\alpha \geq 4$, $\tilde{\alpha} \geq \alpha+2$. For $\delta>0$ sufficiently small, $\theta_{0} \geq 1$ sufficiently large, for all $k=0, \ldots, n-1$, and for all integer $s \in[3, \tilde{\alpha}-2]$, one has

$$
\left\|R_{k}\right\|_{H_{\gamma}^{s}\left(\Omega_{T}^{+}\right)} \leq C \delta\left(\theta_{k}^{s+3-\alpha}+\theta_{k}^{(s+2-\alpha)_{+}+5-\alpha}\right) .
$$

Proof. - We proceed as in [1]. If $s \leq \tilde{\alpha}-3$, we introduce the following decomposition:

$$
\begin{aligned}
\mathbb{L}\left(U^{a}+V_{k+1 / 2}, \Phi^{a}+\Psi_{k+1 / 2}\right)= & \mathbb{L}\left(U^{a}+V_{k+1 / 2}, \Phi^{a}+\Psi_{k+1 / 2}\right) \\
& -\mathbb{L}\left(U^{a}+V_{k}, \Phi^{a}+\Psi_{k}\right)+\mathcal{L}\left(V_{k}, \Psi_{k}\right)-f^{a} .
\end{aligned}
$$

Then the estimate follows from the induction assumption $\left(H_{n-1}\right)$, Proposition 7 , and Lemma 7. If $s=\tilde{\alpha}-2$ the estimate is obtained directly from (121).

We are ready to prove the estimate of the last error term:

Lemma 12. - Let $\alpha \geq 5$, $\tilde{\alpha} \geq \alpha+2$. There exist $\delta>0$ sufficiently small and $\theta_{0} \geq 1$ sufficiently large such that, for all $k=0, \ldots, n-1$, and for all integer $s \in[3, \tilde{\alpha}-2]$, one has

$$
\left\|D_{k+1 / 2} \delta \Psi_{k}\right\|_{H_{\gamma}^{s}\left(\Omega_{T}\right)} \leq C \delta^{2} \theta_{k}^{L(s)-1} \Delta_{k},
$$

where $L(s):=\max \left\{(s+2-\alpha)_{+}+8-2 \alpha ;(s+1-\alpha)_{+}+9-2 \alpha ; s+6-2 \alpha\right\}$.

Proof. - We first use Lemma 11 to derive the bound $\left\|R_{k}\right\|_{L^{\infty}\left(\Omega_{T}^{+}\right)} \leq C \delta \theta_{k}^{6-\alpha}$. We combine this $L^{\infty}$ bound and (123) in (122). The terms in $\delta \Psi_{k}$ are estimated by the induction assumption $\left(H_{n-1}\right)$, and the terms in $\Psi_{k+1 / 2}=S_{\theta_{k}} \Psi_{k}$ are estimated by Lemma 7 . Putting all these estimates together yields (125). 


\subsection{Convergence of the iteration scheme}

We first estimate the errors $e_{k}, \hat{e}_{k}$ and $\tilde{e}_{k}$ :

Lemma 13. - Let $\alpha \geq 5$. There exist $\delta>0$ sufficiently small, and $\theta_{0} \geq 1$ sufficiently large, such that for all $k=0, \ldots, n-1$ and all integer $s \in[3, \tilde{\alpha}-2]$, one has

$$
\left\|e_{k}\right\|_{H_{\gamma}^{s}\left(\Omega_{T}\right)}+\left\|\tilde{e}_{k}\right\|_{H_{\gamma}^{s}\left(\omega_{T}\right)} \leq C \delta^{2} \theta_{k}^{L(s)-1} \Delta_{k},\left\|\hat{e}_{k}\right\|_{H_{\gamma}^{s}\left(\Omega_{T}\right)} \leq C \delta^{2} \theta_{k}^{s+3-2 \alpha} \Delta_{k},
$$

where $L(s)$ is defined in Lemma 12.

Proof. - We recall that $e_{k}, \hat{e}_{k}, \tilde{e}_{k}$ are defined in (84) as the sum of all the error terms of the $k$-th step. Adding the estimates (109), (113), (120) and (125), we obtain (126).

The preceding lemma immediately yields the estimate of the accumulated errors $E_{n}$, and $\tilde{E}_{n}$ :

Lemma 14. - Let $\alpha \geq 7, \tilde{\alpha}=\alpha+4$ and $r=\alpha+1$. There exist $\delta>0$ sufficiently small, $\theta_{0} \geq 1$ sufficiently large, such that

$$
\left\|E_{n}\right\|_{H_{\gamma}^{r+1}\left(\Omega_{T}\right)}+\left\|\tilde{E}_{n}\right\|_{H_{\gamma}^{r+1}\left(\omega_{T}\right)} \leq C \delta^{2} \theta_{n}, \quad\left\|\hat{E}_{n}\right\|_{H_{\gamma}^{r+1}\left(\Omega_{T}\right)} \leq \delta^{2} .
$$

Going on with the iteration scheme, the next lemma gives the estimates of the source terms $f_{n}, g_{n}, h_{n}^{ \pm}$, defined by equations (86), (93) and (94):

Lemma 15. - Let $\alpha \geq 7$, and let $\tilde{\alpha}, r$ be given as in Lemma 14. There exist $\delta>0$ sufficiently small and $\theta_{0} \geq 1$ sufficiently large, such that for all integer $s \in[3, \tilde{\alpha}+1]$, one has

$$
\begin{aligned}
& \left\|f_{n}\right\|_{H_{\gamma}^{s}\left(\Omega_{T}\right)} \leq C \Delta_{n}\left\{\theta_{n}^{s-\alpha-2}\left(\left\|f^{a}\right\|_{H_{\gamma}^{\alpha+1}\left(\Omega_{T}\right)}+\delta^{2}\right)+\delta^{2} \theta_{n}^{L(s)-1}\right\}, \\
& \left\|g_{n}\right\|_{H_{\gamma}^{s}\left(\omega_{T}\right)} \leq C \delta^{2} \Delta_{n}\left(\theta_{n}^{L(s)-1}+\theta_{n}^{s-\alpha-2}\right),
\end{aligned}
$$

and for all integer $s \in[3, \tilde{\alpha}]$, one has

$$
\left\|h_{n}\right\|_{H_{\gamma}^{s}\left(\Omega_{T}\right)} \leq C \delta^{2} \Delta_{n}\left(\theta_{n}^{L(s)-1}+\theta_{n}^{s-\alpha-2}\right) .
$$

Proof. - From (86) we have

$$
f_{n}=\left(S_{\theta_{n}}-S_{\theta_{n-1}}\right) f^{a}-\left(S_{\theta_{n}}-S_{\theta_{n-1}}\right) E_{n-1}-S_{\theta_{n}} e_{n-1} .
$$

Using (78), (126) and (127) gives (128a), with $\Delta_{n-1}, \theta_{n-1}$ instead of $\Delta_{n}, \theta_{n}$. Using $\theta_{n-1} \leq$ $\theta_{n} \leq \sqrt{2} \theta_{n-1}$ and $\Delta_{n-1} \leq 3 \Delta_{n}$ yields (128a). Estimate (128b) follows in the same way. To prove (129), we use (93) to derive:

$$
h_{n}^{+}=\left(S_{\theta_{n}}-S_{\theta_{n-1}}\right)\left(\mathcal{R}_{T} \tilde{E}_{n-1,2}-\hat{E}_{n-1}^{+}\right)+S_{\theta_{n}}\left(\mathcal{R}_{T} \tilde{e}_{n-1,2}-\hat{e}_{n-1}^{+}\right) .
$$

Then we use (126) and (127) as above. The estimate of $h_{n}^{-}$is the same.

We now consider problem (87), that gives the solution $\left(\delta \dot{V}_{n}, \delta \psi_{n}\right)$. Then we find $\Psi_{n+1}^{ \pm}$, and consequently $\left(\delta V_{n}, \delta \Psi_{n}\right)$ :

Lemma 16. - Assume $\alpha \geq 7$. If $\delta>0$ and $\left\|f^{a}\right\|_{H_{\gamma}^{\alpha+1}\left(\Omega_{T}\right)} / \delta$ are sufficiently small, $\theta_{0} \geq 1$ is sufficiently large, then for all $3 \leq s \leq \tilde{\alpha}$, one has

$$
\left\|\left(\delta V_{n}, \delta \Psi_{n}\right)\right\|_{H_{\gamma}^{s}\left(\Omega_{T}\right)}+\left\|\delta \psi_{n}\right\|_{H_{\gamma}^{s+1}\left(\omega_{T}\right)} \leq \delta \theta_{n}^{s-\alpha-1} \Delta_{n} .
$$


Proof. - Let us consider problem (87), which may be solved because $U^{a}+V_{n+1 / 2}, \Phi^{a}+$ $\Psi_{n+1 / 2}$ satisfy the required constraints, in particular (16) and (17). Note that the constraint (15) can be obtained by truncating the coefficients $\left(V_{n+1 / 2}, \Psi_{n+1 / 2}, \psi_{n+1 / 2}\right)$ by a suitable cut-off function. This truncation does not affect the coefficients on the set $\left\{t \in[0, T], x_{2} \geq 0\right.$, $\left.\sqrt{x_{1}^{2}+x_{2}^{2}} \leq 4\right\}$. We can thus consider coefficients with a fixed compact support. In order to apply Proposition 6, we first verify (100), by means of the classical trace estimate, (102), (104a), (114) and taking $\delta$ sufficiently small (note that we use $\alpha \geq 7$ ). Thus we may apply estimate (101) in order to obtain

$$
\begin{aligned}
& \left\|\delta \dot{V}_{n}\right\|_{H_{\gamma}^{s}\left(\Omega_{T}\right)}+\left\|\delta \psi_{n}\right\|_{H_{\gamma}^{s+1}\left(\omega_{T}\right)} \leq C\left\{\left\|f_{n}\right\|_{H_{\gamma}^{s+1}\left(\Omega_{T}\right)}+\left\|g_{n}\right\|_{H_{\gamma}^{s+1}\left(\omega_{T}\right)}\right. \\
& \left.\quad+\left(\left\|f_{n}\right\|_{H_{\gamma}^{4}\left(\Omega_{T}\right)}+\left\|g_{n}\right\|_{H_{\gamma}^{4}\left(\omega_{T}\right)}\right)\left\|\left(\dot{U}^{a}+V_{n+1 / 2}, \dot{\Phi}^{a}+\Psi_{n+1 / 2}\right)\right\|_{H_{\gamma}^{s+3}\left(\Omega_{T}\right)}\right\} .
\end{aligned}
$$

The particular case $s=3$ yields

$$
\left\|\delta \dot{V}_{n}\right\|_{H_{\gamma}^{3}\left(\Omega_{T}\right)} \leq C\left(\left\|f_{n}\right\|_{H_{\gamma}^{4}\left(\Omega_{T}\right)}+\left\|g_{n}\right\|_{H_{\gamma}^{4}\left(\omega_{T}\right)}\right) .
$$

Given $\delta \psi_{n}$, we determine $\delta \Psi_{n}$ from the equations (90) and (91). We can perform energy estimates for $\delta \Psi_{n}^{ \pm}$, by following what was done earlier for the vorticity equation. For simplicity, we drop the \pm subscripts. Using Lemma 7, Proposition 7, together with Sobolev's imbedding Theorem, we obtain the estimate

$$
\begin{aligned}
& \gamma\left\|\delta \Psi_{n}\right\|_{H_{\gamma}^{s}\left(\Omega_{T}\right)} \leq C\left\{\left\|g_{n}\right\|_{H_{\gamma}^{s}\left(\omega_{T}\right)}+\left\|h_{n}\right\|_{H_{\gamma}^{s}\left(\Omega_{T}\right)}+\left\|\delta \dot{V}_{n}\right\|_{H_{\gamma}^{s}\left(\Omega_{T}\right)}\right. \\
& \left.\quad+\left\|\delta \dot{V}_{n}\right\|_{H_{\gamma}^{3}\left(\Omega_{T}\right)}\left\|\Phi^{a}+S_{\theta_{n}} \Psi_{n}\right\|_{H_{\gamma}^{s+1}\left(\Omega_{T}\right)}+\delta \theta_{n}^{(s+2-\alpha)_{+}}\left\|\delta \Psi_{n}\right\|_{H_{\gamma}^{3}\left(\Omega_{T}\right)}\right\}
\end{aligned}
$$

for all integer $s \in[3, \tilde{\alpha}]$ and $\delta$ small enough. Choosing $s=3$, and using (132), we obtain

$$
\left\|\delta \Psi_{n}\right\|_{H_{\gamma}^{3}\left(\Omega_{T}\right)} \leq C\left(\left\|f_{n}\right\|_{H_{\gamma}^{4}\left(\Omega_{T}\right)}+\left\|h_{n}\right\|_{H_{\gamma}^{3}\left(\Omega_{T}\right)}+\left\|g_{n}\right\|_{H_{\gamma}^{4}\left(\omega_{T}\right)}\right),
$$

provided that $\delta$ is small enough. Therefore, we can combine (133), (134) and (131) in order to get an estimate for $\left(\delta \dot{V}_{n}, \delta \Psi_{n}\right)$ and $\delta \psi_{n}$. We then consider formula (81) for the increment $\delta V_{n}$, and eventually obtain

$$
\begin{aligned}
&\left\|\left(\delta V_{n}, \delta \Psi_{n}\right)\right\|_{H_{\gamma}^{s}\left(\Omega_{T}\right)}+\left\|\delta \psi_{n}\right\|_{H_{\gamma}^{s+1}\left(\omega_{T}\right)} \\
& \leq C\left\{\left\|f_{n}\right\|_{H_{\gamma}^{s+1}\left(\Omega_{T}\right)}+\left\|h_{n}\right\|_{H_{\gamma}^{s}\left(\Omega_{T}\right)}+\left\|g_{n}\right\|_{H_{\gamma}^{s+1}\left(\omega_{T}\right)}\right. \\
&+\left(\left\|f_{n}\right\|_{H_{\gamma}^{4}\left(\Omega_{T}\right)}+\left\|h_{n}\right\|_{H_{\gamma}^{3}\left(\Omega_{T}\right)}+\left\|g_{n}\right\|_{H_{\gamma}^{4}\left(\omega_{T}\right)}\right) \\
&\left.\left(\delta \theta_{n}^{(s+2-\alpha)_{+}}+\left\|\dot{U}^{a}+V_{n+1 / 2}, \dot{\Phi}^{a}+\Psi_{n+1 / 2}\right\|_{H_{\gamma}^{s+3}\left(\Omega_{T}\right)}\right)\right\},
\end{aligned}
$$

for all integer $s \in[3, \tilde{\alpha}]$. The remaining part of the work is to estimate the right-hand side of (135). Using Lemma 15, (104a) and Proposition 7, (135) becomes

$$
\begin{aligned}
& \left\|\left(\delta V_{n}, \delta \Psi_{n}\right)\right\|_{H_{\gamma}^{s}\left(\Omega_{T}\right)}+\left\|\delta \psi_{n}\right\|_{H_{\gamma}^{s+1}\left(\omega_{T}\right)} \\
& \leq \quad C\left\{\theta_{n}^{s-\alpha-1}\left(\left\|f^{a}\right\|_{H_{\gamma}^{\alpha+1}\left(\Omega_{T}\right)}+\delta^{2}\right)+\delta^{2} \theta_{n}^{L(s+1)-1}\right\} \Delta_{n} \\
& \quad+C \delta \Delta_{n}\left(\theta_{n}^{2-\alpha}\left(\left\|f^{a}\right\|_{H_{\gamma}^{\alpha+1}\left(\Omega_{T}\right)}+\delta^{2}\right)+\delta^{2} \theta_{n}^{9-2 \alpha}\right)\left(\theta_{n}^{(s+3-\alpha)_{+}}+\theta_{n}^{s+4-\alpha}\right) .
\end{aligned}
$$


One checks that, for $\alpha \geq 7$, and $s \in[3, \tilde{\alpha}]$, the following inequalities hold true:

$$
\begin{array}{ll}
L(s+1) \leq s-\alpha, & (s+3-\alpha)_{+}+2-\alpha \leq s-\alpha-1, \\
s+6-2 \alpha \leq s-\alpha-1, & (s+3-\alpha)_{+}+9-2 \alpha \leq s-\alpha-1, \\
s+13-3 \alpha \leq s-\alpha-1 . &
\end{array}
$$

From (136), we thus obtain

$$
\left\|\left(\delta V_{n}, \delta \Psi_{n}\right)\right\|_{H_{\gamma}^{s}\left(\Omega_{T}\right)}+\left\|\delta \psi_{n}\right\|_{H_{\gamma}^{s+1}\left(\omega_{T}\right)} \leq C\left\{\left\|f^{a}\right\|_{H_{\gamma}^{\alpha+1}\left(\Omega_{T}\right)}+\delta^{2}\right\} \theta_{n}^{s-\alpha-1} \Delta_{n},
$$

and (130) follows.

We now check the three remaining inequalities in $\left(H_{n}\right)$.

Lemma 17. - Assume $\alpha \geq 7$. If $\delta>0$ is sufficiently small, $\left\|f^{a}\right\|_{H_{\gamma}^{\alpha+1}\left(\Omega_{T}\right)} / \delta$ is sufficiently small, and $\theta_{0} \geq 1$ is sufficiently large, then for all $3 \leq s \leq \tilde{\alpha}-2$, one has

$$
\left\|\mathcal{L}\left(V_{n}, \Psi_{n}\right)-f^{a}\right\|_{H_{\gamma}^{s}\left(\Omega_{T}\right)} \leq 2 \delta \theta_{n}^{s-\alpha-1} .
$$

Proof. - Recall that, by summing the relations (95), we have

$$
\mathcal{L}\left(V_{n}, \Psi_{n}\right)-f^{a}=\left(S_{\theta_{n-1}}-I\right) f^{a}+\left(I-S_{\theta_{n-1}}\right) E_{n-1}+e_{n-1} .
$$

The proof then follows by applying (78), (126) and (127), provided that $\delta>0$, and $\left\|f^{a}\right\|_{H_{\gamma}^{\alpha+1}\left(\Omega_{T}\right)} / \delta$ are taken sufficiently small.

The following lemma follows exactly with the same arguments:

Lemma 18. - Let $\alpha \geq 7$. If $\delta>0$ is sufficiently small, and $\theta_{0} \geq 1$ is sufficiently large, then for all $4 \leq s \leq \alpha$, one has

$$
\left\|\mathcal{B}\left(\left(V_{n}\right)_{\left.\right|_{x_{2}=0}}, \psi_{n}\right)\right\|_{H_{\gamma}^{s}\left(\omega_{T}\right)} \leq \delta \theta_{n}^{s-\alpha-1} .
$$

Moreover, one has

$$
\left\|\partial_{t} \Psi_{n}+\left(v^{a}+v_{n}\right) \partial_{x_{1}} \Psi_{n}+v_{n} \partial_{x_{1}} \Phi^{a}-u_{n}\right\|_{H_{\gamma}^{3}\left(\Omega_{T}\right)} \leq \delta \theta_{n}^{2-\alpha} .
$$

Lemmas 16, 17 and 18 show that $\left(H_{n-1}\right)$ implies $\left(H_{n}\right)$ provided that $\alpha \geq 7, \tilde{\alpha}=\alpha+4$, (102) holds, $\delta>0$ is small enough, $\left\|f^{a}\right\|_{H_{\gamma}^{\alpha+1}\left(\Omega_{T}\right)} / \delta$ is small enough, and $\theta_{0} \geq 1$ is large enough. We fix $\alpha, \tilde{\alpha}, \delta>0$, and $\theta_{0} \geq 1$ and we finally prove $\left(H_{0}\right)$.

Lemma 19. - If $\left\|f^{a}\right\|_{H_{\gamma}^{\alpha+1}\left(\Omega_{T}\right)} / \delta$ is sufficiently small, then property $\left(H_{0}\right)$ holds.

Proof. - Recall that $V_{0}=\Psi_{0}=\psi_{0}=0$. Thanks to the property of the approximate solution (see Lemma 3), we see that $U^{a}+V_{0}, \Phi^{a}+\Psi_{0}, \varphi^{a}+\psi_{0}$ satisfy the eikonal equations, and the Rankine-Hugoniot jump conditions. Consequently, the contruction of Proposition 7 yields $V_{1 / 2}=\Psi_{1 / 2}=\psi_{1 / 2}=0$. Consider the problem

$$
\begin{array}{ll}
\mathbb{L}_{e}^{\prime}\left(U^{a}, \Phi^{a}\right) \delta \dot{V}_{0}=S_{\theta_{0}} f^{a} & \text { in } \Omega_{T}, \\
\mathbb{B}_{e}^{\prime}\left(U_{\left.\right|_{x_{2}}=0}^{a}, \varphi^{a}\right)\left(\left(\delta \dot{V}_{0}\right)_{\left.\right|_{x_{2}}=0}, \delta \psi_{0}\right)=0 & \text { on } \omega_{T}, \\
\delta \dot{V}_{0}=0, \quad \delta \psi_{0}=0 & \text { for } t<0 .
\end{array}
$$

Since $\left\|\left(\dot{U}^{a}, \dot{\Phi}^{a}\right)\right\|_{H_{\gamma}^{s}\left(\Omega_{T}\right)} \leq \delta$ for all $s \in[3, \tilde{\alpha}+3]$, we may apply (101) and obtain

$$
\left\|\delta \dot{V}_{0}\right\|_{H_{\gamma}^{s}\left(\Omega_{T}\right)}+\left\|\delta \psi_{0}\right\|_{H_{\gamma}^{s+1}\left(\omega_{T}\right)} \leq C\left\|S_{\theta_{0}} f^{a}\right\|_{H_{\gamma}^{s+1}\left(\Omega_{T}\right)} .
$$


Then we find $\delta \Psi_{0}^{ \pm}$from the equations (90), and (91), that read in this case:

$$
\partial_{t} \delta \Psi_{0}^{ \pm}+v^{a \pm} \partial_{x_{1}} \delta \Psi_{0}^{ \pm}+\left\{\partial_{x_{1}} \Phi^{a \pm} \frac{\partial_{x_{2}} v^{a \pm}}{\partial_{x_{2}} \Phi^{a \pm}}-\frac{\partial_{x_{2}} u^{a \pm}}{\partial_{x_{2}} \Phi^{a \pm}}\right\} \delta \Psi_{0}^{ \pm}+\partial_{x_{1}} \Phi^{a \pm} \delta \dot{v}_{0}^{-}-\delta \dot{u}_{0}^{-}=0
$$

Standard energy estimates yield, as in the proof of Lemma 16:

$$
\forall s \in[3, \tilde{\alpha}], \quad\left\|\delta \Psi_{0}\right\|_{H_{\gamma}^{s}\left(\Omega_{T}\right)} \leq C\left\|\delta \dot{V}_{0}\right\|_{H_{\gamma}^{s}\left(\Omega_{T}\right)} .
$$

We finally obtain from (140) and (141):

$$
\left\|\left(\delta V_{0}, \delta \Psi_{0}\right)\right\|_{H_{\gamma}^{s}\left(\Omega_{T}\right)}+\left\|\delta \psi_{0}\right\|_{H_{\gamma}^{s+1}\left(\omega_{T}\right)} \leq C\left\|S_{\theta_{0}} f^{a}\right\|_{H_{\gamma}^{s+1}\left(\Omega_{T}\right)} \leq C \theta_{0}^{(s-\alpha)_{+}}\left\|f^{a}\right\|_{H_{\gamma}^{\alpha+1}\left(\Omega_{T}\right)} .
$$

If $\left\|f^{a}\right\|_{H_{\gamma}^{\alpha+1}\left(\Omega_{T}\right)} / \delta$ is sufficiently small, then

$$
\left\|\left(\delta V_{0}, \delta \Psi_{0}\right)\right\|_{H_{\gamma}^{s}\left(\Omega_{T}\right)}+\left\|\delta \psi_{0}\right\|_{H_{\gamma}^{s+1}\left(\omega_{T}\right)} \leq \delta \theta_{0}^{s-\alpha-1} \Delta_{0}, \quad 3 \leq s \leq \tilde{\alpha} .
$$

The other inequalities in $\left(H_{0}\right)$ are readily satisfied by taking $\left\|f^{a}\right\|_{H_{\gamma}^{\alpha+1}\left(\Omega_{T}\right)}$ small enough. The proof is complete.

From Lemmas 16 - 19, we get that $\left(H_{n}\right)$ holds for every $n \geq 0$, provided that the parameters are well chosen and that the source term $f^{a}$ is small enough.

\section{Conclusion of the proof of Theorem 1}

Given an integer $\alpha \geq 7$, in agreement with the requirements of Lemma 14, we set $\tilde{\alpha}=$ $\alpha+4$. Let $\mu=\alpha-1 \geq 6$. Let us consider initial data $U_{0}^{ \pm}:=\left(\rho_{0}^{ \pm}, v_{0}^{ \pm}, u_{0}^{ \pm}\right)$such that $U_{0}^{ \pm}=\bar{U}^{ \pm}+\dot{U}_{0}^{ \pm}$, where $\dot{U}_{0}^{ \pm} \in H^{\mu+15 / 2}\left(\mathbb{R}_{+}^{2}\right), \varphi_{0} \in H^{\mu+8}(\mathbb{R})$, and that satisfy the compatibility conditions up to order $\mu+7$. Thanks to Lemmas 2 and 3, we may find an approximate solution $\left(U^{a}, \Phi^{a}\right)$ such that $U^{a}=\bar{U}+\dot{U}^{a}$ with $\dot{U}^{a} \in H^{\mu+8}(\Omega), \Phi^{a \pm}= \pm x_{2}+\dot{\Phi}^{a \pm}$, with $\dot{\Phi}^{a} \in H^{\mu+9}(\Omega), \varphi^{a} \in H^{\mu+17 / 2}(\omega)$ and $f^{a} \in H^{\mu+7}(\Omega)$. If $\left\|\dot{U}_{0}^{ \pm}\right\|_{H^{\mu+15 / 2}\left(\mathbb{R}_{+}^{2}\right)}+\left\|\varphi_{0}\right\|_{H^{\mu+8}(\mathbb{R})}$ is sufficiently small, from (69), (74) we obtain (102), and the requirements of Lemmas 16, 17, 18,19 . Hence for small, compatible initial data, the property $\left(H_{n}\right)$ holds true for all $n$. In particular, we have:

$$
\sum_{n \geq 0}\left\|\left(\delta V_{n}, \delta \Psi_{n}\right)\right\|_{H_{\gamma}^{\mu}\left(\Omega_{T}\right)}+\left\|\delta \psi_{n}\right\|_{H_{\gamma}^{\mu+1}\left(\omega_{T}\right)}<+\infty
$$

so the sequences $\left(V_{n}\right)$ and $\left(\Psi_{n}\right)$, converge in $H_{\gamma}^{\mu}\left(\Omega_{T}\right)$ towards some limits $V$ and $\Psi$, and the sequence $\left(\psi_{n}\right)$ converges in $H_{\gamma}^{\mu+1}\left(\omega_{T}\right)$ towards some limit $\psi$. Passing to the limit in (137), (138) for $s=\mu=\alpha-1$, and in (139), we obtain (75). Therefore $U=U^{a}+V, \Phi=\Phi^{a}+\Psi$ is a solution on $\Omega_{T}^{+}$of (3), (4), (5), (6), (7), so the proof of Theorem 1 is complete.

REMARK 8. - The smallness conditions on $\delta$ and $\left\|f^{a}\right\|_{H_{\gamma}^{\alpha+1}\left(\Omega_{T}\right)} / \delta$ are satisfied for suffciently small initial perturbations $\left(\dot{U}_{0}^{ \pm}, \varphi_{0}\right)$ of the piecewise constant vortex sheet (11), see (69) and (74). Notice also that $\left(\dot{U}_{0}^{ \pm}, \varphi_{0}\right)$ should be sufficiently small also for preserving the linearized well-posedness, obtained under the supersonic condition (12), see Theorems 2 and 4. 


\section{Appendix A}

\section{The existence of weakly stable shock waves}

This first appendix is devoted to another application of the approach that we have developed in this paper. More precisely, we still consider the Euler equations (1), but here the space dimension equals 2 or 3 . We still make the assumption $p^{\prime}(\rho)>0$ for all $\rho>0$. Let us denote $d$ the space dimension, and $x=\left(y, x_{d}\right)$ a generic point of the space $\mathbb{R}^{d}, y \in \mathbb{R}^{d-1}$ and $x_{d} \in \mathbb{R}$. We also decompose the velocity $\mathbf{u}$ in (1) as $\mathbf{u}=(v, u), v \in \mathbb{R}^{d-1}$, and $u \in \mathbb{R}$.

We are interested in shock waves solutions to (1). These are smooth solutions on either side of a hypersurface $\Gamma=\left\{x_{d}=\varphi(t, y), t \in[0, T], y \in \mathbb{R}^{d-1}\right\}$, and such that at each time $t \in[0, T]$ and at each point $\left(y, x_{d}\right)$ of the (curve or) surface $\Gamma(t)$, the following conditions are satisfied:

$$
\begin{aligned}
& \rho^{+}\left(u^{+}-v^{+} \cdot \nabla_{y} \varphi-\partial_{t} \varphi\right)=\rho^{-}\left(u^{-}-v^{-} \cdot \nabla_{y} \varphi-\partial_{t} \varphi\right)=: j, \\
& j\left(\mathbf{u}^{+}-\mathbf{u}^{-}\right)+\left(p\left(\rho^{+}\right)-p\left(\rho^{-}\right)\right)\left(\begin{array}{c}
-\nabla_{y} \varphi \\
1
\end{array}\right)=0, \\
& j>0, \quad 0<\frac{u^{+}-v^{+} \cdot \nabla_{y} \varphi-\partial_{t} \varphi}{c\left(\rho^{+}\right) \sqrt{1+\left|\nabla_{y} \varphi\right|^{2}}}<1<\frac{u^{-}-v^{-} \cdot \nabla_{y} \varphi-\partial_{t} \varphi}{c\left(\rho^{-}\right) \sqrt{1+\left|\nabla_{y} \varphi\right|^{2}}} .
\end{aligned}
$$

Observe that the conditions (142a) and (142b) are the Rankine-Hugoniot jump conditions for (1). The condition $j \neq 0$ means that there is a mass transfer from one side of $\Gamma(t)$ to the other, and (142c) are Lax' shock inequalities for a 1-shock wave ${ }^{(3)}$. The existence of shock waves is again a nonlinear free boundary hyperbolic problem: we wish to solve (1) on either side of $\Gamma(t)$, together with the transmission conditions (142a), (142b), and the constraints (142c).

Up to Galilean transformations, the planar shock waves, that is, the piecewise constant solutions of (1), (142) have the form

$$
(\rho, v, u)= \begin{cases}U_{r}:=\left(\rho_{r}, 0, u_{r}\right), & \text { if } x_{d}>0 \\ U_{l}:=\left(\rho_{l}, 0, u_{l}\right), & \text { if } x_{d}<0\end{cases}
$$

where

$$
\rho_{r} u_{r}=\rho_{l} u_{l}=: j, \quad j=\sqrt{\rho_{r} \rho_{l} \frac{p\left(\rho_{r}\right)-p\left(\rho_{l}\right)}{\rho_{r}-\rho_{l}}}, \quad 0<\frac{u_{r}}{c\left(\rho_{r}\right)}<1<\frac{u_{l}}{c\left(\rho_{l}\right)} .
$$

The (linear) stability properties of the planar shock wave (143) are encoded in the following result:

Proposition 8 (Majda [24]). - The shock wave (143) is uniformly stable if, and only if

$$
\frac{u_{r}^{2}}{c\left(\rho_{r}\right)^{2}}\left(\frac{\rho_{r}}{\rho_{l}}-1\right)<1 .
$$

In particular, when $p$ is a convex function of $\rho$, this inequality always holds.

(3) The study of the 3 -shock waves, for which $j<0$, can be carried out in exactly the same way, so we shall not deal with it. However, the results of this appendix extend to the case of 3 -shock waves.

$4^{\mathrm{e}}$ SÉRIE - TOME $41-2008-\mathrm{N}^{\mathrm{o}} 1$ 
In [24, 23], Majda constructs shock waves that are close to a uniformly stable planar shock (see also [31, 29] for a refined version of Majda's result). In other words, Majda solves the equations (1), (142) for initial data that are perturbations of the planar shock (143), and that satisfy the appropriate compatibility conditions. The initial condition for $\varphi$ is a perturbation of 0 since (143) is a stationary shock wave.

When the shock wave (143) satisfies

$$
\frac{u_{r}^{2}}{c\left(\rho_{r}\right)^{2}}\left(\frac{\rho_{r}}{\rho_{l}}-1\right)>1
$$

the planar shock wave (143) is only weakly stable. However, it is proved in [9] that the linearized problem around a variable coefficients small perturbation of the planar shock (143) satisfies an a priori estimate with a loss of one tangential derivative. (This weak stability result is the analogue of Theorem 2 for contact discontinuities.)

Here, we prove the local existence of weakly stable shock waves, which answers, at least for the isentropic Euler equations, the question asked in the introduction of [29].

Under conditions (142), the free boundary is noncharacteristic. Therefore, we do not need to introduce conditions similar to the eikonal equations (7), as we did for contact discontinuities, in order to get a boundary matrix with constant rank in the whole domain. One can straighten the front as in $[24,23,29]$ with the change of variables:

$$
\Phi^{ \pm}\left(t, y, x_{d}\right):=\left(t, y, \pm \kappa x_{d}+\chi\left( \pm x_{d}\right) \varphi(t, y)\right),
$$

where $\chi \in \mathcal{C}_{0}^{\infty}(\mathbb{R})$, equal to 1 on $[-1,1]$, and $\kappa$ is a constant that satisfies $\kappa>2\left\|\chi^{\prime}\right\|_{L^{\infty}(\mathbb{R})}$. The cut-off function is introduced in order to work globally on $\left\{x_{d}>0\right\}$, and we shall consider solutions for which $\|\varphi\|_{L^{\infty}([0, T] \times \mathbb{R})} \leq 1$. The problem can thus be rewritten as the following nonlinear hyperbolic system:

$$
\begin{aligned}
& \partial_{t} U^{ \pm}+\sum_{j=1}^{d-1} A_{j}\left(U^{ \pm}\right) \partial_{x_{j}} U^{ \pm} \\
& \quad+\frac{1}{\partial_{x_{d}} \Phi^{ \pm}}\left(A_{d}\left(U^{ \pm}\right)-\partial_{t} \Phi^{ \pm}-\sum_{j=1}^{d-1} \partial_{x_{j}} \Phi^{ \pm} A_{j}\left(U^{ \pm}\right)\right) \partial_{x_{d}} U^{ \pm}=0
\end{aligned}
$$

in $\left\{x_{d}>0\right\}$, together with the boundary conditions (142) on $\left\{x_{d}=0\right\}$. The matrices $A_{1}, \ldots, A_{d}$ correspond to the quasilinear form of the Euler equations (1) in space dimension $d$, and $U^{ \pm}=\left(\rho^{ \pm}, \mathbf{u}^{ \pm}\right)$. We obtain the following result:

THEOREM 5. - Consider a planar shock wave (143) that satisfies the weak stability condition (144). Let $T>0$, and let $\mu \in \mathbb{N}$ be sufficiently large. Then there exists an integer $\tilde{\mu} \geq \mu$, such that if the initial data $\left(U_{0}^{ \pm}, \varphi_{0}\right)$ have the form

$$
U_{0}^{ \pm}=U_{r, l}+\dot{U}_{0}^{ \pm}
$$

with $\dot{U}_{0}^{ \pm} \in H^{\tilde{\mu}+1 / 2}\left(\mathbb{R}_{+}^{d}\right), \varphi_{0} \in H^{\tilde{\mu}+3 / 2}\left(\mathbb{R}^{d-1}\right)$, if they are compatible up to order $\tilde{\mu}$, have a compact support, and are sufficiently small, then there exists a solution $U^{ \pm}=U_{r, l}+\dot{U}^{ \pm}, \Phi^{ \pm}, \varphi$ to (145), (142), on the time interval $[0, T]$. This solution satisfies $\dot{U}^{ \pm} \in H^{\mu}(] 0, T\left[\times \mathbb{R}^{d-1} \times \mathbb{R}^{+}\right)$, $\varphi \in H^{\mu+1}(] 0, T\left[\times \mathbb{R}^{d-1}\right)$ and $\left(\dot{U}^{ \pm}, \varphi\right)_{\left.\right|_{t=0}}=\left(\dot{U}_{0}^{ \pm}, \varphi_{0}\right)$. 
Sketch of the proof. The proof follows the same steps of that of Theorem 1 for contact discontinuities.

The first step is the weak stability result proved in [9], that is an $L^{2}$ a priori estimate with a loss of one tangential derivative for the solutions of the linearized problem around a variable coefficients small perturbation of the planar shock (143), which satisfies (142). (This result is the analogue of Theorem 2.) Under the assumption (144), the symbol associated with the linearized front is elliptic as for uniformly stable shock waves and contact discontinuities, so one can control the $H_{\gamma}^{1}$ norm of the linearized front. Moreover, the boundary is noncharacteristic, so one can also control the whole trace of the solution to the linearized problem on the boundary. Therefore we have an a priori estimate similar to (27) in Theorem 2, with in the left-hand side the term $\left\|\dot{V}_{\left.\right|_{x_{2}=0}}\right\|_{L_{\gamma}^{2}\left(\mathbb{R}^{2}\right)}$ instead of $\left\|\mathbb{P}(\varphi) \dot{V}_{\left.\right|_{x_{2}=0}}\right\|_{L_{\gamma}^{2}\left(\mathbb{R}^{2}\right)}$, (see [9] for details).

The next step is the solvability of the linearized problem. If we consider the dual problem defined in [29, page 60], then the (backward) weak Lopatinskii condition is satisfied, the zeroes of the Lopatinskii determinant are simple, and they are located in the hyperbolic region of the cotangent of the boundary. This enables us to obtain an energy estimate with a loss of one tangential derivative for the dual problem, which yields a well-posedness result for the linearized equations (this is the analogue of Theorem 3).

At this stage, we can follow the analysis of section 3 and prove a tame estimate in the Sobolev spaces $H_{\gamma}^{m}\left(\Omega_{T}\right)$. The analysis is simpler than what we have done in section 3 because the boundary is noncharacteristic, so all the normal derivatives can be estimated directly through equation (145) by the tangential derivatives, without the use of the vorticity equation (47) as in section 3. We obtain a tame estimate similar to (101), the only difference being the complete control at the boundary, i.e. the term $\left\|\dot{V}_{\left.\right|_{x_{2}=0}}\right\|_{H_{\gamma}^{m}\left(\omega_{T}\right)}$ instead of $\left\|\mathbb{P}(\varphi) \dot{V}_{\left.\right|_{x_{2}=0}}\right\|_{H_{\gamma}^{m}\left(\omega_{T}\right)}$.

To solve the nonlinear problem, it is convenient to proceed as in [29], and to make the boundary conditions linear. This is possible because in (142), the symbol associated with the front $\varphi$ is elliptic. Changing unknowns, we can therefore transform (142a) and (142b) into boundary conditions of the form

$$
\left(\begin{array}{c}
I_{d} \\
0
\end{array}\right) \nabla_{t, y} \varphi+\underline{M} U_{\left.\right|_{x_{d}=0}}=0,
$$

where $\underline{M}$ is a constant matrix of maximal rank. The analysis of the compatibility conditions proceeds as in [29, section 4.2].

The last step is the iteration scheme. Here we use the same Nash-Moser scheme as in the proof of Theorem 1, with the same chain of spaces, and the same family of smoothing operators. The difference is that we need to force only the Rankine-Hugoniot conditions (in their new linear form) during the iteration scheme. This is particularly simple since $\underline{M}$ is of maximal rank, so, up to permuting some columns of $\underline{M}$, we can write

$$
\underline{M}=\left(M_{1} M_{2}\right),
$$

$4^{\mathrm{e}}$ SÉRIE - TOME $41-2008-\mathrm{N}^{\mathrm{o}} 1$ 
where $M_{2}$ is a square invertible matrix. Decomposing the vector $U=(V, W)$ accordingly, we can rewrite the boundary conditions as

$$
-M_{2}^{-1}\left(\begin{array}{c}
I_{d} \\
0
\end{array}\right) \nabla_{t, y} \varphi-M_{2}^{-1} M_{1} V_{\left.\right|_{x_{d}=0}}=W_{\left.\right|_{x_{d}}=0} .
$$

Following the analysis of section 7, we can thus project at each iteration step on the set of functions that satisfy the Rankine-Hugoniot conditions and prove that our modified NashMoser iteration converges towards a solution to the nonlinear equations (145), (142).

\section{Appendix B}

\section{The existence of subsonic phase transitions in a Van der Waals fluid}

In this second appendix, we are interested in a model of isothermal liquid/vapor phase transitions in a van der Waals fluid. We consider the Euler equations (1) in two, or three space dimensions, and we assume that the fluid obeys an isothermal van der Waals pressure law:

$$
p(\rho)=\pi(v):=\frac{R T}{v-b}-\frac{a}{v^{2}}, \quad v:=1 / \rho,
$$

where $R, a$ and $b$ are numerical constants, and $T$ is the fixed temperature of the fluid. When $T$ is below the critical temperature $T_{c}:=8 a /(27 b R)$, the pressure law $p$ is nonmonotone: it is increasing on $] 0, \rho_{1}[\cup] \rho_{2},+\infty[$, and it is decreasing on the interval $] \rho_{1}, \rho_{2}[$. The choice of such a pressure law models the coexistence of liquid $\left(\rho>\rho_{2}\right)$ and vapor phases $\left(\rho<\rho_{1}\right)$, and we are interested in the existence of propagating phase boundaries that connect a liquid, and a vapor state. Recall that the Maxwell points $\left(v_{M}, v_{m}\right)$ are uniquely defined by the relations

$$
v_{M}<v_{m}, \quad \pi\left(v_{m}\right)=\pi\left(v_{M}\right)=\bar{\pi}, \quad \int_{v_{M}}^{v_{m}}(\bar{\pi}-\pi(v)) d v=0
$$

and we denote $\rho_{m, M}=1 / v_{m, M}$, so that $\rho_{m}<\rho_{1}<\rho_{2}<\rho_{M}$. We also denote $e(\rho)$ the free energy per unit volume, that is defined by $\rho e^{\prime}(\rho)-e(\rho)=p(\rho)$.

From now on, we are interested in piecewise smooth solutions of the Euler equations (1), with the pressure law (146), and such that the states on either side of the boundary belong to distinct phases. When the densities on either side of the boundary are close enough to the Maxwell points, it is shown in [4] that Lax' shock inequalities are not satisfied. More precisely, in the terminology of Freistühler, see [15], such propagating phase boundaries are undercompressive shock waves of type 0 . The Rankine-Hugoniot conditions are not sufficient to determine a phase boundary, because there are not enough characteristics impinging the discontinuity. An additional scalar jump condition is required to determine the "admissible" propagating phase boundaries. We refer to [4, 5] and to the references therein for possible admissibility criteria, and we focus here on the so-called capillary criterion that was considered in [4]. We thus want to construct a solution $U$ to the Euler equations (1), that is smooth 
on either side of a hypersurface $\Gamma=\left\{x_{d}=\varphi(t, y)\right\}$, that satisfies the Rankine-Hugoniot jump conditions at each point of $\Gamma$ :

$$
\begin{gathered}
\rho^{+}\left(u^{+}-v^{+} \cdot \nabla_{y} \varphi-\partial_{t} \varphi\right)=\rho^{-}\left(u^{-}-v^{-} \cdot \nabla_{y} \varphi-\partial_{t} \varphi\right)=: j, \\
j\left(\mathbf{u}^{+}-\mathbf{u}^{-}\right)+\left(p\left(\rho^{+}\right)-p\left(\rho^{-}\right)\right)\left(\begin{array}{c}
-\nabla_{y} \varphi \\
1
\end{array}\right)=0, \\
j>0, \quad 0<\frac{u^{ \pm}-v^{ \pm} \cdot \nabla_{y} \varphi-\partial_{t} \varphi}{c\left(\rho^{ \pm}\right) \sqrt{1+\left|\nabla_{y} \varphi\right|^{2}}}<1,
\end{gathered}
$$

together with the generalized equal area rule:

$$
\int_{v^{-}}^{v^{+}} \pi(v) d v=\frac{\pi\left(v^{+}\right)+\pi\left(v^{-}\right)}{2}\left(v^{+}-v^{-}\right) .
$$

Together with (147), the additional jump condition (148) is equivalent to

$$
\left[\frac{1}{2}\left(u-v \cdot \nabla_{y} \varphi-\partial_{t} \varphi\right)^{2}+\left(1+\left|\nabla_{y} \varphi\right|^{2}\right) e^{\prime}\right]=0 .
$$

In (149), we have used the classical notation $[q]:=q^{+}-q^{-}$to denote the jump of a quantity $q$ across the discontinuity. This is again a nonlinear free boundary hyperbolic problem.

Straightening the unknown interface as in Appendix A, we thus want to construct a smooth solution to the system

$$
\begin{aligned}
\partial_{t} U^{ \pm}+\sum_{j=1}^{d-1} A_{j}\left(U^{ \pm}\right) \partial_{x_{j}} U^{ \pm} & \\
& +\frac{1}{\partial_{x_{d}} \Phi^{ \pm}}\left(A_{d}\left(U^{ \pm}\right)-\partial_{t} \Phi^{ \pm}-\sum_{j=1}^{d-1} \partial_{x_{j}} \Phi^{ \pm} A_{j}\left(U^{ \pm}\right)\right) \partial_{x_{d}} U^{ \pm}=0
\end{aligned}
$$

in $\left\{x_{d}>0\right\}$, together with the boundary conditions (147), (149) on $\left\{x_{d}=0\right\}$. Once again, the matrices $A_{1}, \ldots, A_{d}$ correspond to the quasilinear form of the Euler equations (1) in space dimension $d$, and $U^{ \pm}=\left(\rho^{ \pm}, \mathbf{u}^{ \pm}\right)$.

For the sake of completeness, we recall the stability result that was obtained in [4]. Consider a planar phase transition

$$
(\rho, v, u)= \begin{cases}U_{r}:=\left(\rho_{r}, 0, u_{r}\right), & \text { if } x_{d}>0 \\ U_{l}:=\left(\rho_{l}, 0, u_{l}\right), & \text { if } x_{d}<0,\end{cases}
$$

that satisfies $\rho_{r}>\rho_{M}, \rho_{l}<\rho_{m}$, and the jump conditions

$$
\begin{gathered}
\rho_{r} u_{r}=\rho_{l} u_{l}=: j, \quad j=\sqrt{\rho_{r} \rho_{l} \frac{p\left(\rho_{r}\right)-p\left(\rho_{l}\right)}{\rho_{r}-\rho_{l}}}, \quad 0<\frac{u_{r, l}}{c\left(\rho_{r, l}\right)}<1, \\
\int_{v_{r}}^{v_{l}} \pi(v) d v=\frac{p\left(\rho_{r}\right)+p\left(\rho_{l}\right)}{2}\left(v_{r}-v_{l}\right) .
\end{gathered}
$$

We have the following:

Theorem 6 (Benzoni-Gavage [4]). - There exist planar phase transitions (151), with $\rho_{r, l}$ close enough to $\rho_{M, m}$, and these planar phase transitions are weakly stable. In any case, the uniform Lopatinskii condition is not satisfied.

$4^{\mathrm{e}}$ SÉRIE - TOME $41-2008-\mathrm{N}^{\mathrm{o}} 1$ 
The weak stability phenomenon in Theorem 6 is somehow less critical than the weak stability of shock waves in isentropic gas dynamics, since it is due to surface waves (that decay exponentially fast in the normal direction). These surface waves are similar to the well-known Rayleigh waves in elastodynamics.

The symbol associated with the unknown front in (147), (149) is elliptic, and as was done in the preceding section, it is possible to reduce the nonlinear boundary conditions to constant linear boundary conditions. Moreover, this is again a noncharacteristic boundary problem, which allows for a complete control of the solution at the boundary, as in Appendix A.

The basic $L^{2}$ estimate for the linearized equations can be obtained by following exactly the method of [9], and one can also prove the well-posedness of the linearized equations by showing that a dual problem satisfies the backward weak Lopatinskii condition. Consequently, all the analysis is similar to the analysis of weakly stable shock waves in isentropic gas dynamics, and in short, we can positively answer the question asked in [4] and show the existence of isothermal phase transitions:

Theorem 7. - Consider a planar phase transition (151), as given in Theorem 6. Let $T>0$, and let $\mu \in \mathbb{N}$ be sufficiently large. Then there exists an integer $\tilde{\mu} \geq \mu$, such that if the initial data $\left(U_{0}^{ \pm}, \varphi_{0}\right)$ have the form

$$
U_{0}^{ \pm}=U_{r, l}+\dot{U}_{0}^{ \pm}
$$

with $\dot{U}_{0}^{ \pm} \in H^{\tilde{\mu}+1 / 2}\left(\mathbb{R}_{+}^{d}\right), \varphi_{0} \in H^{\tilde{\mu}+3 / 2}\left(\mathbb{R}^{d-1}\right)$, if they are compatible up to order $\tilde{\mu}$, have a compact support, and are sufficiently small, then there exists a solution $U^{ \pm}=$ $U_{r, l}+\dot{U}^{ \pm}, \Phi^{ \pm}, \varphi$ to (150), (147), (149) on the time interval $[0, T]$. This solution satisfies $\dot{U}^{ \pm} \in H^{\mu}(] 0, T\left[\times \mathbb{R}^{d-1} \times \mathbb{R}^{+}\right), \varphi \in H^{\mu+1}(] 0, T\left[\times \mathbb{R}^{d-1}\right)$ and $\left(\dot{U}^{ \pm}, \varphi\right)_{\left.\right|_{t=0}}=\left(\dot{U}_{0}^{ \pm}, \varphi_{0}\right)$.

\section{Appendix C \\ Nonlinear estimates}

In this short appendix, we recall some classical nonlinear tame estimates. The reader is referred to $[2,25,29]$ for the details.

\section{C.1. Nonlinear estimates in weighted spaces}

We recall the notations

$$
\begin{gathered}
\left.\Omega=\mathbb{R}^{2} \times\right] 0,+\infty\left[, \quad \omega=\partial \Omega=\mathbb{R}^{2},\right. \\
\left.\Omega_{T}=\right]-\infty, T[\times \mathbb{R} \times] 0,+\infty\left[, \quad \omega_{T}=\right]-\infty, T[\times \mathbb{R} .
\end{gathered}
$$

We also recall that for all integer $m$ and all $\gamma \geq 1$, the space $H_{\gamma}^{m}\left(\Omega_{T}\right)=\exp (\gamma t) H^{m}\left(\Omega_{T}\right)$ is equipped with the norm

$$
\|u\|_{H_{\gamma}^{m}\left(\Omega_{T}\right)}:=\left\|\mathrm{e}^{-\gamma t} u\right\|_{H^{m}\left(\Omega_{T}\right)} \approx \sum_{|\alpha| \leq m} \gamma^{m-|\alpha|}\left\|\mathrm{e}^{-\gamma t} \partial^{\alpha} u\right\|_{L^{2}\left(\Omega_{T}\right)},
$$

where the $\approx$ sign denotes equivalent norms, and the constant in the equivalence is independent of $\gamma \geq 1$ and $T>0$ (see [29, page 80]). The definition of the space $H_{\gamma}^{m}\left(\omega_{T}\right)$ is similar.

For all real number $p \geq 1$, the space $L_{\gamma}^{p}\left(\Omega_{T}\right)$ denotes the set of measurable functions such that $\mathrm{e}^{-2 \gamma t / p} u \in L^{p}\left(\Omega_{T}\right)$. The norm is defined in an obvious way. 
With these notations, the Gagliardo-Nirenberg estimates in $H_{\gamma}^{m}\left(\Omega_{T}\right)$ read as follows:

TheOREM 8 (Gagliardo-Nirenberg). - Let $m \geq 1$ be an integer, let $\gamma \geq 1$, and let $T \in \mathbb{R}$. There exists a constant $C$ (that is independent of $\gamma$ and $T$ ) such that for all $u \in H_{\gamma}^{m}\left(\Omega_{T}\right) \cap$ $L^{\infty}\left(\Omega_{T}\right)$, for all multi-integer $\alpha \in \mathbb{N}^{3}$ with $|\alpha| \leq m$, one has

$$
\left\|\partial^{\alpha} u\right\|_{L_{\gamma}^{2 p}\left(\Omega_{T}\right)} \leq C\|u\|_{L^{\infty}\left(\Omega_{T}\right)}^{1-1 / p}\|u\|_{H_{\gamma}^{m}\left(\Omega_{T}\right)}^{1 / p}, \quad \frac{1}{p}=\frac{|\alpha|}{m} .
$$

There is a similar result with $\omega_{T}$ instead of $\Omega_{T}$. (The constant is still independent of $\gamma$ and $T$.)

This result can be used to prove the following tame estimates for products of functions in $H_{\gamma}^{m}\left(\Omega_{T}\right)$ :

Theorem 9. - Let $m \geq 1$ be an integer, let $\gamma \geq 1$, and let $T \in \mathbb{R}$. For all functions $u, v \in H_{\gamma}^{m}\left(\Omega_{T}\right) \cap L^{\infty}\left(\Omega_{T}\right)$, the product uv belongs to $H_{\gamma}^{m}\left(\Omega_{T}\right)$ and satisfies the estimate

$$
\|u v\|_{H_{\gamma}^{m}\left(\Omega_{T}\right)} \leq C\left(\|u\|_{L^{\infty}\left(\Omega_{T}\right)}\|v\|_{H_{\gamma}^{m}\left(\Omega_{T}\right)}+\|u\|_{H_{\gamma}^{m}\left(\Omega_{T}\right)}\|v\|_{L^{\infty}\left(\Omega_{T}\right)}\right) .
$$

Moreover, for all multi-integers $\alpha, \beta$, such that $|\alpha|+|\beta| \leq m$, one has

$$
\left\|\partial^{\alpha} u \partial^{\beta} v\right\|_{L_{\gamma}^{2}\left(\Omega_{T}\right)} \leq C\left(\|u\|_{L^{\infty}\left(\Omega_{T}\right)}\|v\|_{H_{\gamma}^{m}\left(\Omega_{T}\right)}+\|u\|_{H_{\gamma}^{m}\left(\Omega_{T}\right)}\|v\|_{L^{\infty}\left(\Omega_{T}\right)}\right) .
$$

The constant $C$ only depends on $m$, and is independent of $\gamma$ and $T$. The same result holds with $\omega_{T}$ instead of $\Omega_{T}$.

There is also a tame estimate for composed functions:

Theorem 10. - Let $m \geq 1$ be an integer, let $\gamma \geq 1$, and let $T \in \mathbb{R}$. Let $F$ denote a $\mathcal{C}^{\infty}$ function, that is defined on $\mathbb{R}^{q}$ (or on a neighborhood of the origin in $\mathbb{R}^{q}$ ), and that satisfies $F(0)=0$. Then for all $u \in H_{\gamma}^{m}\left(\Omega_{T}\right) \cap L^{\infty}\left(\Omega_{T}\right)$, the composed function $F(u)$ belongs to $H_{\gamma}^{m}\left(\Omega_{T}\right)$ and satisfies the estimate

$$
\|F(u)\|_{H_{\gamma}^{m}\left(\Omega_{T}\right)} \leq C\left(\|u\|_{L^{\infty}\left(\Omega_{T}\right)}\right)\|u\|_{H_{\gamma}^{m}\left(\Omega_{T}\right)},
$$

where $C$ is an increasing function that does not depend on $\gamma$ and $T$.

In the paper we also use the following inequalities in $H_{\gamma}^{m}$ :

THeOREM 11. - The following inequalities hold, with a constant $C$ that is independent of $\gamma \geq 1$ :

$$
\begin{aligned}
& \sqrt{\gamma}\left\|\mathrm{e}^{-\gamma t} V\right\|_{L^{\infty}\left(\Omega_{T}\right)} \leq C\|V\|_{H_{\gamma}^{2}\left(\Omega_{T}\right)},\left\|\mathrm{e}^{-\gamma t} V\right\|_{W^{1, \infty}\left(\Omega_{T}\right)} \leq C \sqrt{\gamma}\|V\|_{H_{\gamma}^{3}\left(\Omega_{T}\right)}, \\
& \left\|\mathrm{e}^{-\gamma t} \psi\right\|_{L^{\infty}\left(\omega_{T}\right)} \leq \frac{C}{\gamma}\|\psi\|_{H_{\gamma}^{2}\left(\omega_{T}\right)}, \quad\left\|\mathrm{e}^{-\gamma t} \psi\right\|_{W^{1, \infty}\left(\omega_{T}\right)} \leq C\|\psi\|_{H_{\gamma}^{3}\left(\omega_{T}\right)} .
\end{aligned}
$$




\section{C.2. Nonlinear functions}

Following [29, page 81], we introduce the following definition:

Definition 2. - Let $k \in \mathbb{N}$. A nonlinear function of order $\leq k$ is a finite sum of the form:

$$
\mathcal{F}(u)=F_{0}(u)+\sum_{\ell=1}^{k} \sum_{\alpha_{1}+\cdots+\alpha_{\ell} \leq k} F_{\ell, \alpha_{1}, \ldots, \alpha_{\ell}}(u)\left\{\nabla^{\alpha_{1}} u, \ldots, \nabla^{\alpha_{\ell}} u\right\},
$$

where $F_{0} \in \mathcal{C}^{\infty}$ and $F_{0}(0)=0$, and where the $\ell$-multilinear mappings $F_{\ell, \alpha_{1}, \ldots, \alpha_{\ell}}(u)$ are $\mathcal{C}^{\infty}$ functions of $u$.

We have the following generalization of Theorem 10:

Theorem 12. - Let $k \in \mathbb{N}, s \in \mathbb{R}$ with $k \leq s$, and let $\mathcal{F}$ be a nonlinear function of order $\leq k$. Then for all $u \in H^{s}\left(\mathbb{R}_{+}^{2}\right) \cap L^{\infty}\left(\mathbb{R}_{+}^{2}\right)$, the composed function $\mathcal{F}(u)$ belongs to $H^{s-k}\left(\mathbb{R}_{+}^{2}\right)$ and satisfies the estimate

$$
\|\mathcal{F}(u)\|_{H^{s-k}\left(\mathbb{R}_{+}^{2}\right)} \leq C\left(\|u\|_{L^{\infty}\left(\mathbb{R}_{+}^{2}\right)}\right)\|u\|_{H^{s}\left(\mathbb{R}_{+}^{2}\right)},
$$

where $C$ is an increasing function.

C.2.0.1. Acknowledgments. - Research of the authors was supported by the EU financed network HYKE, HPRN-CT-2002-00282. The first author would like to warmly thank Sylvie Benzoni-Gavage for introducing him to the subject and for her encouragement during this project.

\section{REFERENCES}

[1] S. Alinhac, Existence d'ondes de raréfaction pour des systèmes quasi-linéaires hyperboliques multidimensionnels, Comm. Partial Differential Equations 14 (1989), 173230.

[2] S. Alinhac, S. GÉrard, Opérateurs pseudo-différentiels et théorème de Nash-Moser, InterÉditions, 1991.

[3] M. Artola, A. Majda, Nonlinear development of instabilities in supersonic vortex sheets. I. The basic kink modes, Phys. D 28 (1987), 253-281.

[4] S. Benzoni-Gavage, Stability of multi-dimensional phase transitions in a van der Waals fluid, Nonlinear Anal. 31 (1998), 243-263.

[5] S. Benzoni-Gavage, Stability of subsonic planar phase boundaries in a van der Waals fluid, Arch. Ration. Mech. Anal. 150 (1999), 23-55.

[6] A. Blokhin, Y. Trakninin, Stability of strong discontinuities in fluids and MHD, in Handbook of mathematical fluid dynamics, Vol. I, North-Holland, 2002, 545-652.

[7] J. Chazarain, A. Piriou, Introduction to the theory of linear partial differential equations, Studies in Mathematics and its Applications 14, North-Holland Publishing Co., 1982.

[8] J.-Y. Chemin, Dynamique des gaz à masse totale finie, Asymptotic Anal. 3 (1990), 215220. 
[9] J.-F. Coulombel, Weakly stable multidimensional shocks, Ann. Inst. H. Poincaré Anal. Non Linéaire 21 (2004), 401-443.

[10] J.-F. Coulombel, Well-posedness of hyperbolic initial boundary value problems, $J$. Math. Pures Appl. 84 (2005), 786-818.

[11] J.-F. Coulombel, A. Morando, Stability of contact discontinuities for the nonisentropic Euler equations, Ann. Univ. Ferrara Sez. VII (N.S.) 50 (2004), 79-90.

[12] J.-F. Coulombel, P. Secchi, The stability of compressible vortex sheets in two space dimensions, Indiana Univ. Math. J. 53 (2004), 941-1012.

[13] J. A. Fejer, J. W. Miles, On the stability of a plane vortex sheet with respect to threedimensional disturbances, J. Fluid Mech. 15 (1963), 335-336.

[14] J. Francheteau, G. Métivier, Existence de chocs faibles pour des systèmes quasilinéaires hyperboliques multidimensionnels, Astérisque 268 (2000), 198.

[15] H. FreistüHLER, Some results on the stability of non-classical shock waves, J. Partial Differential Equations 11 (1998), 25-38.

[16] O. GuÈs, Problème mixte hyperbolique quasi-linéaire caractéristique, Comm. Partial Differential Equations 15 (1990), 595-645.

[17] R. S. Hamilton, The inverse function theorem of Nash and Moser, Bull. Amer. Math. Soc. (N.S.) 7 (1982), 65-222.

[18] E. Harabetian, A convergent series expansion for hyperbolic systems of conservation laws, Trans. Amer. Math. Soc. 294 (1986), 383-424.

[19] L. Hörmander, Implicit function theorems, Stanford university lecture notes, 1977.

[20] T. Kaто, The Cauchy problem for quasi-linear symmetric hyperbolic systems, Arch. Rational Mech. Anal. 58 (1975), 181-205.

[21] P. D. Lax, Hyperbolic systems of conservation laws. II, Comm. Pure Appl. Math. 10 (1957), 537-566.

[22] J.-L. Lions, E. Magenes, Problèmes aux limites non homogènes et applications. Vol. 2, Travaux et Recherches Mathématiques, No. 18, Dunod, 1968.

[23] A. Majda, The existence of multidimensional shock fronts, Mem. Amer. Math. Soc. 43 (1983), 93.

[24] A. Majda, The stability of multidimensional shock fronts, Mem. Amer. Math. Soc. 41 (1983), 95.

[25] A. MAJDA, Compressible fluid flow and systems of conservation laws in several space variables, Applied Mathematical Sciences 53, Springer, 1984.

[26] A. Majda, S. Osher, Initial-boundary value problems for hyperbolic equations with uniformly characteristic boundary, Comm. Pure Appl. Math. 28 (1975), 607-675.

[27] G. Métivier, Interaction de deux chocs pour un système de deux lois de conservation, en dimension deux d'espace, Trans. Amer. Math. Soc. 296 (1986), 431-479.

[28] G. Métivier, Ondes soniques, J. Math. Pures Appl. 70 (1991), 197-268.

[29] G. MÉTIVIER, Stability of multidimensional shocks, in Advances in the theory of shock waves, Progr. Nonlinear Differential Equations Appl. 47, Birkhäuser, 2001, 25-103.

[30] J. W. Miles, On the disturbed motion of a plane vortex sheet, J. Fluid Mech. 4 (1958), 538-552.

$4^{\mathrm{e}}$ SÉRIE - TOME $41-2008$ - No 1 
[31] A. Mokrane, Problèmes mixtes hyperboliques non-linéaires, Ph.D. Thesis, Université de Rennes I, 1987.

[32] A. Morando, P. Trebeschi, Stability of contact discontinuities for the nonisentropic Euler equations in two-space dimensions, preprint, 2007.

[33] J. B. Rauch, F. J. I. Massey, Differentiability of solutions to hyperbolic initialboundary value problems, Trans. Amer. Math. Soc. 189 (1974), 303-318.

[34] S. Schochet, The compressible Euler equations in a bounded domain: existence of solutions and the incompressible limit, Comm. Math. Phys. 104 (1986), 49-75.

[35] P. SeCCHI, The initial-boundary value problem for linear symmetric hyperbolic systems with characteristic boundary of constant multiplicity, Differential Integral Equations 9 (1996), 671-700.

[36] P. Secchi, Well-posedness of characteristic symmetric hyperbolic systems, Arch. Rational Mech. Anal. 134 (1996), 155-197.

[37] D. Serre, Systems of conservation laws. 2, Cambridge University Press, 2000, Geometric structures, oscillations, and initial-boundary value problems.

[38] T. C. Sideris, Formation of singularities in three-dimensional compressible fluids, Comm. Math. Phys. 101 (1985), 475-485.

[39] H. Beirão da Veiga, On the barotropic motion of compressible perfect fluids, Ann. Scuola Norm. Sup. Pisa Cl. Sci. 8 (1981), 317-351.

(Manuscrit reçu le 31 janvier 2007; accepté, après révision, le 26 novembre 2007.)

\author{
Jean-François Coulombel \\ CNRS, Team Project SIMPAF of INRIA Futurs, et \\ Université Lille 1 \\ Laboratoire Paul Painlevé \\ Cité Scientifique \\ 59655 Villeneuve d'Ascq Cedex, France \\ E-mail: jfcoulom@math.univ-lille1.fr \\ Paolo SECCHI \\ Dipartimento di Matematica \\ Facoltà di Ingegneria \\ Via Valotti, 9 \\ 25133 Brescia, Italy \\ E-mail: paolo.secchi@ing.unibs.it
}

